\title{
New anti-epileptic drugs in pharmacoresistant epilepsy : retention time as outcome parameter
}

Citation for published version (APA):

Bootsma, H. P. (2009). New anti-epileptic drugs in pharmacoresistant epilepsy : retention time as outcome parameter. [Doctoral Thesis, Maastricht University]. Datawyse / Universitaire Pers Maastricht. https://doi.org/10.26481/dis.20090604hb

Document status and date:

Published: 01/01/2009

DOI:

10.26481/dis.20090604hb

Document Version:

Publisher's PDF, also known as Version of record

\section{Please check the document version of this publication:}

- A submitted manuscript is the version of the article upon submission and before peer-review. There can be important differences between the submitted version and the official published version of record.

People interested in the research are advised to contact the author for the final version of the publication, or visit the DOI to the publisher's website.

- The final author version and the galley proof are versions of the publication after peer review.

- The final published version features the final layout of the paper including the volume, issue and page numbers.

Link to publication

\footnotetext{
General rights rights.

- You may freely distribute the URL identifying the publication in the public portal. please follow below link for the End User Agreement:

www.umlib.nl/taverne-license

Take down policy

If you believe that this document breaches copyright please contact us at:

repository@maastrichtuniversity.nl

providing details and we will investigate your claim.
}

Copyright and moral rights for the publications made accessible in the public portal are retained by the authors and/or other copyright owners and it is a condition of accessing publications that users recognise and abide by the legal requirements associated with these

- Users may download and print one copy of any publication from the public portal for the purpose of private study or research.

- You may not further distribute the material or use it for any profit-making activity or commercial gain

If the publication is distributed under the terms of Article $25 \mathrm{fa}$ of the Dutch Copyright Act, indicated by the "Taverne" license above, 
New anti-epileptic drugs in
pharmacoresistant epilepsy: retention time as outcome parameter 
(C) H.P.R. Bootsma, Maastricht 2009

ISBN: 9789052788296

Universitaire Pers Maastricht

Layout, cover design \& print: Datawyse bv 


\section{New anti-epileptic drugs in pharmacoresistant epilepsy: retention time as outcome parameter}

\section{PROEFSCHRIFT}

ter verkrijging van de graad van doctor aan de Universiteit Maastricht, op gezag van de Rector Magnificus, Prof. mr. G.P.M.F. Mols volgens het besluit van het College van Decanen, in het openbaar te verdedigen op donderdag 4 juni 2009 om 14.00 uur

door

\section{Hans Peter Ronald Bootsma}

geboren 14 maart 1960 te Hoogeveen

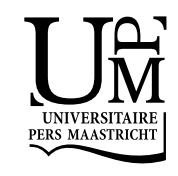




\section{Promotores}

Prof. dr. A.P. Aldenkamp

Prof. dr. Y.A. Hekster, Radboud Universiteit Nijmegen

\section{Copromotor}

Dr. ir. J.A.R.J. Hulsman, Epilepsiecentrum Kempenhaeghe

\section{Beoordelingscommissie}

Prof. dr. M. Limburg (voorzitter)

Prof. dr. P.A.J.M. Boon, Universiteit van Gent, België

Prof. dr. R.P. Koopmans

Dr. P.D. Knoester, Ziekenhuisapotheek Rijnland ziekenhuis, Leiderdorp

Prof. dr. C. Neef

Sponsoring for publication of this thesis was kindly provided by:

Department of Research and Development of the Epilepsy Centre Kempenhaeghe

St Anna Zorggroep te Geldrop, Stichting KNMP-fondsen te Den Haag, GlaxoSmithKline te Zeist, Janssen-Cilag te Tilburg, Novartis te Arnhem, Pfizer te Capelle a/d IJssel, Roche te Woerden, Sanofi-Aventis te Gouda, UCB te Breda. 


\section{Contents}

$\begin{array}{ll}\text { one Introduction } & 7\end{array}$

$\begin{array}{ll}\text { two Clinical evaluation of anti-epileptic drugs. } & 13\end{array}$

Why do we need more than regulatory trials?

three Topiramate in clinical practice: long-term experience in patients 31 with refractory epilepsy referred to a tertiary epilepsy center.

four Levetiracetam in clinical practice: long-term experience in patients with refractory epilepsy referred to a tertiary epilepsy center.

five Lamotrigine in clinical practice: long-term experience in patients with refractory epilepsy referred to a tertiary epilepsy center.

six Long-term effects of levetiracetam and topiramate in clinical practice: a head-to-head comparison.

seven The effect of anti-epileptic drugs on cognition: patient perceived cognitive problems of topiramate versus levetiracetam in clinical practice.

eight Ketter's hypothesis of the mood effects of anti-epileptic drugs coupled 105 to the mechanism of action of topiramate and levetiracetam.

nine The impact of side effects on long-term retention in three new antiepileptic drugs.

ten General discussion

Summary

Samenvatting

eleven Dankwoord

Curriculum vitae

List of publications 



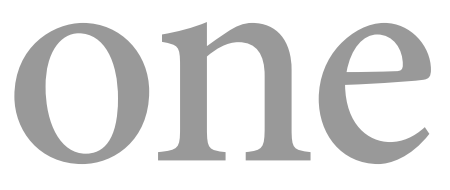

Introduction 
Epilepsy is a neurological disorder characterised by recurrent unprovoked seizures. Epilepsy is not a uniform condition, but comprises many different seizure types and epilepsy syndromes based on heterogeneous etiologies ${ }^{1}$. An epileptic seizure is the clinical manifestation of epilepsy. These clinical manifestations are sudden and transient and can include a wide variety of movement, feeling or psychic disturbances, with or without alteration in consciousness. Seizures are broadly divided into two categories: partial and generalised. Partial seizures arise in an epileptogenic region in one hemisphere of the cerebral cortex. They are subdivided into simple partial seizures, which occur without alteration of consciousness, and complex partial seizures in which consciousness is impaired or lost. These seizures may generalise into a secondary generalised tonic clonic seizure. Primary generalised seizures are characterised by more diffuse neuronal discharges involving both hemispheres of the brain at once and always result in loss of consciousness.

The point prevalence of epilepsy is around $0.5-1 \%$ and it is assumed that there are about 100,000 persons with epilepsy in the Netherlands. The incidence rate of epilepsy is age related. The highest incidence rate (ranging from 100 to 233 per $100,000)$ is observed in children younger than 1 year. Subsequently, this declines in early childhood to around 60 per 100,000. The incidence rate plateaus in adolescents and adults to around 30-40 per 100,000 and rises again in elderly people to 100-170 per 100,000 after the age of 65 years $^{2}$. Epilepsy and side effects of epilepsy treatment have a great impact on patients' lives ${ }^{3}$. The evaluation of side effects should receive more attention in daily practice ${ }^{4}$.

\section{TREATMENT OF EPILEPS Y}

Drug therapy is the mainstay of epilepsy treatment. In very severe treatment resistant cases, some patients are candidates for brain surgery (in the Netherlands approximately 50/year). For other patients implantation of a nervus vagus stimulator is a treatment option. Finally patients can be treated with a ketogenic diet.

However, almost every patient with epilepsy is treated with anti-epileptic drugs (AEDs). The aim of drug treatment is to prevent the occurrence of seizures completely. Approximately $20-30 \%$ of the patients do not become seizure free on anti-epileptic drugs. In addition, many patients suffer from side effects. Therefore it is important to find new anti-epileptic drugs and to evaluate them adequately, i.e. with outcomes that clinicians recognize as clinically relevant. If seizure freedom cannot be achieved than the aim of drug therapy should be a good balance between seizure control and optimal quality of life. 
For a good interpretation of the results presented in this thesis it is important to realise that the likelihood to respond to drug therapy is probably a function of the past treatment history. Drug resistance seems to be a graded process that follows a mono-exponential course with a half-decay constant of 1.5-2 antiepileptic drugs. Thus for every two AEDs that proved ineffective in the past, the percentage of patients who responded with a greater than $50 \%$ reduction in seizure frequency decreases by twofold 5 .

\section{ORGANISATION OF CARE FOR EPILEPSY PATIENTS}

In the Netherlands newly diagnosed epilepsy patients are treated by their neurologists in general hospitals. Patients can be referred to an epilepsy center if their epilepsy does not respond well to drug therapy. These patients suffer from seizures despite drug therapy. Their epilepsy is called refractory. In the Netherlands there are two specialised epilepsy centers. The studies in this thesis were carried out in Epilepsy Center Kempenhaeghe (www.kempenhaeghe.nl). In Kempenhaeghe clinical production/year for epilepsy patients is 2000 admissions, 33,000 hospital days (short stay), 106,000 hospital days (long stay) and 12,000 outpatient visits ${ }^{6}$.

\section{DEVELOPMENT OF NEW ANTI-EPILEPTIC DRUGS}

The European Medicines Agency (EMEA) offers a note for guidance on clinical investigation of medicinal products in the treatment of epileptic disorders ${ }^{7}$. A new draft guideline on this topic is expected to be released for consultation in the first quarter of 2009. In chapter 2 we discuss the outcome measures which are used in regulatory trials and compare them to guidelines from the FDA and ILAE.

Before their market approval new anti-epileptic drugs are tested in patients with refractory epilepsies. The new anti-epileptic drug or a placebo is added to their medication and the patients are followed for 3-6 months. Patients are considered responders if they experience a $50 \%$ seizure reduction or more during a predefined period within this 3-6 months trial duration. If the new anti-epileptic drug is more effective than placebo, and the drug has a good tolerability, it gains market approval. However, market approval is not similar to clinical approval. This requires evaluation of much longer periods. In fact 'seizure free' would imply in some trials that a patient did not have any seizures during the last month of a 3 months trial.

During the last years, a number of new anti-epileptic drugs were introduced in clinical practice in the Netherlands. Some were withdrawn because of idiosyncratic side-effects (such as vigabatrin or felbamate), some because of disappointing efficacy 
(such as tiagabine) others because of potentially better effects in other indications such as gabapentin. The most important new drugs that have a stable position in clinical practice were lamotrigine (introduced in 1996), topiramate (introduced in 1999) and levetiracetam (introduced in 2001). These new anti-epileptic drugs were considered effective and safe after the regulatory trials but no comparative information was available The drugs were not directly compared in a prospective trial. Also safety data were scarce. Before market introduction of levetiracetam, for example, only 5000 patients were treated with the drug. Finally only data for limited timeperiods were available (months, whereas patients with epilepsy are treated for years).

\section{STRUCTURE OF THE THESIS}

In order to assess the value of three important new anti-epileptic drugs in daily practice we decided to evaluate the first patients who were treated with lamotrigine, topiramate or levetiracetam by neurologists in a tertiary referral centre (epilepsy center Kempenhaeghe). Each patient group consisted of 300-400 patients. We collected data from our electronic medical information system (MIS) and from individual patient records. If necessary, treating neurologists were asked for additional information. We chose retention rate as our primary outcome measure. In chapter 2 we outline why the retention rate is a useful composite parameter which reflects the combined effect of efficacy and tolerabity of a new anti-epileptic drug. The retention time is the time that an individual patient continues to use a new drug when in fact he or she needs treatment. The retention rate is the number of patients who still continue taking the drug after a given time interval divided by the number of patients that started with this drug. It is assumed that the retention rate reflects the combination of efficacy and tolerability in the real world ${ }^{8}$.

We think that a patient continues to take a drug if it is effective and tolerated well. Therefore this is considered to be the most clinical relevant measure in patients with chronic refractory epilepsy.

While studying retention time and retention rate additional parameters were collected: reasons for continuation or discontinuation of the drug, the role of efficacy and tolerability in drug withdrawal, the occurrence (and possible disappearance) of side-effects over time, the persistence of efficacy over time or indications for the development of tolerance. 
We aimed at developing clinical useful information on the newer anti-epileptic drugs in the population of patients with refractory epilepsy. We used retention rate as the clinical relevant outcome parameter. Our electronic patient records and chart system allowed us to study all aspects around retention rate in detail. This provided much more information than simple calculation of the retention rate: "The devil is in the details". Not all newer anti-epileptic drugs were included in our studies: only those drugs that were "here to stay" lamotrigine, topiramate and levetiracetam.

OUTLINE OF THE THESIS

This thesis follows the subsequent analysis of the different drugs and in two chapters shows comparative data. The introductional chapter 2 evaluates the outcome measures that are generally used in anti-epileptic drug trials.

In chapter 3 we describe our experience with topiramate. In chapter 4 the results of our levetiracetam study are reported. In chapter 5 efficacy and safety of lamotrigine is evaluated. Plasma levels were monitored for this drug and related to the use of enzyme-inducing anti-epileptic drugs and valproate. Chapter 6 compares the efficacy and the side effect profiles of topiramate and levetiracetam. Chapter 7 focuses on the cognitive side effects of topiramate versus the cognitive side effects of levetiracetam which we found in clinical practice. In chapter 8 the effect on mood of topiramate is compared with the mood-modulating profile of levetiracetam. In chapter 9 the impact of side effects on long-term retention of topiramate, levetiracetam and lamotrigine is presented. 


\section{R E FER E NCES}

1. Knoester PD. Assessment of the value of lamotrigine in daily practice. $\mathrm{PhD}$ thesis printed by van Gogh, Zwijndrecht, The Netherlands 2005.

2. Panayiotopoulos CP. A clinical guide to epileptic syndromes and their treatment. Second edition. Springer-Verlag London Ltd 2007.

3. Auriel E, Landov H, Blatt I, Gandelman-Marton R, Chistik V, Margolin N et al. Quality of life in seizure-free patients with epilepsy on monotherapy. Epilepsy Behav. 2008; 31 oct (Epub ahead of print).

4. Toledano R and Gil-Nagel A. Adverse effects of antiepileptic drugs. Semin Neurol. 2008;28(3):317-27

5. Schiller Y and Najjar Y. Quantifying the response to antiepileptic drugs. Effect of past treatment history. Neurology 2008;70:54-65.

6. Kempenhaeghe, jaarverslag 2006.

7. The European Agency for the Evaluation of Medicinal Products. Note for guidance on clinical investigation of medicinal products in the treatment of epileptic disorders. London, 16 november 2000 CPMP/EWP/566/98 rev 1.

8. Sander JW. New antiepileptic drugs in practice. How do they perform in the real world? Acta Neurol Scand Suppl. 2005;181:26-9. 


\section{Clinical evaluation of anti-epileptic drugs. Why do we need more than regulatory trials?}

H.P.R. Bootsma, L. Jacobs, A.P. Aldenkamp and Y.A. Hekster 


\section{A B S T R A C T}

Study objectives: To describe the pros and cons of regulatory clinical trials for our current knowledge with respect to the use of anti-epileptic drugs.

Method: A systematic search of the literature on clinical trial designs and outcome measures for the evaluation of anti-epileptic drugs.

Results: Regulatory authorities have made guidelines for the measurement of drug efficacy in anti-epileptic drug trials, however the current process of drug trials do not always provide clinical relevant information.

Discussion: The antiepileptic drug approval process is based primarily on short-term, randomised, placebo-controlled, parallel-group, add-on studies. These studies find anticonvulsive molecules which are more effective than placebo but cannot tell us which new anti-epileptic drug will be best in clinical practice. Long-term studies can give valuable information on effectiveness in clinical practice. 


\section{N T ROD UCT I O N}

Drug development must satisfy different goals ${ }^{1}$. The goal of the pharmaceutical industry is to obtain drug approval from regulatory authorities. The goal of regulatory authorities is to obtain reliable evidence that a new anti-epileptic drug (AED) is approvable based upon efficacy and safety criteria. The goal of physicians is to obtain information that enables them to select the best treatment for their individual patients. Is the new AED an improvement to existing drugs or not? Physicians also want to know how to use a new AED in order to get a good balance between effectiveness and side effects. In this article we describe the requirements for regulatory trials on AEDs. The guidelines of the EMEA, the ILAE and the FDA are described.The questions which are left unanswered by regulatory trials are discussed. Limitations of regulatory trials can be divided in three categories: selected patient population, short duration of the trial and differences between treatment according to the trial protocol versus treatment in clinical practice. We review other trial designs and other outcome measures that may help physicians to select the best treatment for their individual patients.

We studied regulatory guidelines of the EMEA, ILAE and the FDA and performed a systematic search on the relevant literature on the topic of outcome measures in evaluating efficacy of AEDs. We searched three databases (PubMed, Medline en Cochrane Library). We did not restrict the timeframe of selected literature.

\section{Regulatory authorities and guidelines for anti-epileptic drug trials}

Authorities for registration such as The European Agency for the Evaluation of Medicinal Products (EMEA), have developed a note of guidance in which advice is being given on the use of different measures of efficacy ${ }^{2}$. Also primary outcomes, which are recommended by the ILAE and the FDA, are discussed.

\section{Primary outcomes as described by the EMEA, ILAE and the FDA}

\section{European Agency for the Evaluation of Medicinal Products (EMEA)}

According to the EMEA the initial evaluation process for a new AED involves a determination of its efficacy in reducing the frequency of seizures in patients who continue to have them despite therapy with an adequate dosage of appropriate drug(s). Usually, partial epilepsies represent the first target, since they are the most frequent, and a substantial percentage of them are not well controlled. In these add-on trials a new $\mathrm{AED} /$ or placebo is added to the 1 or 2 pre-existing AEDs of the the patient. Whenever possible, the dose of the pre-existing drugs is kept stable. 
Once the efficacy of the new AED in combination with other AEDs has been determined, it is important to assess the efficacy of the product when given as monotherapy. The monotherapy studies are generally conducted in the following settings: conversion to monotherapy in patients with multiple-drug treatment, monotherapy trials in newly diagnosed patients, monotherapy in patients undergoing presurgical evaluation for refractory partial epilepsy. The monotherapy indication is usually granted when the efficacy and safety of the new AED has been proven in newly diagnosed patients.

The EMEA states that the assessment of efficacy should be based primarily upon seizure frequency. The period during which seizures are counted has to be known prior to the start of the trial. In doing so the chance of introducing bias is minimised. In order to facilitate accurate analysis of seizure data one has to dichotomise the data into responders and non-responders. Categories can be made using an arbitrary seizure reduction of at least $50 \%^{2}$. In monotherapy studies measures of efficacy are primarily based on the group of patients that is being studied. In newly diagnosed patients, the primary efficacy variable should be based on the proportion of patients remaining seizure free for at least six months. In conversion of add-on to monotherapy, a treatment retention time may be an acceptable endpoint.

In addition to these so-called primary endpoints, secondary endpoints can be used in studying an AED. Secondary endpoints can be: the distribution of responders (e.g. seizure reduction $<50 \%$, from $50 \%$ to $75 \%$, and $>75 \%$ ) retention rate (persistence), seizure severity and Quality of Life $(\mathrm{QOL})^{2}$. The Dutch authority for registration (CBG) uses the EMEA guidelines ${ }^{3}$.

\section{International League Against Epilepsy (ILAE)}

The Commission on Outcome Measurement in Epilepsy (division of the International League Against Epilepsy) has developed guidelines based on the duration of a trial ${ }^{4}$. This commission states that in short-term studies time-to- $n$ th-seizure after randomisation or after reaching the predefined maximum dose is the outcome measure of first choice. In long-term studies time to 1-year seizure remission (or for a shorter or longer period), frequency or number of seizures over predetermined intervals and proportion of patients remaining seizure-free after achievement of the initial target maintenance dosage should be used. Further, this commission advises to monitor adverse effects more closely. Adverse effects of medication have a much larger negative impact on peoples' QOL than do (fear of having) seizures ${ }^{4}$.

\section{Food and Drug Administration (FDA)}

The Food and Drug Administration (FDA) in the USA has also developed its own guidance on the topic of drug evaluation and research ${ }^{5}$. The FDA states that there are six methods for measuring efficacy of antiepileptic drugs. These efficacy measures 
Table 1 Primary endpoints to be used in clinical trials of anti-epileptic drugs.

\begin{tabular}{lccc}
\hline & EMEA & FDA & ILAE \\
\hline Seizure frequency & + & + & + \\
Decreased total seizure time & & + & \\
Increased seizure free intervals & & + & \\
Improved functional capacity & & + \\
Decreased incidence of adverse effects & + & + \\
Decreased generalization of focal seizures & & + \\
Time to $n^{\text {th }}$ seizure & & \\
Percentage of patients remaining seizure free for at least 6 months & + & \\
Treatment retention time & + & \\
Time to 1 year seizure remission & & \\
\hline
\end{tabular}

are: 1 . reduced seizure frequency, 2 . increased seizure-free intervals/time between seizure clusters, 3. decreased total seizure time, 4. improved functional capacity, 5. decreased incidence of adverse reactions and 6. decreased generalization of focal seizures. To allow accurate and reliable recording of these outcome measures pretrial observations have to be obtained ${ }^{5}$.

Besides using these measures of efficacy, the FDA recommends serum level determinations of both the investigational and concomitant antiepileptic drugs. This has to be done to gain information about therapeutic and toxic ranges of the investigational drug and to study pharmacokinetic interactions with the concomitant antiepileptic drugs.

The primary endpoints in the guidelines from EMEA, FDA and ILAE are shown in table 1.

\section{Limitations of regulatory trials}

Patient population in the trial

Most regulatory trials are performed in patients with partial epilepsy and complex partial seizures, with or without secondary generalization ${ }^{6}$. As a result, many AEDs have been approved for treatment of partial seizures and comparatively few for other types, such as absence, myoclonus and infantile spasms.

Patients who are included in add-on trials of new AEDs have very frequent seizures, a long duration of epilepsy and have failed numerous AEDs . Inclusion criteria usually require a minimum of 3 to 4 complex partial or secondarily generalized seizures per month. Often seizure frequency is even higher in many patients included in such trials. The use of such patients may negatively bias the true effectiveness of a new AED. 
Most clinical trials study patients between 18 and 65 years of age. The first peak of incidence of epilepsy however, is in the first decade of life ${ }^{7}$. In the first year of life the incidence is approximately 120 per 100,000, decreasing to 40 per 100,000 for the remainder of the first decade. The cause of epilepsy is often prenatal or a consequence of acute circulatory failure. The second peak of incidence is in the elderly. The incidence rises to approximately 120 per 100,000 in those older than 60 years. Causes of epilepsy at that age are often stroke, brain tumor, head injury and Alzheimer's disease. This leaves us with questions whether the results of the regulatory trials can be generalized to the patient groups with highest incidence, i.e. children and elderly.

\section{Duration of the trial}

The most important limitation of regulatory trials, though, is its short duration, considering the chronic use of a AED in clinical practice. It leaves us with questions how effectiveness is maintained over time, whether tolerance occurs, how side effects resolve after adaption and whether new side effects develop after continuous use. Patients who became seizure-free cannot be followed after completion of the trial. This is a problem because the goal of the anti-epileptic drug therapy is to become seizure-free. A short-term remission of the seizure activity is not what the patient asks for and can actually introduce new problems for the patients in terms of adapting to the condition. Moreover antiepileptic treatment is usually chronic treatment, i.e. treatment over periods of many years. In such periods other types of side-effects may occur than in the limited period of a drug trial. One example is osteoporosis that may occur after extended periods of treatment with enzyme inducing AEDs. Other examples are excessive weight gain or weight loss, hormonal changes, hair loss and effects on mood and behaviour. Safety problems may also arise when side effects are serious but uncommon. Rare idiosyncratic side effects and teratogenicity will be detected only after widespread use.

Trials from the surgical withdrawal design are considered too short to provide any information on tolerability problems. Results of these trials can therefore not be extrapolated to chronic use.

\section{Trial protocol versus clinical practice}

In trials patients are often titrated to fixed doses which may be too high or too low for that individual. This design does not allow patients to reach their own best dose. The results of these trials may therefore both underestimate the efficacy of the tested AEDs or overestimate the adverse effects. Note also that the dose requirements for refractory patients in add-on trials may differ from the dose-requirements for newly diagnosed patients in monotherapy trials. This is due both to the difference in patient characteristics and to the absence of drug-drug interactions in newly diagnosed patients. 
Most anti-epileptic drugs must be carefully titrated to an effective dose. When the trial is designed the chosen titration scheme is not "rocket science" but an educated guess. Fast titration schemes reduce the cost of a clinical trial but can lead to higher drop out rates. In clinical practice titration schemes are often slower than in regulatory trials. Instead of rigid titration schemes doses are adjusted individually.

In clinical practice it is common to down-titrate and later discontinue pre-existing AEDs. These pre-existing AEDs may either induce or inhibit the metabolism of the new AED. Hence, upon withdrawal changes in serum level of the new AED can be expected. This leaves us with the question whether the dose of new AED should be changed in order to maintain the same serum level. Regulatory trials usually cannot provide this information.

Side effects that are unpleasant may limit the usefullness of a new AED. These can occur in a high percentage in add-on trials. When pre-existing AEDs are withdrawn however the incidence of these side effects can be much lower ${ }^{8}$. Most of the data on new AEDs are from regulatory add-on trials so tolerability problems may be exaggerated.

\section{Monotherapy}

\section{Designs}

Trials studying conversion to monotherapy in patients with multiple-drug treatment only provide evidence that the study drug is better than placebo. Some monotherapy trials in newly diagnosed patients use a low dose "pseudoplacebo" arm. If similar efficacy is measured in the low dose and in the high dose arm it may be concluded that the study drug is not effective. However it is also possible that both doses are effective. Other monotherapy trials use an "active control" design in which a new drug is compared with a standard drug. This design provides useful information to physicians. 


\section{Alternatives to regulatory trials}

In this section we will discuss first outcome measures for anti-epileptic drug testing. In the second part we will review new trial designs that provide useful information to physicians.

Outcome measures for anti-epileptic drug testing.

To evaluate the efficacy of AEDs, standardized outcome measures must be used. The current accepted outcome measures are 'time to $n^{\text {th }}$ seizure', 'seizure-freedom for 1 year or another time-interval, 'seizure frequency', 'proportion of responders','seizure severity', retention rate, and less frequently 'NNT or OR' (Number needed to treat and Odds ratio's) $)^{9}{ }^{10}$. Recently, more attention is given to 'quality of life' of patients and to the measurement of adverse effects. The exact definitions of the outcome measures will be given in the next sections.

An important distinction is that between effectiveness and efficacy ${ }^{10}$. To measure efficacy all outcome measures mentioned above, except retention rate, can be used. According to Mohanraj and Brodie retention rate is an excellent measure of effectiveness. Effectiveness is a function of efficacy and tolerability ${ }^{10}$. Recent developments in measuring efficacy of AEDs are focused on efficacy as well as tolerability. Measuring quality of life (QOL) and retention rate (persistence) are examples hereof. These outcome measures are better capable of giving an image of routine daily practice than do changes in seizure frequency, time to $n^{\text {th }}$ seizure and seizure severity. However, QOL and retention rate are not relevant as a proof of efficacy in regulatory trials.

The accuracy and validity of a possible outcome measure has to be considered prior to every trial. Factors that determine the usefulness of an outcome measure are design of the study, group of patients being studied, type of seizures that the patients experience ${ }^{9}$. Not every outcome measure is suitable for every study design and group of patients.

\section{Difficulties in handling seizure data}

Prior to understanding the pro's and contras of every outcome measure, one needs to know the difficulties in handling seizure data. In a vast majority of patients, seizures do not occur randomly ${ }^{11}$. Clustering of seizures is fairly common. Seizure clustering might imply that secondary alterations due to a seizure facilitate the precipitation of a next seizure similar to a positive feedback mechanism. Clusters of seizures can occur just outside the study period, or at baseline suggesting an apparent change where none has in fact occurred ${ }^{12}$. Furthermore, occurrence of seizures is very variable between and in patients ${ }^{12}$. The non-normal distribution of seizures over time makes counting and analysing seizures within a limited time-frame of a trial difficult ${ }^{12}$. The chance of counting a seizure wrongly (double or not), by physicians as well as by 
patients, is increasing when seizures occur with very small intervals. Also, changes in seizure severity are often difficult to assess ${ }^{12}$. Therefore, video recordings are more often used to achieve more reliable seizure counts. EEGs are also becoming more important e.g. when counting absence seizures ${ }^{4}$. Because of the non-normal distribution of seizures in time, parametric analytic tests are not possible. In order to use parametric tests, efficacy measures that are dichotomous or categorized have been developed.

\section{Efficacy measurement in short-term studies}

Time to $n^{\text {th }}$ seizure.

Time to $n^{\text {th }}$ seizure (=number of seizure-free days) is an efficacy measure that is valid in studies with newly diagnosed patients ${ }^{14}$. The time to a first seizure following randomisation allows immediate determination of the proportion of patients at different points in time who remain seizure-free on the agents being compared ${ }^{4}$.

The major advantage of this outcome measure is the protective effect for patients. As soon as a one, maximal two or three seizure(s) have occurred patients are withdrawn from the study ${ }^{15}$. Furthermore, this outcome measure has the largest statistical power in studies in newly diagnosed patients ${ }^{16}$. This endpoint may, however, lack clinical significance because the goal for the treatment of patients is long-term seizure freedom ${ }^{16}$.

Unfortunately, when restricting the number of seizures that a patient is allowed to experience, the power of the trial declines ${ }^{10}$. Another pitfall in using this outcome measure is that little information about optimal dosing regimen and time needed to reach a maximum effect is known ${ }^{10}$.

\section{Change in seizure frequency/Proportion of responders}

When analysed as a continuous variable, seizure frequency is the most sensitive measure of efficacy and should be used whenever possible. However, in studies with newly diagnosed patients it is a less relevant efficacy measure as most of these patients have had only a few seizures ${ }^{16}$.

To allow a reliable interpretation of this efficacy measure it is important that the period of time during which seizures are counted is known prior to the start of the trial. Measurement during a too short timeframe does not render clinically significant results ${ }^{4}$.

Percentage reduction in seizure frequency can be used when seizure counts exhibit a wide range of variation. Mostly a reduction of $>50 \%$ or $>75 \%$ is used here (this is called responder rate). This distinction is arbitrary and may miss important differences between treatments when placed at an insensitive cut-off point. Using a categorization of the seizure frequency, e.g. $0-19 \%, 20-39 \%, 40-59 \%, 60-79 \%, 80-99 \%$ 
and $100 \%$ can be a better alternative measure. It is more informative and less likely to mislead readers ${ }^{10}$.

It is an advantage that a seizure frequency reduction of more than $50 \%$ has been used in many studies, thus enabling comparison among AEDs. Note that the seizure frequency can increase (i.e. $>100 \%$ ) after the introduction of a new AED. This should be measured as an adverse effect/non-responder.

\section{Efficacy measurement in long-term studies}

\section{Seizure freedom}

The primary goal of treatment with AEDs is the achievement of seizure freedom. This is based on the assumption that most patients only have a significantly improved quality of life when full seizure remission is achieved ${ }^{13}$, 14 . This measure of efficacy is however only appropriate in long-term studies as the clinical relevance of this parameter strongly depends on the (predefined) time interval during which patients have to be free from seizures. Freedom from seizures during a few weeks (often used as a criterion in 12 weeks trials) can hardly be called an improvement, also given the non-parametric distribution of seizures in individual patients.

A major disadvantage of this outcome parameter is that a reliable determination of seizure freedom of e.g. one year has to be done using a follow up period of at least three years ${ }^{4}$.

\section{Retention rate (persistence)}

Retention rate is an excellent parameter to measure both efficacy and tolerability. Retention rate is a function of efficacy and tolerability (e.g. adverse events) ${ }^{4}, 10$. Persistence is the time that patients continue to use an AED, when in fact they need one . It is being presented as that part of all patients who have had a prescription for the drug that is still using it after a predefined period of time. Although this approach is less suitable for the current regulatory trials, it conforms to everyday practice and can provide useful clinical information. A drug that is only modestly efficacious but has excellent tolerability might fare better than one that is more efficacious but is more prone to produce side effects ${ }^{10}$. Therefore, this efficacy parameter is hardly ever used in trials that are conducted for a proof of efficacy.

\section{Seizure severity}

Even if a treatment does not control seizures completely, reduction in severity can often be achieved. The severity of seizures and their clinical manifestation is often of equal or greater impact on patient well-being than is the total number of seizures ${ }^{4}$. 
Three scales are available to measure the severity of seizures. These are the Veterans Administration (VA) Seizure Severity and Frequency Rating Scale, the Liverpool Seizure Severity Scale and the National Hospital Seizure Severity Scale(NHS3) ${ }^{4}$.

The VA scale was the first scale developed specifically for clinical trials to measure seizure severity. The rating is modified by a variety of factors reported by patients as important in determining the severity of their seizures, e.g. sleep deprivation, warning/aura, nocturnal timing, fever or illness, and missed doses of AEDs ${ }^{4}$.

The Liverpool Seizure Severity Scale was developed on the assumption that seizure severity is based on two main factors: patients' perceptions of control over their seizures (Percept subscale) and severity of ictal and postictal phenomena (Ictal subscale). Patients rate major and minor seizures on a 5-point scale. This scale needs further evidence of sensitivity before it can be accepted as a reliable method for assessing the severity of seizures in clinical trials ${ }^{4}$.

The NHS3 assigns a score to seizures based on interference with patient function. This scale uses information gathered from the patient and witnesses, if available, during a structured interview ${ }^{4}$.

There are several problems associated with these scales. Seizure severity may be evaluated differently by the physician and the patient. Second, the scales have only validity when used in a prospective study design. If researchers use these scales in a retrospective design, they largely become dependent of patients' memories. The major advantage of these scales is though that the outcomes are highly associated with patients' perceived quality of life and thus gives different results compared to other efficacy measures.

Although the psychometric properties of these scales are well established, there is little evidence to support their clinical utility.

\section{Number Needed to Treat (NNT) and Odds Ratio (OR)}

These efficacy measures are not used very often in trials with AEDs. Thus these two outcome measures can not be used to compare studies.

However, in meta-analyses the OR is an extremely efficient outcome measure. The advantage of NNT is that it is well understood by physicians. It provides an estimate on how many patients they need to treat with the AED to prohibit one event. Maybe in the future NNT can be used in relation to the efficacy measures mentioned above. By coupling the NNT to one of the regular outcome measures, both regulatory authorities and physicians are satisfied.

Quality of life

Health related quality of life measurements have been an area of increasing interest in recent years. However, quality of life is heavily dependent on seizure freedom and is thus unlikely to be an independent outcome variable in clinical trials. Furthermore, 
it will not be accepted as a proof of efficacy by regulatory authorities, because it is a highly subjective measurement.

A number of health related quality of life measures (for epilepsy) have been developed over the last several years. These measures enable assessment of patients' perception of the impact of their disease on their social, mental, and physical health. Four disease-targeted instruments exist: Liverpool HRQOL Battery, ESI-55 (Epilepsy Surgery Inventory), QOLIE-31 and QOLIE-89 which is an extension of de QOLIE-31 (QOL in Epilepsy) and the Impact of Childhood Illness Scale. Generic quality of life measures that can be used are the SF-4, RAND-4 HSI scoring procedure, SF-12 which is a subset of SF-4, Nottingham Health Profile, Sickness Impact Profile (SIP), Dartmouth COOP Function Charts and EUROQOL ${ }^{4}$. The central focus among the instruments is to ascertain what types of (epilepsy-related) problems are affecting the life of the individual patient ${ }^{17}$.

\section{Measures of efficacy that can be used in the future}

\section{Monitoring adverse events}

In many patients, QOL is affected more adversely by the side effects of treatment than by the seizures themselves. Therefore, evaluation of side effects represents an essential component in the assessment of overall clinical outcome. The evaluation of adverse events is, however, burdened with problems. One is the lack of standardization in methods of detection and quantification of side-effects. In most AED trials performed to date, recording of adverse effects has been based on patient's spontaneous reporting. Spontaneous reporting has clear advantages in highlighting effects that are clinically relevant but is associated with extreme variability in sensitivity and accuracy of detection and, overall, with significant underreporting ${ }^{18}$. An alternative to spontaneous reporting is use of standardized procedures with respect to patients' questioning and physical examination ${ }^{19}$. The use of checklists ensures that specific effects are adequately explored, but may produce bias towards detection of effects that are already known or expected. Furthermore, underreporting can be solved by giving permanent attention to adverse drug reactions. This can be done by education of the medical staff, by encouraging electronic and telephone reporting (one will report sooner if reporting is easier) and by sending reminder letter ${ }^{20}$. However, performing these encouraging measures costs a lot of time and of all the work that has to be done, encouraging of reporting adverse drug reactions is generally not considered a top priority, especially by the pharmaceutical industry. A second problem is difficulty in determining the relationship between a recorded adverse event and the treatment. Unless precise guidelines are provided, physicians will differ widely in judging the likelihood of a cause-effect relationship. Patients also may not associate 
some types of subtle problems or 'covert problems' (such as cognitive effects) and the AED therapy.

One of the most clinically relevant types of side-effects, i.e. idiosyncratic reactions are extremely rare. Regulatory trials do not involve sufficient numbers of patients to uncover these events. Post-marketing surveillance is therefore extremely important in detecting these uncommon but potentially serious problems ${ }^{21}$.

A recently developed tool to measure quality of life related to side effects of AEDs is the SEALS inventory (Side Effects And Life Satisfaction). It contains 38 questions related to the patient's feelings and behaviour over the past week. The questions are grouped into five subscales termed: worry, temper, cognition, dysphoria, and tiredness. Each question is answered on a four-point scale $(0=$ never, $1=$ occasionally, $2=$ sometimes, $3=$ many times). Answers are summed to provide a score for each factor, and factor scores are totalled to generate an overall SEALS score ${ }^{22}$. Another scale is the ABNAS neurotoxicity scale, that provides estimates for the cognitive side-effects of an $\mathrm{AED}^{23}$.

\section{Antiepileptogenic properties}

The epileptic process (epileptogenesis) consists of three phases: (1) the initial insult (brain damage, genetic malformation, stroke, infection, status epilepticus), (2) the latency period during which the actual process of epileptogenesis is taking place (neuronal death, gliosis, plasticity, neurogenesis, angiogenesis, reorganization of extracellular matrix, reorganization of receptors and channels) and (3) the onset of spontaneous and recurrent seizures ${ }^{24}$. Epileptogenesis is thus the process by which a brain becomes epileptic or starts generating spontaneous seizures ${ }^{25}$. The treatment of epilepsy focuses exclusively on preventing or suppressing seizures (anticonvulsive properties), which are the end products of the epileptic process. The challenge is whether epileptogenesis can be prevented by therapeutic intervention. Achieving this goal is becoming more realistic now there is a better understanding of the neurobiology of the epileptic process and the factors that predict the risk for progression in individual patients.

Several animal models of epileptogenesis exist, but we do not know which, if any, accurately reflects the process in humans. These models include the kindling model, status epilepticus induced by chemicals and application of alumina gel, iron compounds, or penicillin to the brain ${ }^{25}$. Sometimes the kainic acid and the pilocarpine models are used ${ }^{24}$. These models have an interval between the initial insult and the development of spontaneous seizures. In the presented models interventions have been performed during this interval to see whether they decrease or delay the onset of spontaneous seizures. The efficacy measures that are used to determine antiepileptogenic properties of an AED are a change in seizure frequency and sometimes seizure severity ${ }^{24}$. 
Although laboratory models can be very helpful in elucidating mechanisms and pointing out drugs that are likely to have an antiepileptogenic effect, one needs to perform clinical trials to confirm these effects in humans. Nowadays, several trials have been performed in humans. In these trials there are no antiepileptogenic effects observed ${ }^{26}$. At present, no studies have investigated the antiepileptogenic effects of AEDs after other epileptogenic insults such as stroke or head trauma.

Another issue relates to study design. There are no evidence-based data that could be used to decide when to start treatment and how long to continue it. Studies of antiepileptogenesis are further complicated by the fact that there are no data on the molecular mechanisms of epileptogenesis after brain insults other than status epilepticus $^{24}$.

\section{Proposal for new designs in anti-epileptic drug trials}

Long-term studies are particularly useful in providing an insight into the natural history of treated epilepsy. These data can help to identify patients who are likely to achieve remission and those who have a more progressive seizure disorder. The basic requirement for any long-term outcome study is that it follows routine clinical practice as closely as possible. Exclusion criteria should be kept to a minimum ${ }^{10}$. These studies can be observational where each patient's treatment is chosen clinically. Dosing schedules should be flexible, tailoring therapy for the individual patient. This allows the therapeutic potential of each AED to be maximised ${ }^{10}$. There is an unavoidable risk of selection bias and differences in outcomes might not always be due to differences in treatment. Adjustments for identifiable variation in patient characteristics at the analysis stage can mitigate this ${ }^{10}$.

A limitation of these long-term studies is that this type of studies is expensive and labour-intensive. Therefore, the willingness to conduct this type of studies is minimal among investigators of the pharmaceutical industry. They are also not relevant in proving efficacy during the registration phase. As a consequence of these drawbacks there are not much long-term studies available.

The randomized "use" trial is even more useful' ${ }^{1}$ In this design patients are randomized to one of the new drugs, titrated to their own ideal dose, and followed over a long period of time. An example of this approach is the SANAD study ${ }^{27}$. Primary outcomes were time to treatment failure (inadequate seizure control, intolerable sideeffects or both) and the time from randomisation to a 1-year period of remission of seizures. Analysis was by both intention to treat and per protocol. The randomized "use" trial has a potential to inform clinicians on the relative tolerabilities and the relative efficacies of new anti-epileptic drugs in a clinical practice setting. Again the pharmaceutical industry is not very interested in performing these trials. The manufacturer of the old drug can not invest in such a trial because there is no return on 
investment. The manufacturer of the new drug does not want to take the risk that the new drug turns out to be equal or inferior to the old drug.

Novel add-on time-to-nth-seizure designs (ie, studies where patients exit after having a predefined number of seizures) enable patients to exit a trial when they are doing poorly and should be promoted.

Monotherapy trials with historical controls instead of a pseudoplacebo solve some ethical problems.

Uncontrolled trials can be useful to investigate drug-drug interactions and to detect signals of potential efficacy in subpopulations.

\section{DISCUSSION AND CONCLUSIONS}

Regulatory trials are necessary to prove the efficacy and the safety of new AEDs. Mostly these trials are performed in refractory patients who suffer from partial epilepsy between 18 and 65 years of age. Fast titration schemes and fixed doses are studied in short-term studies. Doses of pre-existing AEDs do not change during the trial. Therefore generalization of the results to clinical practice is difficult.

There is some room for improvement of regulatory trials. When a patient does not respond to the new AED in a monotherapy trial other treatment is delayed for at least 4-6 months. Patients can be protected against this delay by using the efficacy parameter "time-to-the-nth-seizure".

Seizure frequency reduction is still a very important efficacy parameter. More attention must be given to the non-normal distribution of seizures.

In addition to improvements in the design of regulatory trials other designs should be promoted. There is a need for long-term studies. Important efficacy parameters for these studies are seizure freedom and retention rate. More attention should be given to the monitoring of side effects because tolerability problems often lead to discontinuation of AED therapy.

Special attention should be given to the measurement of seizure freedom in longterm studies ${ }^{28}$. Usually, intention-to-treat analysis is employed with the principal of last observation carried forward when patients discontinue therapy prior to the end of the treatment period. In this way seizure freedom results are overestimated. Gazzola et al suggested that dropout by definition cannot be counted as seizure free (pragmatic intention-to-treat analysis) ${ }^{28}$.

From the clinical perspective the randomized "use" trial is very useful. In this design patients are randomized to one of the new drugs, titrated to their own ideal dose, and followed over a long period of time. The randomized "use" trial has a potential to inform clinicians on the relative tolerabilities and the relative efficacies of new anti-epileptic drugs in a clinical practice setting. It takes time however to organize 
these trials and funding is a problem. It would help if a "reward" could be offered to a sponsor, for instance prolongation of the patent of the AED. In the mean time it should be promoted that long-term studies are started right after market approval of a new AED. These studies are particularly useful in providing an insight into the natural history of treated epilepsy. These data can help identify patients who are likely to achieve remission and those who have a more progressive seizure disorder. The basic requirement for any long-term outcome study is that it follows routine clinical practice as closely as possible. Exclusion criteria should be kept to a minimum ${ }^{10}$. These studies can be observational where each patient's treatment is deliberately chosen. Dosing schedules should be flexible, tailoring therapy for the individual patient. This allows the therapeutic potential of each AED to be maximised ${ }^{10}$. 


\section{R E FER E NCES}

1. French JA and Dichter MA. New antiepileptic drug development: medical perspective. Antiepileptic Drugs fifth edition 2002.

2. European Agency for the Evaluation of Medicinal Products. Note for guidance on clinical investigation of medicinal products in the treatment of epileptic disorders. CPMP/EWP/566/98revl. London, 16 november 2000.

3. www.cbg-meb.nl, 5 April 2007.

4. Baker GA, Camfield C, Camfield P et al., Commission on Outcome Measurement in Epilepsy, 19941997: Final Report. Epilepsia 1998; 18(2): 213-171.

5. US Department of Health and Human Services, Food and Drug Administration. Guidance for the clinical evaluation of Antiepileptic Drugs (Adults and Children). 1981

6. Perucca E, French J and Bialer M. Development of new antiepileptic drugs: challenges, incentives, and recent advances. Lancet Neurol 2007;6:793-804.

7. Hauser WA. Seizure disorders: the changes with age. Epilepsia 1992;33[Suppl 4]:S6-S14.

8. Gilliam F, Vazquez B, Sackellares JC et al. An active-control trial of lamotrigine monotherapy for partial seizures. Neurology 1998;51:1018-1025.

9. French JA. Proof of efficacy trials: endpoints. Epilepsy Research 2001;45 (1-3): 53-59.

10. Mohanraj R and Brodie MJ. Measuring the efficacy of antiepileptic drugs. Seizure 2003;12(7):413-43.

11. Taubøll E, Lundervold A and Gjerstad L. Temporal distribution of seizures in epilepsy. Epilepsy Research 1991;8:153-65

12. Pierce MW and Shu VS. Handling seizure data: special problems. Epilepsy Research Suppl. 1993;10: 175-178.

13. Perucca E. Evaluation of drug treatment outcome in epilepsy: a clinical perspective. Pharmacy World \& Science 1997;19(5):217-62.

14. Walker MC, Sander JWAS. The impact of new antiepileptic drugs on the prognosis of epilepsy: Seizure freedom should be the ultimate goal. Neurology 1996;46(4):912-914.

15. Pledger GW and Sahlroot JT. Alternative analyses for antiepileptic drug trials. Epilepsy Research Suppl 1993;10:167-74.

16. Kwan P and Brodie MJ. Refractory epilepsy: a progressive, intractable but preventable condition? Seizure 2002;11:77-84.

17. Birbeck GL, Kim S, Hays RD et al. Quality of life measures in epilepsy: How well can they detect change over time? Neurology 2000;54(9):1822-7.

18. Abernethy DR, Alderman CP, Amsden GW et al. Most adverse drug reactions, even those that are serious, are under-reported to spontaneous reporting systems. Drugs \& Therapy Perspectives 2007;17(3):17-24.

19. Deckers CL, Hekster YA, Keyser A, Lammers MW, Meinardi H, Renier WO. Adverse effects in epilepsy therapy. Wait and see or go for it? Acta Neurol Scand. 1997;95(4):248-52.

20. Clarkson A, Ingleby E, Choonara I et al. A novel scheme for the reporting of adverse drug reactions. Archives of Disease in Childhood 2001;84:337-339. 
21. www.fk.cvz.nl bezocht op february 122007.

22. Gillham R, Bryant-Comstock L and Kane K. Validation of the side effect and life satisfaction (SEALS) inventory. Seizure 2000;9:458-463.

23. Aldenkamp AP, Baker G, Pieters MSM et al. The Neurotoxicity Scale; the validity of a patient-based scale, assessing neurotoxicity. Epilepsy Research 1995; 20: 229-239.

24. Pitkänen A. Drug-mediated neuroprotection and antiepileptogenesis: animal data. Neurology 2002; 59(9 Suppl 5):S27-33.

25. Temkin NR. Antiepileptogenesis and seizure prevention trials with antiepileptic drugs: meta-analysis of controlled trials. Epilepsia 2001; 42(4): 515-24.

26. Sasa M. A new frontier in epilepsy: novel antiepileptogenic drugs. Journal of Pharmacological Sciences 2006;100(5):487-94.

27. Marson AG, Al-Kharusi AM, Alwaidh M et al. The SANAD study of effectiveness of valproate, lamotrigine, or topiramate for generalised and unclassifiable epilepsy: an unblinded randomised controlled trial. Lancet 2007;369:1016-26.

28. Gazzola DM, Balcer LJ and French JA. Seizure-free outcome in randomized add-on trials of new antiepileptic drugs. Epilepsia 2007;48(7):1303-1307. 


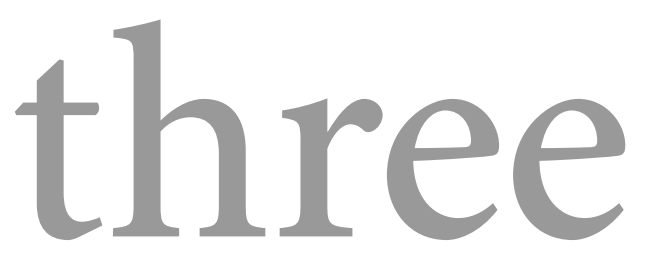

\section{Topiramate in clinical practice: long-term experience in patients with refractory epilepsy referred to a tertiary epilepsy center}




\section{A B S T R A C T}

For the treatment of patients with chronic refractory epilepsies, information about the long-term efficacy and safety profile of any new antiepileptic drug is crucial. Topiramate has been proven to be effective in patients with refractory chronic partial epilepsies in short-term controlled clinical trials, but the long-term retention, longterm efficacy, and long-term side-effect profile have not been sufficiently investigated. We analyzed all patients who had been treated with topiramate in the Epilepsy Centre Kempenhaeghe from the introduction of the drug in the spring of 1993 up to a final assessment point in mid-2002. In total, 470 patients were identified.

The data show that the clinical dose achieved was about $200 \mathrm{mg} /$ day, reached after approximately 6 months of treatment. Further dose escalation in the survivors was slow, with a mean dose of about $300 \mathrm{mg} /$ day after 24 months of treatment. Mean titration dose is $25 \mathrm{mg} /$ week, but titration strategy is mostly individual and responds to patient complaints. With respect to seizure frequency, $10-15 \%$ of the patients were seizure-free at the 6-month evaluation; 4 patients achieved a 2 -year remission. Retention rate was $53 \%$ after 1 year, $45 \%$ after 2 years, $38 \%$ after 3 years, and $30 \%$ after 4 years. At 4 years, almost $70 \%$ of the patients had discontinued to-piramate. The main reason was adverse events, which accounted for about $65 \%$ of the discontinuations. Behavioral side effects were dominant, with mental slowing (27.6\%), dysphasia (16.0\%), and mood problems (agitation: 11.9\%) being the most frequently reported side effects. In about $10 \%$ of the patients side effects led to discontinuation despite the obvious favorable effects on seizure frequency. Comparisons between the patients who discontinued topiramate treatment and those who continued topiramate showed that discontinuation was associated with comedication (vigabatrin and lamotrigine). Our conclusion is that TPM is associated with a high incidence of side effects in clinical practice, affecting long-term retention. Meaningful prognostic factors that may help us in clinical decision making, i.e., to prevent the side effects or to help us identify those at risk, have not been found. 


\section{N T RODUCT IO N}

For the $20-30 \%$ of patients with pharmacoresistant epilepsies, the recent introduction of new antiepileptic drugs (AEDs) is an important and welcome development ${ }^{1}$. One of these new AEDs is topiramate (TPM), a sulfamated monosaccharide, which has multiple mechanisms of action ${ }^{2}$. TPM has been proven to be effective in patients with refractory chronic partial epilepsies in short-term controlled clinical trials ${ }^{3}$. For patients with refractory epilepsy, however, the value of a drug in clinical practice cannot be derived simply from the outcomes of such controlled trials. For example, a mere $11 \%$ of the patients discontinued treatment in most of the controlled clinical trials, whereas this rate seems to be much higher in clinical practice ${ }^{4}$. The main reason is the relatively short duration of clinical trials - 12 weeks on average for most trials designed for registration purposes. Patients with refractory epilepsy will, however, as a rule, need long-term treatment. Therefore more information is needed about the long-term profile of a new drug in clinical practice. Several studies have shown retention rate to be the best indicator of the long-term clinical usefulness of a particular drug. Retention rate is considered to be a composite of drug efficacy and drug safety and expresses the willingness of patients to continue drug treatment. It is therefore clinically the most relevant parameter of an antiepileptic drug. The 1- year retention rate for TPM is reported to be $55 \%{ }^{6}$, comparable to the 1-year retention rates for lamotrigine (LMT, 60\%), vigabatrin (VGB, 58\%), and gabapentin (GBP, $45 \%)^{7}$. In a follow-up study, the 2 -year retention rate for TPM was $42 \%$, and the 3-year rate, $30 \%{ }^{5}$.

Previous studies have clearly shown that treatment with TPM is associated with all kinds of side effects. CNS-related side effects are the most frequently reported side effects and have the most serious impact on daily life ${ }^{4}$, especially with respect to neurocognitive effects ${ }^{8}$. Most of the side effect reports come from clinical trials and thus represent the adverse effects of the drugs in the short term and in relatively controlled circumstances. It is not clear whether the tolerability problems are longterm, although some evidence indicates that habituation for TPM occurs at a later stage than for most AEDs?.

We were particularly interested in which factors influence long-term retention. TPM was first introduced as an experimental drug in The Netherlands in 1993 and became licensed in 1999. As yet, however, the long-term profile of the drug in refractory patients is not available for clinical decision making. For our specific patient group (i.e., patients with refractory epilepsies in a tertiary epilepsy referral and care center) such data become increasingly more important as more drugs and non-pharmacological options become available. We therefore performed a systematic audit of TPM use in the "real life" setting of our center, analyzing all patients who had received or who are still using TPM. 
The study was approved by the ethics committee. All patients who had been treated with TPM in the Epilepsy Centre Kempenhaeghe from the introduction of the drug in spring 1993 up to a final assessment point mid-2002 were identified by means of our automated medical information system (MIS) and subsequently analyzed. Of the 470 patients who were identified, 37 (7.9\%) were already exposed to TPM on referral to our center. These patients were also included in the analysis, but were not used in analyzing the retention rate and the starting dose to avoid a survival bias. A standardized data form was developed. The data were obtained from our MIS and individual patient medical records. If needed, in case of any uncertainty, treating neurologists were asked for additional information. Variables that were included in the database were: patient characteristics (age, sex, weight, epilepsy duration at start of treatment with TPM, mental retardation, CT/MRI abnormalities), history of drug use, titration schedule for TPM, length of treatment with TPM, efficacy, reported side effects, and reasons for discontinuation of TPM. Epilepsy and seizures were classified using the International League Against Epilepsy (ILAE) classification. Treatment was evaluated every 6 months. If no visit was available at these assessment points, the nearest visit within 3 months was used. Efficacy was measured using a 3-point scale: increase in seizure frequency, reduction in seizure frequency, or seizure remission. Reason for discontinuation of TPM was entered into the database using a 4-point scale of no efficacy, side effects, both, and other. Data forms were entered into a computerized database for analysis. Statistical analysis was performed using SPSS 10.0 for Windows. For each point descriptives were calculated. Retention rate of TPM was calculated by using Kaplan-Meier survival analysis ${ }^{10}$. Patients who did not reach a specific assessment point because of a recent start with the drug were excluded from each time point in the retention rate analysis.

Therefore the retention rate for 1 year represents the percentage of patients who reached the 1-year point, i.e., those for whom treatment started at least 1 year earlier.

Comparisons between patients who continued on TPM and those who discontinued were analyzed using non-parametric statistics: Mann-Whitney U test for ordinal (rank) data and Pearson $\chi^{2}$.

\section{R ES U L T S}

Table 1 lists the main characteristics of the patients.

Four hundred seventy patients were identified. Thirtyseven patients (7.9\%) had already received TPM before referral to our center. The mean age of the study group 
Table 1 Patient demographics and characteristics.

\begin{tabular}{|c|c|}
\hline Total number of patients included & 470 \\
\hline Age (years) & Mean: 34.9 (sd 8.6) \\
\hline Age range & $1-73$ \\
\hline Age distribution & $115<18$ years \\
\hline $\operatorname{Sex}(F / M)$ & $225 / 245$ \\
\hline Weight (kg) & Mean: 53.8 (sd 26.9) \\
\hline \multicolumn{2}{|l|}{ Type of epilepsy } \\
\hline Localization related & $354(75.3 \%)$ \\
\hline Generalized & $94(20.0 \%)$ \\
\hline Undetermined & $10(2.1 \%)$ \\
\hline Not classified & $12(2.6 \%)$ \\
\hline \multicolumn{2}{|l|}{ Seizure type } \\
\hline Partial onset & $383(81.5 \%)$ \\
\hline Generalized (including secondarily generalized) & $219(46.6 \%)$ \\
\hline Not classified & $21(4.5 \%)$ \\
\hline Non epileptic attacks & $33(7.0 \%)$ \\
\hline Age at onset of seizures (years) & Mean: $10.2(\mathrm{sd} 11.5)$ \\
\hline Duration of epilepsy before TPM introduction (years) & Mean: 20.7 (sd 13.6) \\
\hline Mental retardation ( $\mathrm{n}$ and \%) & $166(35.3 \%)$ \\
\hline $\mathrm{CT}+/-/$ not reported ${ }^{*}$ & $86 / 109 / 275$ \\
\hline MRI +/-/not reported ${ }^{*}$ & $174 / 107 / 189$ \\
\hline TPM treatment time (days) & Mean: 415.1 (sd 448.5) \\
\hline
\end{tabular}

* $+=$ abnormalities - no abnormalities found.

was 34.9 (SD 8.6); patients were equally distributed with respect to gender. Patients had epilepsy for a mean of 20.7 years (SD 13.6). Localization related epilepsy was the most frequent type of epilepsy, while partial-onset seizures were the most frequent seizure type. CT and MRI abnormalities were seen in respectively $86(18.3 \%)$ and 174 patients (37.0\%). One hundred sixty-six patients (35.3\%) were known to be mentally retarded. Thus, the group of patients on TPM represent the typical group of refractory patients referred to a tertiary epilepsy center. In this study TPM was added to the antiepileptic drugs the patient already used. The mean length of TPM treatment for all patients (470 patients) was 415.1 (SD 448.5) days.

The mean titration dose is $23.5 \mathrm{mg} /$ week; this was calculated for the 470 patients minus the 69 patients who were in the first steps of titration at the final evaluation time point (first or second dose step) (Table 2). We also analyzed the titration schedule as this is a subject of frequent discussion among clinicians. The most frequently applied strategy is not a fixed schedule, but varies in response to patient complaints 
Table 2 Topiramate titration schedules.

\begin{tabular}{ll}
\hline Titration schedule & Frequency $^{\mathrm{a}}$ \\
\hline Individual schedules & $138(29.4 \%)$ \\
$25 \mathrm{mg} /$ week & $134(28.5 \%)$ \\
$25 \mathrm{mg} /$ week up to $100 \mathrm{mg}$ followed by $50 \mathrm{mg} /$ week & $47(10.0 \%)$ \\
$12.5 \mathrm{mg} /$ week up to $50 \mathrm{mg}$ followed by $25 \mathrm{mg} /$ week & $46(9.8 \%)$ \\
$25 \mathrm{mg} / 2$ weeks & $36(7.7 \%)$ \\
Mean titration dose/week & $23.5 \mathrm{mg} /$ week \\
\hline
\end{tabular}

${ }^{a}$ No. of patients (\%).

Table 3 Seizure reduction.

\begin{tabular}{lcccc}
\hline & $\begin{array}{l}\text { Change from } \\
\text { baseline } \\
\text { to 6 months }\end{array}$ & $\begin{array}{l}\text { Change from } \\
\text { 6 months } \\
\text { to 12 months }\end{array}$ & $\begin{array}{l}\text { Change from } \\
12 \text { months } \\
\text { to 18 months }\end{array}$ & $\begin{array}{l}\text { Change from } \\
18 \text { months } \\
\text { to 24 months }\end{array}$ \\
\hline Seizure-free (during 6 months) & $26(9.0 \%)$ & $17(9.6 \%)$ & $20(16.1 \%)$ & $13(14.6 \%)$ \\
Lower seizure frequency & $134(46.2 \%)$ & $34(19.2 \%)$ & $15(12.1 \%)$ & $11(12.4 \%)$ \\
No efficacy & $36(12.4 \%)$ & $56(31.6 \%)$ & $44(35.5 \%)$ & $20(22.5 \%)$ \\
Higher seizure frequency & $34(11.7 \%)$ & $25(14.1 \%)$ & $20(16.1 \%)$ & $17(19.1 \%)$ \\
Not reported & $60(20.7 \%)$ & $45(25.4 \%)$ & $25(20.2 \%)$ & $28(31.5 \%)$ \\
\hline
\end{tabular}

and seizure frequency (29.4\%). The most frequent fixed titration schedule is $25 \mathrm{mg} /$ week (28.5\%). A mean dose of $217.3 \mathrm{mg} /$ day is achieved at 6 months. After that, dose escalation levels off. After 24 months the mean dose is $323.1 \mathrm{mg} /$ day. The highest dose achieved in an individual was $1100 \mathrm{mg}$ /day. The longest period on TPM was 96 months (1 patient).

Seizure reduction at 24 months is summarized in Table 3. After 24 months the number of patients was too small to provide meaningful data. This shows that between 9.6 and $14.6 \%$ of the patients became seizure free (during the 6 months between each assessment point). In a larger group (about 50\%) the drug had no effect on seizure frequency or was associated with an increase in seizure frequency. Only 4 patients were seizure-free for 24 months.

The most frequently reported side effects are listed in Table 4 (with both the patient and the neurologist as sources). The data were analyzed up to 24 months of follow-up. After that, the number of patients taking the drug was too small to provide meaningful data. Table 4 lists only those complaints affecting more than $5 \%$ of the patients. Mental slowing and language problems (dysphasia) were present in a large percentage of patients at 6,12 , and 18 months, but did not occur in more than $5 \%$ of the patients at 24 months. Weight loss was reported in more than $5 \%$ of the patients only in the early stage of treatment (i.e., at 6 months). Mood problems reached the $5 \%$ threshold only at the 18-month time point (agitation), and urogenital problems, at 24 months. 
Table 4 Most frequently reported side effects.

\begin{tabular}{|c|c|c|c|}
\hline & Reported side effects & & \\
\hline 6 months & Mental slowing (13.8\%) & Dysphasia $^{1}(6.6 \%)$ & Weight loss (5.2\%) \\
\hline 12 months & Mental slowing (13.6\%) & Dysphasia $^{1}(5.7 \%)$ & \\
\hline 18 months & Mental slowing (8.1\%) & Dysphasia $^{1}(6.5 \%)$ & Mood disorders $^{2}(5.7 \%)$ \\
\hline 24 months & Urogenital complaints $^{3}(5.6 \%)$ & & \\
\hline
\end{tabular}

Table 5 Reasons for discontinuing TPM and mean duration of TPM use.

\begin{tabular}{lc}
\hline Reasons for stopping TPM & Frequency \\
\hline Lack of efficacy & $81(17.2 \%)$ \\
Adverse events & $109(23.2 \%)$ \\
Both & $66(14.0 \%)$ \\
Other & $13(2.8 \%)$ \\
Number of patients withdrawn from TPM & $269(57.2 \%)$ \\
Number of patients continuing on TPM & $201(42.8 \%)$ \\
Patients continuing on TPM/withdrawals & 584.3 days (sd: 530.8) / 287.5 days (sd: 318.9$)$ \\
(mean duration of drug treatment in days) & \\
\hline
\end{tabular}

Reaching the threshold of $5 \%$ is more subject to chance as the number of patients decreased. We therefore do not provide data after 24 months of follow-up. Additionally, we analyzed the percentages of patients with reported side effects per assessment point. At 6 months this percentage was $49.0 \%$; at 12 months, 47.2\%; at 18 months, 48.4\%; and at 24 months, $38.2 \%$.

Table 5 and Figure 1 indicate that 269 of the 470 patients discontinued TPM treatment at some point. The main reasons for drug discontinuation were adverse events in 109 of the 470 patients (23.2\%), lack of efficacy in 81 patients (17.2\%), and a combination of reasons (no efficacy and adverse events) in 66 patients (14.0\%).

Other reasons like death and pregnancy were mentioned by 13 patients $(2.8 \%)$. The mean duration of drug treatment for patients continuing TPM was 584.3 days, and for patients who discontinued TPM, 287.5 days.

The most frequently reported side effects at discontinuation (Table 6) were cognitive slowing in $27.6 \%$, dysphasia (word-finding difficulties) in $16.0 \%$, mood disorders (agitation, hyperirritability, aggression) in $11.9 \%$, and gastrointestinal complaints (nausea, vomiting, stomachache) in $10.1 \%$ of the patients. Other side effects occurred in less than $10 \%$ of the patients. Side effects that are frequently reported in other studies, such as paresthesia and weight loss, occurred in 7.5 and $6.3 \%$ of the patients who stopped TPM treatment in our study. Excessive weight loss (>10\%) was reported in 9 patients (3.4\%), nephrolithiasis was reported in 3 patients (1.1\%), and 3 patients 


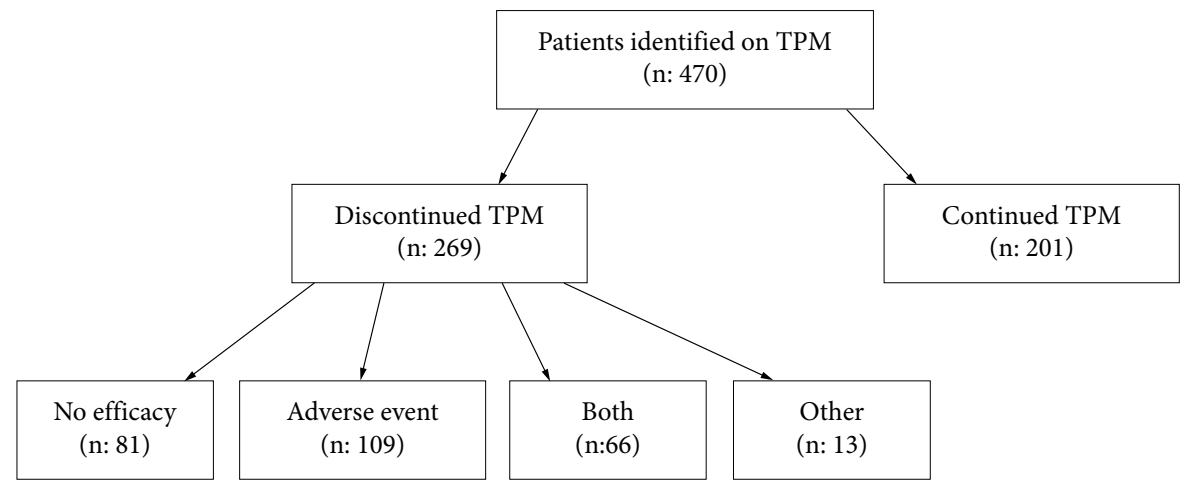

Figure 1 Flowchart of numbers of patients who discontinued and number of patients who continued TPM treatment during the evaluation period.

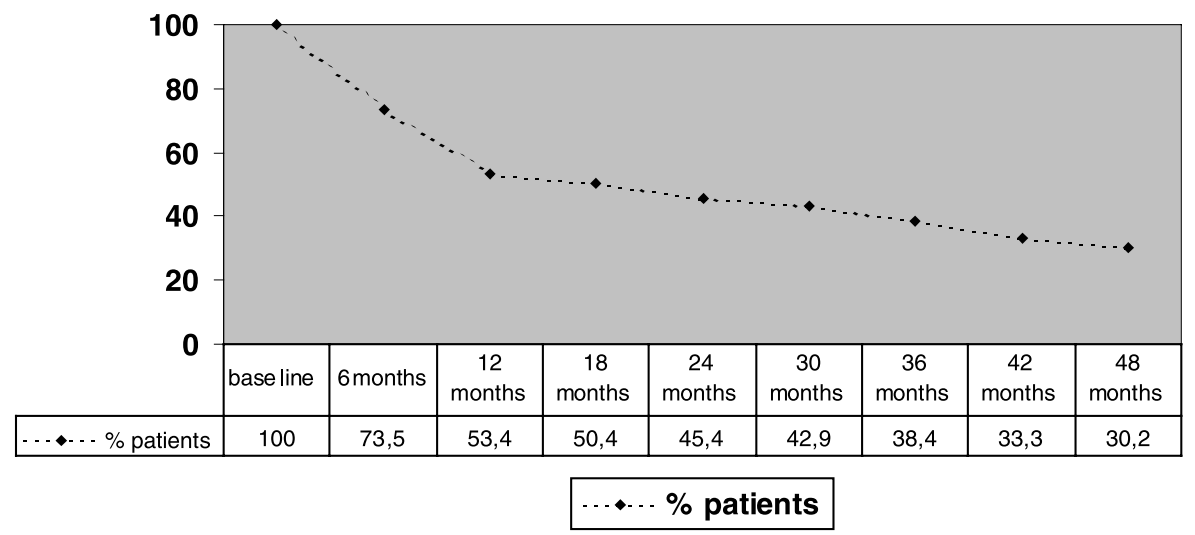

Figure 2 Kaplan-Meier cumulative survival plot for discontinuation of TPM.

(1.1\%) manifested psychosis. Cognitive dysfunction is therefore the most frequent reason for drug discontinuation.

Seven patients (2.6\%) were seizure-free when they discontinued TPM, 26 patients (9.7\%) had a lower seizure frequency, 91 patients (33.8\%) showed no efficacy, and 73 patients $(27.1 \%)$ had a higher seizure frequency.

Seventy-one patients (26.4\%) had no reported seizure reduction when stopping TPM treatment (Table 7).

Retention rate is illustrated with the Kaplan-Meier survival curve in Figure 2. The retention rate was $53.4 \%$ after 1 year, $45.4 \%$ after 2 years, $38.4 \%$ after 3 years and $30.2 \%$ after 4 years. The curve shows that the largest percentage of discontinuations 
Table 6 Reported adverse events in patients who discontinued TPM.

\begin{tabular}{|c|c|}
\hline & Frequency \\
\hline Mental slowing & $74(27.6 \%)$ \\
\hline Dysphasia (word-finding difficulties) & $43(16.0 \%)$ \\
\hline Mood disorders ${ }^{1}$ & $32(11.9 \%)$ \\
\hline Gastrointestinal complaints & $27(10.1 \%)$ \\
\hline Paresthesia & $20(7.5 \%)$ \\
\hline Skin complaints & $19(7.0 \%)$ \\
\hline Weight loss & $17(6.3 \%)$ \\
\hline Appetite loss & $17(6.3 \%)$ \\
\hline Mood disorders ${ }^{2}$ & $16(6.0 \%)$ \\
\hline Headache & $15(5.6 \%)$ \\
\hline CNS other complaints & $14(5.2 \%)$ \\
\hline Dizziness & $13(4.9 \%)$ \\
\hline Memory complaints & $12(4.5 \%)$ \\
\hline Weight loss (>10\%) & $9(3.4 \%)$ \\
\hline Non-CNS other complaints & $8(3.0 \%)$ \\
\hline Ataxia & $6(2.2 \%)$ \\
\hline Sleeping complaints & $6(2.2 \%)$ \\
\hline Muscle \& bone complaints & $6(2.2 \%)$ \\
\hline Urogenital complaints & $5(1.9 \%)$ \\
\hline Double vision & $5(1.9 \%)$ \\
\hline Eye complaints & $4(1.5 \%)$ \\
\hline Nephrolithiasis & $3(1.1 \%)$ \\
\hline Psychosis & $3(1.1 \%)$ \\
\hline Cardiovascular complaints & $3(1.1 \%)$ \\
\hline Endocrine complaints & $3(1.1 \%)$ \\
\hline Respiratory complaints & $2(0.8 \%)$ \\
\hline Weight gain & $1(0.4 \%)$ \\
\hline
\end{tabular}

${ }^{1}$ Mood disorders; agitation, aggression, hyperirritability; ${ }^{2}$ Mood disorders; depression, apathy.

Table 7 Seizure reduction in patients who discontinued TPM.

\begin{tabular}{lc}
\hline & TPM termination ${ }^{1}$ \\
\hline Seizure free & $7(2.6 \%)$ \\
Lower seizure frequency & $26(9.7 \%)$ \\
No efficacy & $91(33.8 \%)$ \\
Higher seizure frequency & $73(27.1 \%)$ \\
Not reported & $71(26.4 \%)$ \\
${ }^{1}$ Seizure reduction in the period following the last standard assessment point up to discontinuation of \\
TPM treatment.
\end{tabular}




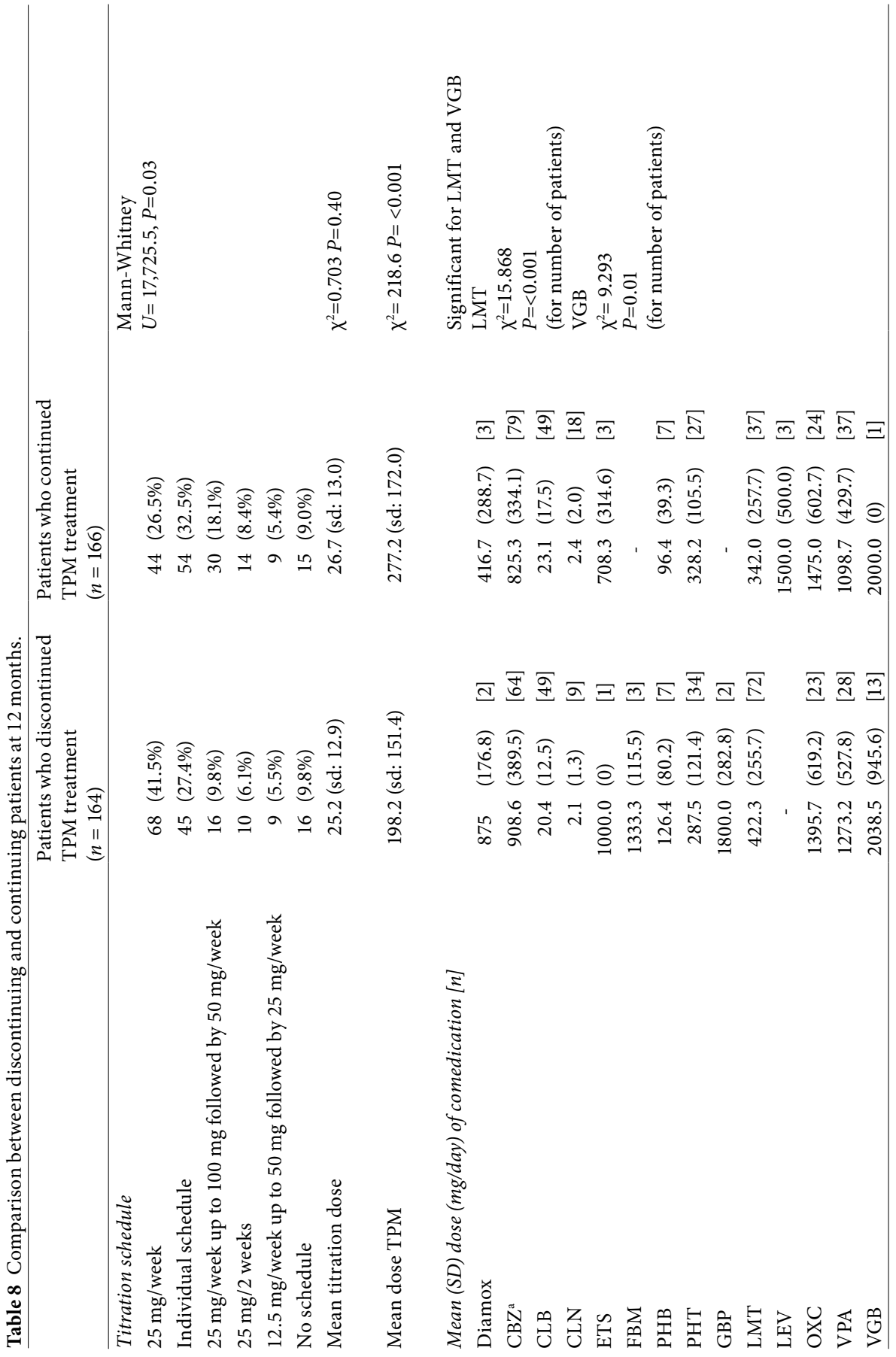



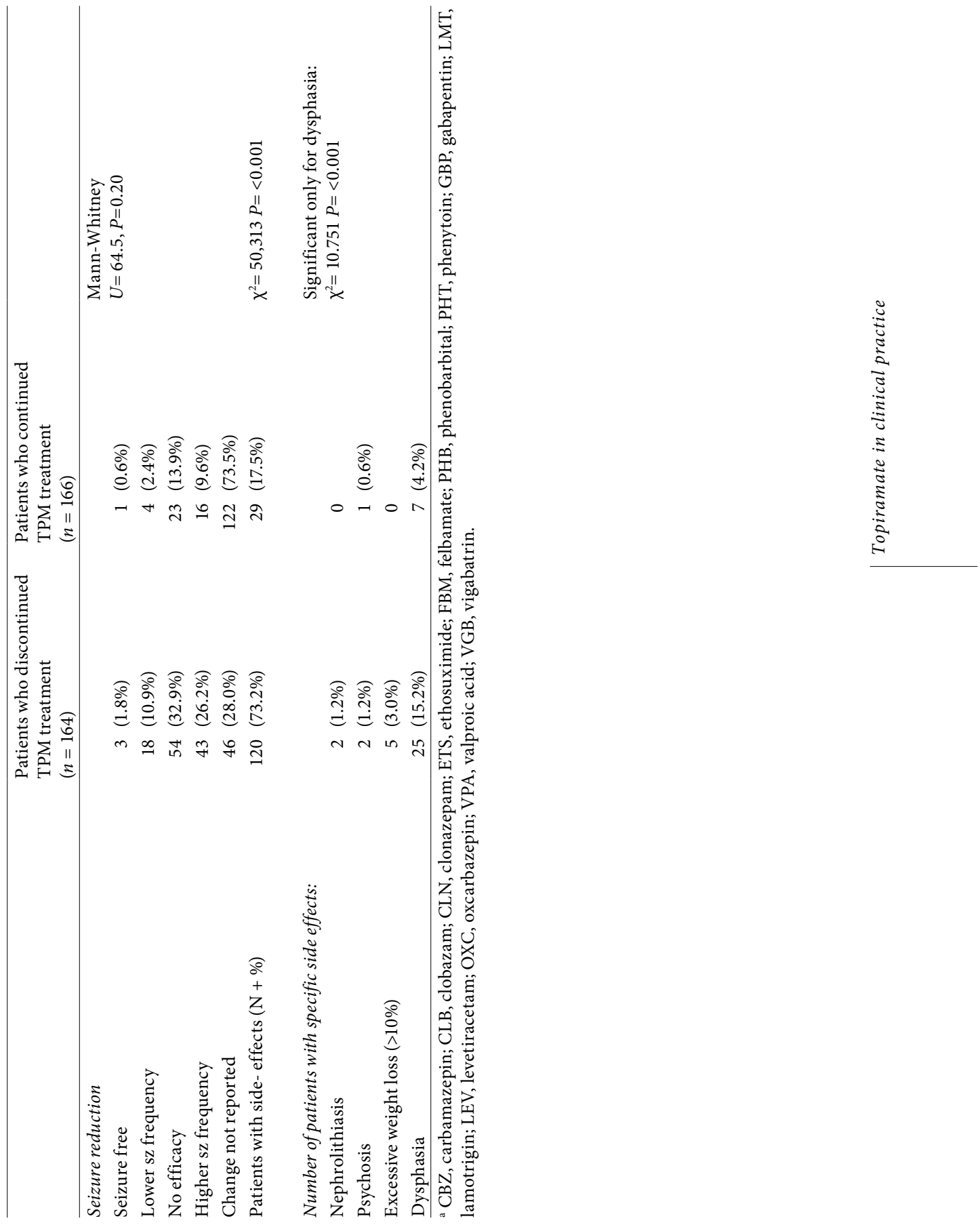
occurred in the first period of treatment (i.e., up to 12 months). After 1 year of treatment the number of discontinuations is more or less steady.

From the beginning of 1993 (first patient on TPM in our center) to the final assessment point, 269 patients discontinued TPM treatment and 201 patients continued treatment, irrespective of duration of treatment. We subsequently analyzed whether differences existed between those who discontinued the drug and those who continued TPM treatment. However, as this comparison is independent of duration of drug treatment, our analysis considered the first 12-month period and only those patients who were able to reach this assessment point $(n=330)$ (see Table 8$)$. The differences between patients who continued and those who discontinued TPM were tested for statistical significance, using $\chi^{2}$ comparisons. This analysis shows small differences in titration strategy that do not reach statistical significance; also, the mean titration doses of the two groups were similar. The mean dose of TPM in patients continuing TPM is significantly higher than in patients who discontinued TPM treatment.

When comedication was investigated, lamotrigine and vigabatrin were more frequently used as comedication in the discontinuation group. There is no significant difference in seizure control between the patient groups, although there is a trend that the patients who discontinued TPM had better seizure control. The major difference concerns the occurrence of side effects, for which there was a highly significant difference between the two groups, with more than four times the number of patients who discontinued TPM reporting side effects. Of the four most frequently discussed side effects in clinical practice excessive weight loss, nephrolithiasis, dysphasia, and psychosis, only dysphasia occurred statistically significantly more often in our patients who discontinued TPM.

\section{I S C U S I O N}

The clinical experience with topiramate has been reported in a few studies ${ }^{6,8}$. The added value of this study is the large number of patients included $(n=470)$, the long period of evaluation (almost 10 years, from first patient in 1993 up to our assessment in 2002), and the unselected group (i.e., all patients who received TPM treatment in our center). Our study produced results that may be used in clinical practice:

First, the clinical dose achieved in our patient group was about $200 \mathrm{mg} /$ day and was reached after approximately 6 months of treatment. Further dose escalation in the survivors was slow to a maximum of about $300 \mathrm{mg} /$ day after 24 months of treatment. This relatively low dose was also reported in the U.K. clinical experience report $^{5}$, which stated an average dose of about $300 \mathrm{mg} /$ day after 1 year of treatment. This is much lower than the original doses used in the clinical trials and the official dosing information and also lower than the U.S. experience reported by the PADS 
group $^{8}$ with an average dose almost twice as high $(385 \mathrm{mg} /$ day) after 6 months. This possibly reflects the opinion of many clinicians in Europe that some of the side effects are dose-related and lower dosages are preferable. The mean titration dose $(25 \mathrm{mg} /$ week) follows the current recommendations, but most neurologists do not follow the recommended fixed strategy of $25 \mathrm{mg} /$ week dose escalation up to clinical dose, but rather individualize the approach in response to patient complaints and seizure frequency. Employing such a strategy means that sometimes the dose remains the same for 4 weeks or even several months before patient and neurologist decide to escalate further. Higher starting dose and large titration steps were almost not reported in our study group. Only some of the patients who started TPM before 1999 received a higher starting dose and underwent larger dose escalation steps. Also, the titration speed is lower $\left(23.5 \mathrm{mg} /\right.$ week) than the speed used in the U.S. PADS group ${ }^{8}$ (with an average titration dose of $35.5 \mathrm{mg} /$ week).

Second, the effects of TPM on seizure frequency are rather impressive in our refractory group. If we use 6-month evaluations, about $10-15 \%$ of the patients become seizure-free. In about $30 \%$ of the patients the drug has a reported favorable effect on seizure frequency.

Third, in line with reports of comparable clinical evaluation studies ${ }^{8}$, cognitive impairments are the most frequently reported side effects of TPM treatment, specifically cognitive slowing and dysphasia (word-finding difficulties). The relevance of the reported side effects is illustrated when we inspect long-term retention rates. In total, 269 of the 470 patients stopped TPM at some point. Our study recorded a $53.4 \%$ retention rate after 1 year, $45.4 \%$ after 2 years, $38.4 \%$ after 3 years, and $30.2 \%$ after 4 years. These data are similar to earlier reported retention rates of 55, 42, and $30 \%$ from 1-, 2-, and 3-year follow-up studies ${ }^{5-7}$. By 4 years almost $70 \%$ of the patients had discontinued TPM. The Kaplan-Meier survival curve shows that the largest percentage of withdrawals occurred in the first period of treatment (i.e., up to 12 months). After 1 year of treatment the number of withdrawals more or less remained steady at a lower rate. This suggests that surviving the early stage of drug introduction (6 months) is a good indicator of long-term retention.

Fourth, the main reasons for drug discontinuation were adverse events: In 109 of the 470 patients $(23.2 \%)$, side effects were the only reason for discontinuation, and in 66 patients (14.0\%) discontinuation was decided because of a combination of lack of efficacy and adverse events. Therefore, side effects played a role in discontinuation of TPM in almost $40 \%$ of the total study group. When only those patients who discontinued TPM are considered, side effects are the only or one of the reasons for $65 \%$ of the discontinuations. When the types of side effects that led to discontinuation are considered, then undoubtedly behavioral side effects are dominant, with mental slowing (27.6\%), dysphasia (16.0\%), and mood problems (agitation: $11.9 \%$ ) being the most frequently reported. In about $10 \%$ of patients, side effects led to discontinua- 
tion despite the obvious favorable effects on seizure frequency. In these patients the side effects can therefore be considered very serious. It must be emphasized that the severity of behavioral side effects is uncommon in AED treatment ${ }^{11}$. Side effects frequently discussed in clinical practice were less frequent in this study: paresthesia was reported by $7.5 \%$ of those who discontinued TPM (this is confirmed in other reports of patients using combination therapy); large percentages are reported only in monotherapy ${ }^{4}$. Excessive weight loss was reported for $3.4 \%$ of the patients, nephrolithiasis for $1.1 \%$, and psychosis for $1.1 \%$ of the patients.

Fifth, an important aspect of our evaluation was analysis of differences between patients who discontinued TPM therapy and patients who continued treatment. Such a comparison could provide factors prognostic of TPM failure that could be used in clinical decision making. To avoid artifacts due to survival bias or small samples, this analysis was performed for the 12-month assessment point and only for those patients who were able to reach that point, i.e., for whom treatment started at least 1 year earlier. The 1-year point provides sufficient information on long-term effects as this represents the period in which a clinical dose is established and habituation occurs. This yields two groups: the discontinuation group contained 164 patients, and the continuation group, 166 patients. Some important conclusions may be drawn from our comparisons. Although the U.S. PADS study ${ }^{8}$ showed a relationship between titration speed and discontinuation our study did not show such a relationship. This is probably due to the higher titration speed used in the U.S. PADS group compared with our study group. Nonetheless our lower titration speed did not prevent discontinuation due to cognitive impairment. Therefore, our earlier suggestion that slow titration (25 mg per week) may prevent most of the cognitive side effects ${ }^{9,12}$ does not hold in clinical practice. Similar to the U.S. PADS study ${ }^{8}$, the dose in patients who continued TPM was statistically significantly higher than that in patients who stopped TPM, but this is due to an artifact, caused by survival bias: further dose escalation was possible only in those patients who continued medication. The more frequent use of lamotrigine and vigabatrin as comedication in the patients who stopped TPM is interesting but is difficult to interpret in an observational study. The patients who discontinued TPM did not, however, seem to have a more refractory epilepsy than the patients who continued on the drug. This has also been reported by the U.S. PADS study ${ }^{8}$. The only major difference is the occurrence of side effects, with cognitive side effects being predominant. This finding is not very helpful for clinical decision making, i.e., in preventing those side effects.

Similar to other observational studies the major conclusion of this study is therefore that TPM treatment is frequently associated with side effects, with cognitive impairment as the dominant patient complaint. These side effects are clinically relevant because they often require drug discontinuation, even when the drug has a favorable effect on seizure frequency. We did not find meaningful prognostic factors 
that may help us in clinical decision making, i.e. help us to prevent those side effects or help us to identify those at risk. Our earlier suggestion ${ }^{9,12}$ that slower titration, a low starting dose (25 mg), and slow dose escalation ( $25 \mathrm{mg} /$ dose-escalation steps), as well as a lower maintenance dose $(200 \mathrm{mg} /$ day), may prevent most of the side effects was not confirmed in this study. On the contrary, we found a similar pattern of longterm retention, efficacy, and side effect profile as reported in the U.S. PADS study, which used a higher titration speed and higher maintenance dose.

\section{Acknowledgment}

We thank Gloria Roberts for revising the English text. 


\section{R E FER E NCES}

1. Stefan H, Krämer G, Mamoli B, editors. Challenge epilepsy: new antiepileptic drugs. Berlin: Blackwell Science; 1998.

2. White HS. Clinical significance of animal seizure models and mechanism of action studies of potential antiepileptic drugs. Epilepsia 1997;38(Suppl. 1):S9-17.

3. Faught E, Wilder BJ, Ramsay RE, et al. Topiramate placebo-controlled dose-ranging trial in refractory partial epilepsy using 200-, 400-, 600 mg dosages. Neurology 1996;46:1684-90.

4. Rosenfeld WE. Topiramate: a review of preclinical pharmacokinetic and clinical data. Clin Ther 1997;19:1294-308

5. Lhatoo SD, Wong ICK, Sander JWAS. Prognostic factors affecting long-term retention of topiramate in patients with chronic epilepsy. Epilepsia 2000;41:338-41.

6. Kellet MW, Smith DF, Stockton PA, Chadwick DW. Topiramate in clinical practice: first year's postlicensing experience in a specialist epilepsy clinic. J Neurol Neurosurg Psychiatr 1999;66:759-63.

7. Wong ICK. New antiepileptic drugs: study suggests that a quarter of patients will still be taken the new drugs after six years. Br Med J 1997;314:603-4.

8. Tatum WO, French JA, Faught E, et al. Postmarketing experience with topiramate and cognition. Epilepsia 2001;42: 1134-40.

9. Aldenkamp AP. Cognitive effects of topiramate, gabapentin and lamotrigine in healthy young adults. Neurology 2000;54: 270-2.

10. Kaplan EL. Non-parametric estimation from incomplete obser vations. Am Stat Assoc J 1958;53:45781.

11. Vermeulen J, Aldenkamp AP. Cognitive side-effects of chronic antiepileptic drug treatment: a review of 25 years of research. Epilepsy Res 1995;22:65-95.

12. Aldenkamp AP, Baker G, Mulder OG, et al. A multicentre randomized clinical study to evaluate the effect on cognitive function of topiramate compared with valproate as add-on therapy to carbamazepine in patients with partial-onset seizures. Epilepsia 2000;41:1167-78. 


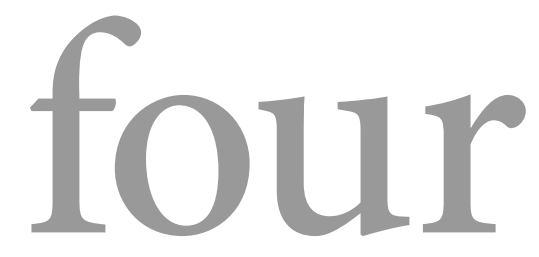

\section{Levetiracetam in clinical practice: long-term experience in patients with refractory epilepsy referred to a tertiary epilepsy center}




\section{A B S T R A C T}

Objective. For the treatment of patients with chronic refractory epilepsies, development of new antiepileptic drugs is crucial. Three regulatory trials have demonstrated that add-on levetiracetam is efficacious in patients with localization-related epilepsy. However, results from these highly controlled short-term clinical trials cannot simply be extrapolated to everyday clinical practice. Therefore, more information is needed about the long-term profile of a new antiepileptic drug in clinical practice.

Method. We analyzed all patients who had been treated with levetiracetam in the Epilepsy Centre Kempenhaeghe from the introduction of the drug in early 2001 up to a final assessment point, at the end of 2003 , using a medical information system.

Results. In total, 301 patients were included. One hundred thirty-eight patients (45.8\%) discontinued LEV treatment during the 24- month follow-up period. Reasons for discontinuation were lack of efficacy in $15.9 \%$ of patients, adverse events in $6.0 \%$ of patients, and a combination of lack of efficacy and adverse events in $16.3 \%$ of patients. By Kaplan-Meier survival analysis, the continuation rate was $65.6 \%$ after 1 year and $45.8 \%$ after 2 years. About $15 \%$ of patients in this highly refractory group had a 3-month remission, whereas $10 \%$ of patients became seizure-free for longer periods. The most frequently reported side effects at the time of discontinuation were mood disorders, tiredness, and sleepiness. Variables predicting (dis)continuation of levetiracetam treatment could not be identified.

Conclusion. Levetiracetam is a new antiepileptic drug that appears to be a useful add-on treatment in patients with refractory epilepsy. Its side effect profile is mild, with mood disorders being the most dominant adverse event. 


\section{N T ROD UCT IO N}

Epilepsy, one of the most prevalent neurological disorders, affects about $1 \%$ of the world's population. It is a chronic condition that is often difficult to treat, because $20-30 \%$ of patients have a refractory form. Therefore, the introduction of new antiepileptic drugs (AEDs) in the nineties could be an important development for this group of patients ${ }^{1}$.

One of those recently introduced AEDs is levetiracetam (LEV), an S-enantiomer pyrrolidine derivative that acts by binding to and modulating the synaptic vesicle protein $\mathrm{SV}_{2} \mathrm{~A}^{2}$ and differs, therefore, from the other AEDs, with possible consequences on efficacy and safety profile. However, its exact mechanism of action remains unknown.

LEV is rapidly and almost completely absorbed following oral administration; it exhibits linear pharmacokinetics, it is excreted largely unchanged by the kidneys, and because it has less than $10 \%$ plasma protein binding and is not cytochrome $\mathrm{P} 450$ dependent, LEV is unlikely to produce drug interactions ${ }^{3}$.

Three pivotal trials have demonstrated that add-on LEV is efficacious in patients with localization-related epilepsy ${ }^{4-6}$. However, these trials were focused on regulatory purposes and indicate only that the drug is more efficacious than placebo and that the safety profile is favorable in the short term. The most frequently reported side effects of LEV in clinical trials were asthenia, headache, somnolence, dizziness, and infection. These data reflect the side effect profile of the drug in the short term and in controlled circumstances.

We must critically evaluate the effects in clinical practice, which requires longterm use and combination therapy in many patients with refractory epilepsy. Several studies have mentioned the problems in extrapolating results from regulatory trials to everyday clinical practice?.

Among those problems are the strictly defined inclusion and exclusion criteria in drug trials. Some patients with refractory epilepsy are always excluded from these regulatory trials, especially patients with a mental handicap, who constitute an important group of patients with refractory epilepsy in a tertiary epilepsy clinic. The safety and efficacy of new AEDs in this group of patients are thus largely unknown. Moreover, drug titration schedules in clinical trials are very rigid, leading to specific problems.

For example, some of the adverse events reported in topiramate trials may have been due partly to the rapid titration that occurred during these trials ${ }^{8}$. At present, slower titration and individual titration schedules are recommended for clinical use of topiramate.

Therefore, more information is needed about the long-term profile of a new AED in clinical practice. The anecdote that safety problems developed after launch of 
three new AEDs (progabide, felbamate, vigabatrin) despite years of clinical trials demonstrates that other study designs with a long follow-up of several years can be of additional value in the research on new AEDs.

Finally, seizure freedom should be the ultimate goal in the treatment of refractory epilepsy ${ }^{9}$. In clinical trials, the primary outcome measure for new AEDs as add-on therapy has become a greater than $50 \%$ reduction in seizure frequency. This surrogate measure of efficacy indicates only that the drug has an anticonvulsive effect; this does not mean that this outcome measure is clinically important. Patients whose seizures are reduced from four to one per month are still not free from the stigma of epilepsy and its psychosocial consequences.

To date, the long-term profile of LEV in terms of efficacy and tolerability is not available for clinical decision making, although several studies mention data that suggest a similar favorable efficacy and tolerability profile as presented in clinical trials ${ }^{10,11}$. For our specific patient group, such data become increasingly more important as more treatment options become available. We therefore performed a systematic audit of LEV use in the "real-life" setting of a tertiary epilepsy center. The aim of this study was to determine the long-term effects of LEV, specifically retention time, tolerability problems, and efficacy in clinical practice.

\section{METHOD}

The study was approved by the Medical Ethics Committee. All patients who had been treated with LEV in the Epilepsy Centre Kempenhaeghe from the introduction of the drug in early 2001 to a final assessment point at the end of 2003 were identified by means of our automated medical information system (MIS) and subsequently analyzed.

A standardized data form was developed. The data were obtained from our MIS and individual patient medical records. Variables included in the database were: patient characteristics (age, sex, weight, age at onset of seizures, duration of epilepsy at the introduction of treatment with LEV, mental retardation [IQ $<70$ ], CT/MRI abnormalities), history of drug use, titration schedule for LEV, length of treatment with LEV, efficacy, reported side effects, and reasons for discontinuation of LEV. Epilepsy and seizures were classified using the International League Against Epilepsy (ILAE) classification. The percentages for seizure type refer to the proportions of patients who had each particular seizure type. Treatment was evaluated every 3 months up to 24 months.

At 21 months an insufficient number of patients remained to provide meaningful data. If no visit was available at these assessment points, the nearest visit within 1.5 months was used. Efficacy was measured using a 5-point scale: seizure remission, 
any reduction in seizure frequency, no efficacy, any increase in seizure frequency, or not classified. Reason for discontinuation of LEV was entered into the database using a 4-point scale: no efficacy, adverse events, both, or other. Adverse events were derived from the neurologist's notes in medical files. Mood disorders due to use of AEDs were classified into activating and deactivating effects, with the former leading to aggression, hyperirritability, and agitation, and the latter leading to apathy and depression ${ }^{12,13}$.

Data forms were entered into a computerized database for analysis. Statistical analysis was performed using SPSS 14.0 for Windows. The retention rate of LEV was calculated using Kaplan-Meier survival analysis ${ }^{14}$. Comparisons between patients who continued on LEV and those who discontinued were analyzed using an independent $t$ test and Pearson's $\chi^{2}$.

R E S U L T S

\section{Demographics}

Of the 304 patients who were identified, 3 were excluded: one patient had already been exposed to LEV on referral to our center, and for two patients, data were lacking.

Table 1 lists the main characteristics of the patients. The mean age was 32.5 (SD 16.8 ) years, with almost $20 \%$ being children ( $<18$ years old). There was an equal distribution with respect to gender. The mean age at onset of seizures was 11.7 (SD 11.7), with a mean duration of epilepsy of 21.3 (SD 12.9) years before introduction of LEV. Almost one in three patients (32.6\%) was mentally retarded. CT abnormalities were recorded in $17.3 \%$ of patients, and MRI abnormalities in $31.9 \%$. Localization-related epilepsy was the most prevalent epilepsy in the study group (72.1\%), with partial onset seizures being the most frequent type of seizure (80.7\%). Five patients $(1.7 \%)$ received LEV as monotherapy, 195 (64.8\%) were taking one or two other concomitant AEDs, and 101 (33.6\%) were taking three or more other concomitant AEDs. Thus, the group of patients on LEV represent the typical group of patients with refractory epilepsy referred to a tertiary epilepsy center.

\section{Long-term retention}

One hundred thirty-eight patients (45.8\%) discontinued LEV treatment sometime during the 24-month follow-up period (Table 2). Reasons for discontinuation were lack of efficacy in $15.9 \%$ of patients, adverse events in $6.0 \%$ of patients, and a combination of lack of efficacy and adverse events in $16.3 \%$ of patients. By Kaplan-Meier 
Table 1 Patient demographics and characteristics.

\begin{tabular}{|c|c|}
\hline Total number of patients & 301 \\
\hline Mean age & $32.5(16.8)^{\mathrm{a}}$ \\
\hline Age range & $1-75$ \\
\hline Age distribution & $19.9 \%<18$ \\
\hline $\operatorname{Sex}(\mathrm{F} / \mathrm{M})$ & $156 / 145$ \\
\hline Weight $(\mathrm{kg})$ & $50.5(21.6)^{\mathrm{a}}$ \\
\hline \multicolumn{2}{|l|}{ Type of epilepsy } \\
\hline Localization-related & $217(72.1 \%)$ \\
\hline Generalized & $54(17.9 \%)$ \\
\hline Undetermined & $16(5.4 \%)$ \\
\hline Not classified & $14(4.7 \%)$ \\
\hline \multicolumn{2}{|l|}{ Seizure type } \\
\hline Partial onset & $243(80.7 \%)$ \\
\hline Generalized (including secondarily generalized) & $138(45.8 \%)$ \\
\hline Not classified & $19(6.3 \%)$ \\
\hline Nonepileptic seizures & $28(9.3 \%)$ \\
\hline Age at onset of seizures & $11.7(11.7)^{\mathrm{a}}$ \\
\hline Duration of epilepsy before drug introduction (years) & $21.3(12.9)^{\mathrm{a}}$ \\
\hline Number of concomitant AEDs & $2.16(0.827)^{\mathrm{a}}$ \\
\hline Number of mentally retarded patients & $98(32.6 \%)$ \\
\hline $\mathrm{CT}\left(+/-/\right.$ not reported, \%) ${ }^{\mathrm{b}}$ & $17.3 / 27.2 / 55.5$ \\
\hline MRI (+/-/not reported, \%) & $31.9 / 25.2 / 42.9$ \\
\hline
\end{tabular}

${ }^{\mathrm{a}}$ Mean (SD); ${ }^{\mathrm{b}}+$, abnormalities; -, no abnormalities.

Table 2 Reasons for discontinuing LEV over a follow-up period of 24 months.

\begin{tabular}{llll}
\hline & $\begin{array}{l}\text { Total study group } \\
(n=301)\end{array}$ & $\begin{array}{l}\text { Localization-related epilepsy } \\
(n=217)\end{array}$ & $\begin{array}{l}\text { Generalized epilepsy } \\
(n=54)\end{array}$ \\
\hline $\begin{array}{l}\text { Number of patients who } \\
\text { discontinued treatment }\end{array}$ & $138(45.8 \%)$ & $101(46.5 \%)$ & $25(46.3 \%)$ \\
& & & \\
Reason for discontinuation & & $37(17.1 \%)$ & $7(13.0 \%)$ \\
Lack of efficacy & $48(15.9 \%)$ & $12(5.5 \%)$ & $4(7.4 \%)$ \\
Adverse events & $18(6.0 \%)$ & $37(17.1 \%)$ & $8(14.8 \%)$ \\
Both & $49(16.3 \%)$ & $8(3.7 \%)$ & $5(9.3 \%)$ \\
Other & $23(7.7 \%)$ & &
\end{tabular}


survival analysis, the continuation rate was $76.0 \%$ after half a year, $65.6 \%$ after 1 year, $55.7 \%$ after 1.5 years, and $45.8 \%$ after 2 years (Figure $1 \mathrm{~A}$ ). Retention rates for patients with and without a mental handicap are illustrated in Figure 1B, which shows no difference in retention rate. The retention curves for patients with localizationrelated and generalized epilepsy are illustrated in Figure 1C, which indicates a lower retention rate for generalized epilepsy in the earlier phase, with a maximal difference at 67 days $(\mathrm{P}=0.005)$, and a pattern similar to that for localization-related epilepsy at a later stage. The mean length of LEV treatment for all patients was 392.2 (SD 235.7) days. The mean duration of drug treatment in patients who continued LEV was 516.7 days, compared with 243.8 days in the discontinuation group.

\section{Efficacy}

Seizure remission in patients on LEV is illustrated in Figure 2A. Between 12.9 and $16.7 \%$ of patients became seizure-free for a period of 3 months between baseline and 18 months of follow-up. An additional 10.6 to $36.0 \%$ of patients experienced a lower seizure frequency. Between 20.1 and $34.0 \%$ experienced no efficacy at all. About 10 to $15 \%$ reported a higher seizure frequency.

We have also investigated seizure remission from baseline up to all other assessment points (Figure $2 \mathrm{~B}$ ). About $14 \%$ of patients were seizure-free for half a year, and less than $10 \%$ for 1 year. Of all the patients who reached the last assessment point $(24$ months, $\mathrm{n}=10$ ), one patient was seizure-free from baseline.

Seizure freedom rates $(\sim 15 \%)$ only slightly differed at each assessment point between patients with localization-related epilepsy and patients with generalized epilepsy.

There are hardly any differences in discontinuation rates and reasons for discontinuation in both groups (Table 2).

Comparison of patients with and without a mental handicap indicates a different outcome (Table 3). Although a seizure reduction in $20-35 \%$ of patients with a mental handicap was noted at each assessment point, remission was attained by only a few patients. However, patients in this group do not more often discontinue LEV treatment than patients without a mental handicap. In addition, in the same number of patients, lack of efficacy was the main reason for discontinuation in both groups.

\section{Safety and tolerability}

The most frequently reported side effects per assessment point are listed in Table 4. We list only those adverse events affecting more than $5 \%$ of patients. The most prevalent adverse events in patients on LEV at almost each assessment point were activating mood disorders and tiredness. 

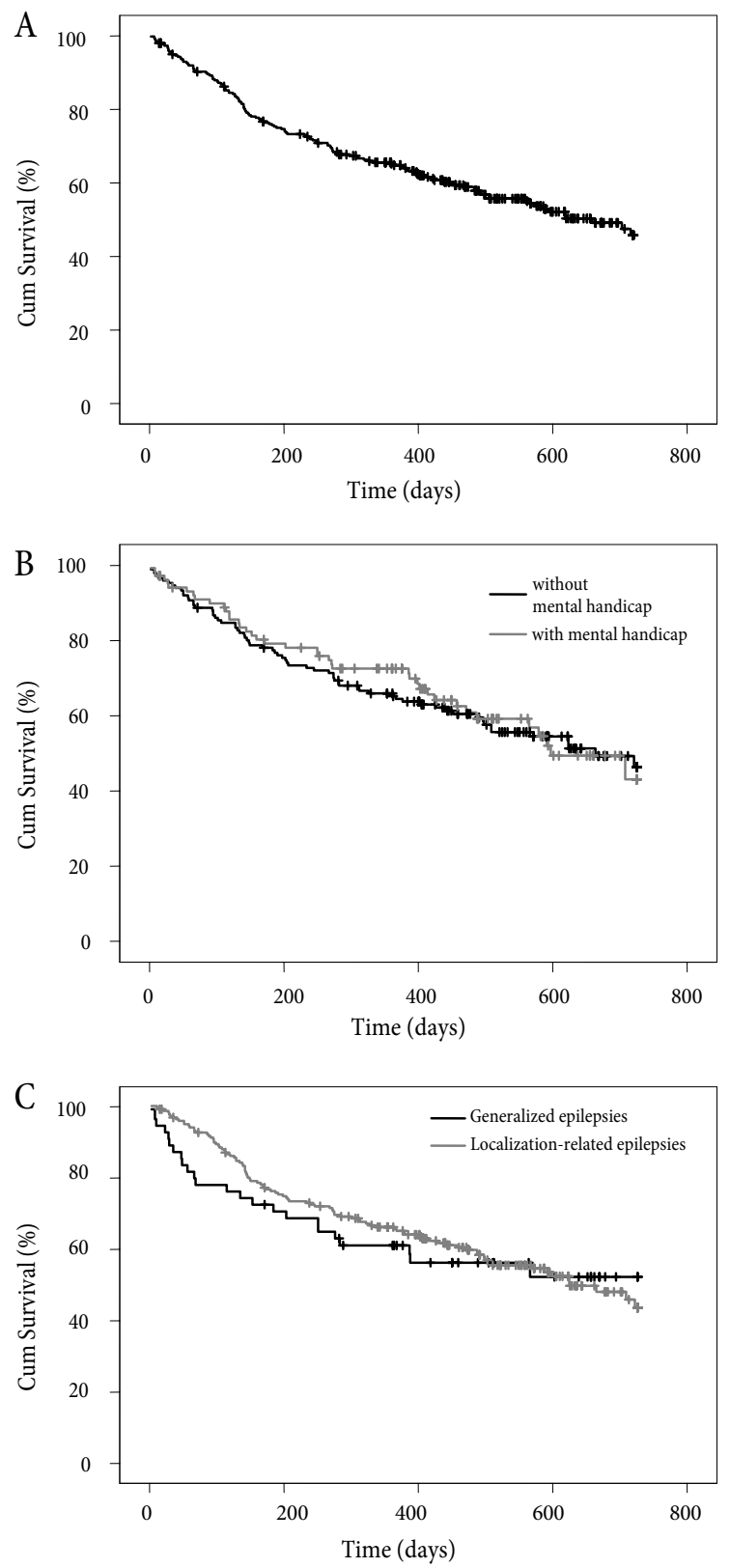

Figure 1 Kaplan-Meier survival curve illustrating the percentage of patients still being treated with LEV. (A) Total study group. (B) Comparison between patients with and without a mental handicap. (C) Comparison between patients with generalized epilepsy and those with localization-related epilepsy. 

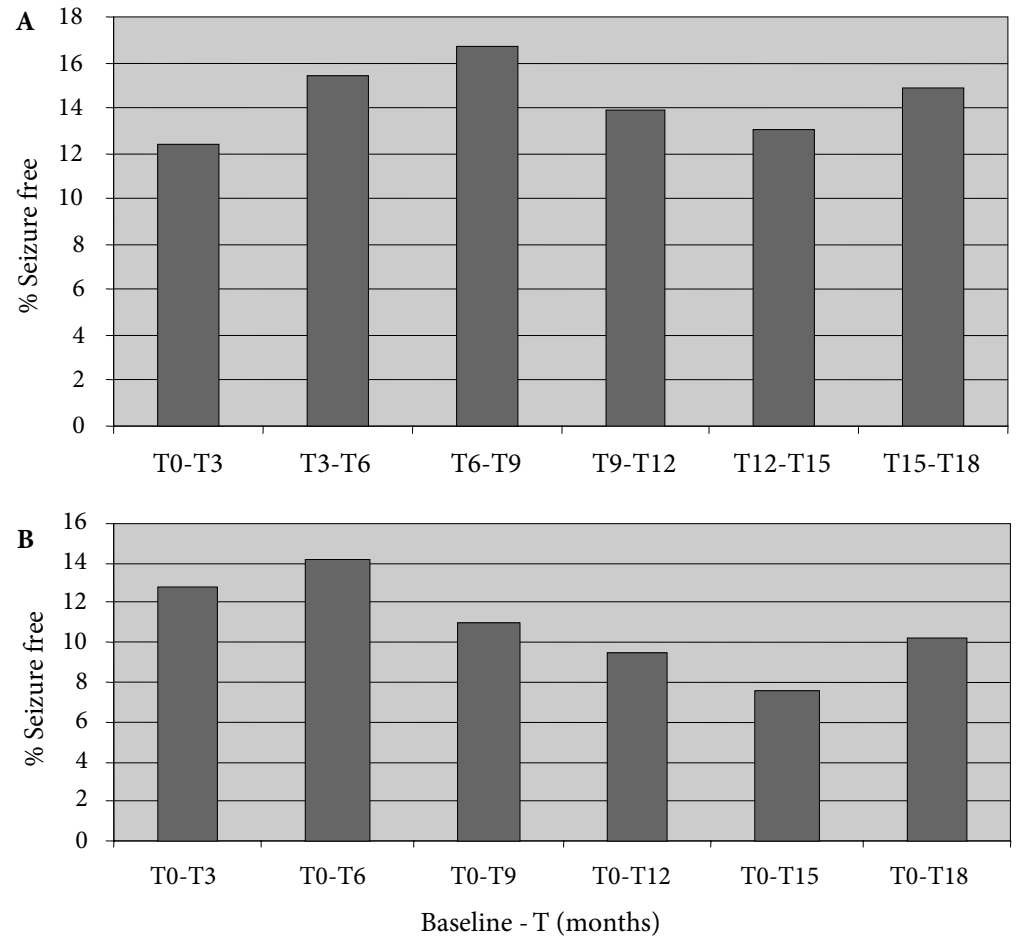

Figure 2 Seizure remission in patients being treated with LEV. (A) Seizure remission per 3-month interval. (B) Seizure remission from baseline up to all other assessment points.

A remarkable side effect of LEV was positive behavior, reported by about $7 \%$ of patients at 3, 6, 9, and 15 months of follow-up. Patients reported that they felt more energetic, active, and cheerful.

The most frequently reported side effects at the time of discontinuation were mood disorders-both activating (13.8\%) and deactivating (13.1\%)-tiredness (13.8\%), and sleepiness (8.5\%) (Table 5). Tiredness and sleeplessness were more frequently noted in patients without a mental handicap than in patients with a mental handicap. Behavioral adverse events, however, were more frequently reported in patients with a mental handicap (Table 3).

\section{Titration and dose}

Results with respect to titration and dose are listed in Table 6 . The mean starting dose at baseline was $617.5 \mathrm{mg} /$ day. The mean titration dose for LEV was $453.8 \mathrm{mg} /$ week. 
Table 3 Comparison between patients with and without a mental handicap.

\begin{tabular}{|c|c|c|}
\hline & $\begin{array}{l}\text { With mental } \\
\text { handicap }(n=98)\end{array}$ & $\begin{array}{l}\text { Without mental } \\
\text { handicap }(n=152)\end{array}$ \\
\hline $\begin{array}{l}\text { Number of patients who } \\
\text { discontinued treatment }\end{array}$ & $41(41.8 \%)$ & $70(46.1 \%)$ \\
\hline \multicolumn{3}{|l|}{ Reason for discontinuation } \\
\hline Lack of efficacy & $13(13.3 \%)$ & $24(15.8 \%)$ \\
\hline Adverse events & $5(5.1 \%)$ & $9(5.9 \%)$ \\
\hline Both & $12(12.2 \%)$ & $25(16.4 \%)$ \\
\hline Other & $5(5.1 \%)$ & $9(5.9 \%)$ \\
\hline \multicolumn{3}{|l|}{ Seizure freedom } \\
\hline 3 months & $3(5.5 \%)$ & $17(17.2 \%)$ \\
\hline 6 months & $1(2.0 \%)$ & $18(23.1 \%)$ \\
\hline 9 months & $3(8.3 \%)$ & $11(18.3 \%)$ \\
\hline 12 months & $2(5.0 \%)$ & $13(22.0 \%)$ \\
\hline 15 months & 0 & $6(14.6 \%)$ \\
\hline \multicolumn{3}{|c|}{ Side effects at discontinuation } \\
\hline Tiredness & 0 & $11(15.7 \%)$ \\
\hline Sleepiness & $4(9.8 \%)$ & $6(8.6 \%)$ \\
\hline Sleeplessness & 0 & $3(4.3 \%)$ \\
\hline \multicolumn{3}{|l|}{ Mood disorders } \\
\hline $\begin{array}{l}\text { Aggression, agitation, } \\
\text { hyperirritability }\end{array}$ & $8(19.5 \%)$ & $9(12.9 \%)$ \\
\hline Depression, apathy & $5(12.2 \%)$ & $6(8.6 \%)$ \\
\hline
\end{tabular}

Table 4 Most frequently reported side effects ${ }^{a}$.

\begin{tabular}{|c|c|c|}
\hline \multirow[t]{4}{*}{3 months } & Tiredness & $9.5 \%$ \\
\hline & Positive behavior ${ }^{\mathrm{b}}$ & $7.8 \%$ \\
\hline & Sleepiness & $5.6 \%$ \\
\hline & Mood disorders ${ }^{c}$ & $5.0 \%$ \\
\hline \multirow[t]{3}{*}{6 months } & Mood disorders ${ }^{c}$ & $8.1 \%$ \\
\hline & Tiredness & $7.4 \%$ \\
\hline & Positive behavior & $7.4 \%$ \\
\hline \multirow[t]{2}{*}{9 months } & Mood disorders ${ }^{c}$ & $7.3 \%$ \\
\hline & Positive behavior & $7.3 \%$ \\
\hline 12 months & Mood disorders ${ }^{c}$ & $5.2 \%$ \\
\hline 15 months & Positive behavior & $6.3 \%$ \\
\hline \multirow[t]{2}{*}{18 months } & Mood disorders ${ }^{\mathfrak{c}}$ & $10.6 \%$ \\
\hline & Tiredness & $6.4 \%$ \\
\hline
\end{tabular}

a Only side effects that occurred in $>5 \%$ of the patients are reported; ${ }^{\mathrm{b}}$ Positive behavior: being in a better condition; taking more initiatives; being more calm, active, cheerful, etc.; ${ }^{\mathrm{c}}$ Agitation, aggression, hyperirritability. 
Table 5 Side effects reported by patients who discontinued drug treatment over a follow-up period of 24 months.

\begin{tabular}{lc}
\hline Agitation, aggression, hyperirritability & $18(13.8 \%)$ \\
Tiredness & $18(13.8 \%)$ \\
Depression, apathy & $17(13.1 \%)$ \\
Sleepiness & $11(8.5 \%)$ \\
Skin complaints & $6(4.6 \%)$ \\
Asthenia & $5(3.8 \%)$ \\
Headache & $5(3.8 \%)$ \\
Endocrine complaints & $5(3.8 \%)$ \\
Sleeplessness & $4(3.1 \%)$ \\
Dizziness & $4(3.1 \%)$ \\
Ataxia & $4(3.1 \%)$ \\
Gastrointestinal complaints & $4(3.1 \%)$ \\
Double vision & $2(1.5 \%)$ \\
Cardiovascular complaints & $2(1.5 \%)$ \\
Other CNS complaints & $2(1.5 \%)$ \\
Other non-CNS complaints & $2(1.5 \%)$ \\
Eye complaints & $1(0.8 \%)$ \\
\hline
\end{tabular}

Table 6 Levetiracetam titration schedules and dosages.

\begin{tabular}{lcl}
\hline Titration schedule & \multicolumn{2}{l}{ Frequency $^{\mathrm{a}}$} \\
\hline Individual schedule & 211 & $(70.1 \%)$ \\
$500 \mathrm{mg} /$ day/2 weeks & 25 & $(8.3 \%)$ \\
$500 \mathrm{mg} /$ day/4 weeks & 0 & \\
$1000 \mathrm{mg} /$ day/2 weeks & $44 \quad(14.6 \%)$ \\
$1000 \mathrm{mg} /$ day/4 weeks & 2 & $(0.7 \%)$ \\
Starting dose (mg/day) & $617.5(299.6)^{\mathrm{b}}$ \\
Titration dose (mg/week) & $453.8(195.6)^{\mathrm{b}}$ \\
Dose of patients who discontinued treatment because of & \\
$\quad$ Lack of efficacy & $2281.3(789.9)^{\mathrm{b}}$ \\
Side effects & $1500.0(870.3)^{\mathrm{b}}$ \\
Dose of patients in remission & $1970.6(659.4)^{\mathrm{b}}$ \\
\hline
\end{tabular}

${ }^{\mathrm{a}}$ Number (\%) of patients; ${ }^{\mathrm{b}}$ Mean (SD). 
Table 7 Comparison between patients discontinuing and those continuing LEV at 12 months.

\begin{tabular}{|c|c|c|c|c|c|}
\hline & \multicolumn{2}{|c|}{$\begin{array}{l}\text { Patients who discontinued } \\
\text { treatment } \\
(n=103)\end{array}$} & \multicolumn{2}{|c|}{$\begin{array}{l}\text { Patients who continued } \\
\text { treatment } \\
(n=168)\end{array}$} & \multirow[t]{2}{*}{$P$} \\
\hline \multicolumn{5}{|l|}{ Variables predicting (dis)continuation } & \\
\hline Age & $34.3(17.7)^{\mathrm{a}}$ & & $31.5(16.1)^{\mathrm{a}}$ & & 0.191 \\
\hline $\operatorname{Sex}(F / M)$ & $55 / 48$ & & $87 / 81$ & & 0.796 \\
\hline Weight & $51.6(16.6)^{\mathrm{a}}$ & & $49.6(23.2)^{\mathrm{a}}$ & & 0.972 \\
\hline Age at onset of seizures & $12.7(11.3)^{\mathrm{a}}$ & & $11.7(11.8)^{\mathrm{a}}$ & & 0.572 \\
\hline $\begin{array}{l}\text { Duration of epilepsy before drug } \\
\text { introduction (years) }\end{array}$ & $22.4(14.1)^{\mathrm{a}}$ & & $20.5(12.1)^{\mathrm{a}}$ & & 0.268 \\
\hline Number of mentally retarded patients & $25(24.3 \%)$ & & $57(33.9 \%)$ & & 0.093 \\
\hline CT abnormalities & $21(20.4 \%)$ & & $24(14.3 \%)$ & & 0.190 \\
\hline MRI abnormalities & $40(38.8 \%)$ & & $49(29.2 \%)$ & & 0.100 \\
\hline \multicolumn{6}{|l|}{ Type of epilepsy } \\
\hline Localization-related & $74(71.8 \%)$ & & $123(73.2 \%)$ & & 0.806 \\
\hline Generalized & $21(20.4 \%)$ & & $26(15.5 \%)$ & & 0.300 \\
\hline \multicolumn{6}{|l|}{ Seizure type } \\
\hline Partial onset & $87(84.5 \%)$ & & $135(80.4 \%)$ & & 0.394 \\
\hline $\begin{array}{l}\text { Generalized } \\
\text { (including secondarily generalized) }\end{array}$ & $46(44.7 \%)$ & & $75(44.6 \%)$ & & 0.998 \\
\hline $\begin{array}{l}\text { Number of AEDs started and stopped } \\
\text { before drug introduction }\end{array}$ & $4.37(2.1)^{\mathrm{a}}$ & & $4.52(\mathrm{SD} 2.4)$ & & 0.534 \\
\hline Starting dose (mg/day) & $599.5(274.2)^{\mathrm{a}}$ & & $623.5(289.5)^{\mathrm{a}}$ & & 0.500 \\
\hline Titration dose (mg/week) & $438.9(193.1)^{\mathrm{a}}$ & & $457.7(197.7)^{\mathrm{a}}$ & & 0.475 \\
\hline Dose (mg/day) & $1951.5(898.0)^{\mathrm{a}}$ & & $1988.9(843.0)^{\mathrm{a}}$ & & 0.725 \\
\hline $\begin{array}{l}\text { Patients with individual titration } \\
\text { schedule (\%) }\end{array}$ & 76.3 & & 73.2 & & 0.626 \\
\hline Mean drug load of other AEDs & 1.73 & & 1.73 & & \\
\hline \multicolumn{6}{|c|}{ Mean $[S D]$ dose (mg/day) of comedication (\% of patients) } \\
\hline Diamox & $187.5[88.4]$ & $(1.9 \%)$ & $535.0[267.9]$ & $(4.9 \%)$ & \\
\hline $\mathrm{CBZ}^{\mathrm{b}}$ & $944.4[479.0]$ & $(35.0 \%)$ & $804.0[335.4]$ & $(36.9 \%)$ & \\
\hline CLB & $19.9[18.1]$ & $(35.9 \%)$ & $20.4[15.9]$ & $(33.0 \%)$ & \\
\hline CLN & $4.5[8.2]$ & $(6.8 \%)$ & $2.3[1.6]$ & $(4.9 \%)$ & \\
\hline ETS & $729.3 \quad[236.4]$ & $(2.9 \%)$ & $791.7 \quad[260.2]$ & $(2.9 \%)$ & \\
\hline FBM & 1200.0 & $(1.0 \%)$ & - & & \\
\hline PHB & - & & $125.0 \quad[35.4]$ & $(1.9 \%)$ & \\
\hline PHT & 309.9 [111.6] & $(13.6 \%)$ & $338.2 \quad[99.2]$ & $(19.4 \%)$ & \\
\hline GBP & 600.0 & $(1.0 \%)$ & - & & \\
\hline LMT & $329.3[241.1]$ & $(35.0 \%)$ & $379.1 \quad[210.3]$ & $(35.0 \%)$ & \\
\hline OXC & $1340.0[499.0]$ & $(14.6 \%)$ & 1457.1 [400.9] & $(13.6 \%)$ & \\
\hline TPM & 321.9 [276.3] & $(7.8 \%)$ & $112.5[123.7]$ & $(1.9 \%)$ & \\
\hline VPA & $1472.2[945.1]$ & $(17.5 \%)$ & $1206.7[742.4]$ & $(14.6 \%)$ & \\
\hline VGB & 3000.0 & $(1.0 \%)$ & - & & \\
\hline
\end{tabular}




\begin{tabular}{|c|c|c|}
\hline & $\begin{array}{l}\text { Patients who discontinued } \\
\text { treatment } \\
(n=103)\end{array}$ & $\begin{array}{l}\text { Patients who continued } \\
\text { treatment } \\
(n=168)\end{array}$ \\
\hline \multicolumn{3}{|l|}{ Seizure reduction } \\
\hline Seizure free & $1(1.0 \%)$ & $13(7.7 \%)$ \\
\hline Lower seizure frequency & $4(3.9 \%)$ & $15(8.9 \%)$ \\
\hline No efficacy & $58 \quad(56.3 \%)$ & $27(16.1 \%)$ \\
\hline Higher seizure frequency & $25(24.3 \%)$ & $19(11.3 \%)$ \\
\hline Change not reported & $15(14.6 \%)$ & $94(56.0 \%)$ \\
\hline \multicolumn{3}{|l|}{ Side effects } \\
\hline \multicolumn{3}{|l|}{ Mood disorders } \\
\hline $\begin{array}{l}\text { Aggression, agitation, } \\
\text { hyperirritability }\end{array}$ & $12(11.7 \%)$ & $4(2.4 \%)$ \\
\hline Depression, apathy & $14(13.6 \%)$ & $1(0.6 \%)$ \\
\hline Tiredness & $16(15.5 \%)$ & $4(2.4 \%)$ \\
\hline Sleepiness & $11(10.7 \%)$ & $3(1.8 \%)$ \\
\hline
\end{tabular}

Very soon after baseline, a dose between 2000 and $2500 \mathrm{mg}$ /day was reached, with continuation of this dose until 24 months after baseline. The highest dose achieved was $4000 \mathrm{mg} /$ day.

We have also analyzed the different titration schedules for LEV as recommended by the manufacturer, as these schedules are a subject of frequent discussion among clinicians. The most frequent fixed titration schedule was $1000 \mathrm{mg} /$ day/2 weeks (14.6\%). The most frequently applied strategy was an individual approach (70.1\%), which means a faster titration compared with the recommendations of the manufacturer. The most cautious recommended titration schedule is not applied. Patients who discontinued treatment because of side effects reached a dose of $1500 \mathrm{mg} /$ day instead of the $2281 \mathrm{mg} /$ day reached by patients who discontinued because of a lack of efficacy. This is remarkable because the manufacturer recommends doses up to 3000 $\mathrm{mg}$ /day. Only a quarter of all patients at each assessment point reached that dose. The mean dose for patients who became seizure-free was less than $2000 \mathrm{mg} / \mathrm{day}$.

\section{Variables predicting (dis)continuation}

From the beginning of 2001 to the final assessment point, 138 patients discontinued LEV treatment and 163 patients continued treatment. We subsequently analyzed 
whether differences existed between those who discontinued the drug between baseline and the first 12 months of treatment and those who continued LEV treatment. Our analysis considered only those patients who where able to reach this 12-month assessment point $(\mathrm{n}=271)$ (Table 7$)$. This analysis indicates only small differences with respect to demographic variables. Results with respect to titration and dose are the same in both groups. Even the mean drug load of AEDs other than LEV and the percentages of patients on other AEDs do not differ. Thus, no variables predicting (dis)continuation can be identified. Only major differences were seen in the outcome variables seizure reduction and side effects.

\section{I S C USS I O N}

LEV was recently introduced into clinical practice with a potentially favorable efficacy and tolerability profile and a unique mechanism of action. Clinical experience with this drug has been reported in some studies. The added value of this study is due to the large study population ( $\mathrm{n}=301$ ), the long follow-up period (up to 24 months), and the low risk of selection bias by inclusion of all patients who received LEV in the period 2001-2003 in a tertiary epilepsy center. This is illustrated by the characteristics of our study population, specifically by the wide age range and large numbers of children and mentally retarded individuals. In total, 138 of 301 patients (45.8\%) stopped LEV treatment at some point during a follow-up period of 2 years. Our study recorded retention rates of $65.6 \%$ after 1 year and $45.8 \%$ after 2 years using KaplanMeier survival analysis. Previous studies have reported higher continuation rates of $74 \%$ after 1 year and $58 \%$ after 2 years ${ }^{10}$. Depondt et al. reported a retention rate of $58 \%$ after 3 years ${ }^{11}$. It is not clear whether this variation is caused by differences in study populations. However, another study including a large number of mentally retarded patients reported a similar retention rate after 1 year ${ }^{15}$. Compared with other new AEDs, LEV seems to have a superior retention rate, considering the 1-year retention rates of $55 \%$ for topiramate ${ }^{16}, 60 \%$ for lamotrigine ${ }^{17}, 58 \%$ for vigabatrin ${ }^{17}$, and $45 \%$ for gabapentin ${ }^{17}$. Nevertheless, it is disappointing to see that after 2 years, more than half of the patients who started with LEV have already discontinued treatment. The Kaplan- Meier curve illustrates that the largest percentage of withdrawals occurred in the first period of treatment up to 6 months. After 6 months, the number of withdrawals more or less remained steady at a lower rate.

The main reason for discontinuation was lack of efficacy. In one-third of the total study group on LEV, lack of efficacy played a role in discontinuation of treatment. When only those patients who discontinued treatment are considered, lack of efficacy is the only or one of the reasons for $70 \%$ of the discontinuations. Discontinuation due to adverse events only was reported in a mere $6 \%$ of patients. In line with our study, 
results of other postmarketing studies indicate that lack of efficacy led more often to discontinuation of treatment than adverse events ${ }^{18-20}$.

Compared with other new AEDs, the effects of LEV on seizure frequency are promising. About $15 \%$ of patients in this highly refractory group have a 3-month remission, whereas $10 \%$ of patients become seizure-free for longer periods. The numbers of patients in remission are comparable to long-term data from RCTs, with $10.2 \%$ seizure free for at least 6 months, compared to $13 \%$ seizure remission in regulatory clinical trials (RCTs) ${ }^{20}$.

Our study shows that LEV is efficacious in patients with localization-related epilepsy as well as in patients with generalized epilepsy. A comparable seizure remission of about $15 \%$ of patients is achieved at almost every assessment point for both types of epilepsy. Retention curves also indicate similar patterns. Other studies have already demonstrated that LEV appears to be useful add-on treatment in patients with resistant generalized epilepsy, with the achievement of similar remission rates $^{21,22}$. Thus, evidence is expanding that LEV may be used in clinical practice as a broad-spectrum AED.

The percentages of patients with a mental handicap achieving seizure remission is, however, low. Nevertheless, retention rates for patients with and without a mental handicap are the same. With similar discontinuation rates (due to lack of efficacy or adverse events) and with the assumption that LEV is far less efficacious in patients with a learning disability, there must be another reason why patients in this group continue treatment. One explanation may be that these patients experienced a positive effect on their behavior and therefore did not discontinue treatment, although LEV was not efficacious in terms of achieving seizure remission. Only one study has reported the occurrence of positive behavior in patients with epilepsy receiving $\mathrm{LEV}^{19}$. Accordingly, LEV has been found to reduce hyperactivity, impulsivity, and aggression in an open study in a limited number of autistic children without epilepsy ${ }^{23}$.

In our study, activating mood disorders, tiredness, and positive behavior are the most frequently reported side effects per assessment point. Clinically of greater importance are the reported side effects in patients who discontinued treatment. Mood disorders, activating and deactivating, are (one of) the most important side effects leading to discontinuation of drug treatment, implying that activating mood disorders are better tolerated than deactivating mood disorders, which seem to lead to immediate discontinuation of LEV treatment. This is remarkable because LEV is characterized as an activating drug in the literature ${ }^{24}$. RCTs indicate that only somnolence, asthenia, dizziness, headache, and infection are major adverse events. However, as already mentioned, our study demonstrates that mood disorders are the most important side effect, reported in more than $26 \%$ of patients who discontinued treatment. In line with our study, White et al. reported that behavioral side effects were the most common reason for drug discontinuation ${ }^{25}$. These results highlight 
the need for clinical studies in addition to RCTs. Mood disorders were also the most frequently reported adverse event in patients with a mental handicap, whereas tiredness and sleeplessness were not noted at all in this group. Of course, this may reflect report bias. However, sleep behavior was not scored on the basis of self-reports but by observation of nursing staff, similar to the behavioral adverse events.

Easy titration with LEV is illustrated by data indicating that the most cautious titration schedule was not applied in a single patient and that neurologists often chose a faster titration schedule than recommended by the manufacturer. Among the individual schedules, by far the most frequently applied was $500 \mathrm{mg} /$ day/week.

The mean starting dose was lower than those applied in some clinical trials ${ }^{4-6}$, in which $1000 \mathrm{mg} /$ day (in some patient groups) was a frequently chosen starting dose. The clinical dose achieved in our population was between 2000 and $2500 \mathrm{mg} /$ day (adults only), and this dose was reached within a few weeks. Nevertheless, the maximum dose of $3000 \mathrm{mg} /$ day as recommended by the manufacturer was achieved in only a minority of patients. Explanations may be the achievement of seizure remission on lower dosages or the development of side effects: the mean dose for patients who became seizure-free was less than $2000 \mathrm{mg} /$ day, and patients who discontinued treatment due to adverse events did not reach doses higher than 1500 $\mathrm{mg}$ /day. However, in some cases, higher dosages seem feasible, as illustrated by data indicating that the mean dose for patients who discontinued treatment because of lack of efficacy was $2281 \mathrm{mg} /$ day. It is not clear why neurologists did not try to reach higher dosages. However, pooled data from the three pivotal trials indicate that there is hardly a difference in responder rates between 2000 and $3000 \mathrm{mg} / \mathrm{day}^{26}$.

An important aspect of our evaluation was analysis of differences between patients who continued and patients who discontinued treatment. Such a comparison could provide factors prognostic of LEV failure/success that could be used in clinical decision making. A high maximum dose, a low starting dose, the presence of generalized seizures, and a smaller number of AEDs at baseline have been reported as positive prognostic variables for continuation in a previous study ${ }^{20}$. However, no variables predicting (dis)continuation could be identified in our study. Obviously, most clinical factors are not useful in clinical decision making.

\section{ONCLUSION}

LEV appears to be a useful broad-spectrum AED. Its side effect profile is mild, with mood disorders being the most important adverse event. Even in patients who are mentally retarded, treatment with LEV may be valuable, although the drug is not efficacious in terms of seizure remission in this group of patients. 


\section{R E FER E NCES}

1. Stefan H, Krämer G, Mamoli B, editors. Challenge epilepsy: new antiepileptic drugs. Berlin: Blackwell Science; 1998.

2. Lynch BA, Lambeng N, Nocka K, et al. The synaptic vesicle protein SV2A is the binding site for the antiepileptic drug levetiracetam. Proc Natl Acad Sci USA 2004;101:9861-6.

3. Patsalos PN. Pharmacokinetic profile of levetiracetam: toward ideal characteristics. Pharmacol Ther 2000;85:77-85.

4. Cereghino JJ, Biton V, Abou-Khalil B, Dreifuss F, Gauer LJ, Leppik I. Levetiracetam for partial seizures: results of a double-blind, randomized clinical trial. Neurology 2000;55:236-42.

5. Ben-Menachem E, Falter U for the European Levetiracetam Study Group. Efficacy and tolerability of levetiracetam $3000 \mathrm{mg} / \mathrm{d}$ in patients with refractory partial seizures: a multicenter, double-blind, responder-selector study evaluating monotherapy. Epilepsia 2000;41:1276-83.

6. Shorvon SD, Lowenthal A, Janz D, Bielen R, Loiseau P. For the European Levetiracetam Study Group. Multicenter double-blind, randomized placebo-controlled trial of levetiracetam as add-on ther apy in patients with refractory partial seizures. Epilepsia 2000;41:1179-86.

7. Walker MC, Sander JWAS. Difficulties in extrapolating from clinical trial data to clinical practice: the case of antiepileptic drugs. Neurology 1997;49:333-7.

8. Walker MC, Sander JWAS. Topiramate. Seizure 1996;5:199-204.

9. Walker MC, Sander JW. The impact of new antiepileptic drugs on the prognosis of epilepsy: seizure freedom should be the ultimate goal. Neurology 1996;46:912-4.

10. Nicolson A, Lewis SA, Smith DF. A prospective analysis of the outcome of levetiracetam in clinical practice. Neurology 2004;63:568-70.

11. Depondt C, Yuen AWC, Bell GS, et al. The long term retention of levetiracetam in a large cohort of patients with epilepsy. J Neurol Neurosurg Psychiatry 2006;77:101-3.

12. Ketter TA, Post RM, Theodore WH. Positive and negative psychiatric effects of antiepileptic drugs in patients with seizure disorders. Neurology 1999;53(Suppl. 2):S53-67.

13. Roberts GMP, Majoie HJM, Leenen LAM, et al. Ketter's hypothesis of the mood effects of antiepileptic drugs coupled to the mechanism of action of topiramate and levetiracetam. Epilepsy Behav 2005;6:366-72.

14. Kaplan EL. Non-parametric estimation from incomplete observations. Am Stat Assoc J 1958;53:457-81.

15. Ben-Menachem E, Gilland E. Efficacy and tolerability of levetiracetam during 1-year follow-up in patients with refractory epilepsy. Seizure 2003;12:131-5.

16. Ketter MW, Smith DF, Stockton PA, Chadwick DW. Topiramate in clinical practice: first year's postlicensing experience in a specialist epilepsy clinic. J Neurol Neurosurg Psychiatry 1999;66:759-63.

17. Wong ICK. New antiepileptic drugs: study suggests that a quarter of patients will still be taking the new drugs after six years. Br Med J 1997;314:603-4.

18. Abou-Khalil B, Schaich L. Long-term efficacy of levetiracetam for partial seizures. Seizure 2005; 14:577-85. 
19. Brodtkorb E, Klees TM, Nakken KO, Lossius R, Johannessen SI. Levetiracetam in adult patients with and without learning disability: focus on behavioral adverse effects. Epilepsy Behav 2004;5:231-5.

20. Krakow K, Walker M, Otoul C, Sander JWAS. Long-term continuation of levetiracetam in patients with refractory epilepsy. Neurology 2001;56:1772-4.

21. Kumar SP, Smith PEM. Levetiracetam as add-on therapy in generalised epilepsies. Seizure 2004;13:475-7.

22. Betts T, Waegemans T, Crawford P. A multicentre, double-blind randomized, parallel group study to evaluate the tolerability and efficacy of two oral doses of levetiracetam, $2000 \mathrm{mg}$ daily and $4000 \mathrm{mg}$ daily, without titration in patients with refractory epilepsy. Seizure 2000;9:80-7.

23. Rugino TA, Samsock TC. Levetiracetam in autistic children: an open label study. J Dev Behav Pediatr 2002;4:225-30.

24. Reijs R, Aldenkamp AP, De Krom M. Mood effects of antiepileptic drugs. Epilepsy Behav 2004;5(Suppl. 1):S66-76.

25. White JR, Walczak TS, Leppik LE, et al. Discontinuation of levetiracetam because of behavioral side effects: a case-control study. Neurology 2003;61:1218-21.

26. Privitera M. Efficacy of levetiracetam: a review of three pivotal clinical trials. Epilepsia 2001;42(Suppl. 4):31-5. 


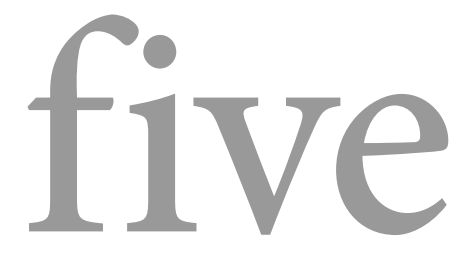

\section{Lamotrigine in clinical practice: long-term experience in patients with refractory epilepsy referred to a tertiary epilepsy center}




\section{A B S T R A C T}

Lamotrigine (LTG, Lamictal), one of the newer antiepileptic drugs, was admitted to the Dutch market in 1996. It was first used as adjunctive therapy and later as a monotherapy in partial and generalized epilepsy. All patients who started on LTG in 1996 or 1997 in the Epilepsy Centre Kempenhaeghe $(n=314)$ were enrolled in this study and followed for 48 months. The data indicate that the retention rates for LTG after $1,2,3$, and 4 years are respectively $74.4,69.3,63.1$, and 55.6\%. Patients with normal cognitive function were more likely to continue than patients with mental retardation. The main reason for discontinuing LTG therapy was lack of efficacy (19.1\%).

Four patients (1.4\%) were seizure-free for the total follow-up period of 48 months. The most frequently reported negative side effects were dizziness and headache, both in patients who continued and in those who discontinued therapy. A large percentage of patients also reported positive side effects like "feeling/being more active" and "feeling more clear/more responsive." For the whole patient group, the plasma level of LTG was measured 277 times. Plasma levels of LTG were influenced by the patients' comedications. Plasma levels of LTG in groups taking LTG in monotherapy, LTG plus an inducer, and LTG plus valproate were $8.7,4.8$, and $8.7 \mathrm{mg} / \mathrm{L}$, respectively.

The correlation between measured plasma level and dose confirm the manufacturer's dose recommendations. The manufacturer recommends half the dosage of lamotrigine monotherapy when the patient also uses valproate. When the patient uses an inducer, the dosage of LTG must be two times the dose used in monotherapy. 


\section{N T RODUCT IO N}

Lamotrigine (LTG, Lamictal), one of the newer antiepileptic drugs (AEDs), was admitted to the Dutch market in 1996. It was first used as adjunctive therapy and later as monotherapy in partial and generalized epilepsy. LTG is also used to treat patients with Lennox-Gastaut syndrome and other childhood epilepsies ${ }^{1}$.

This study is part of a series of studies on new antiepileptic drugs in our own tertiary epilepsy referral and care center, Kempenhaeghe. Information on the use (retention rate), efficacy, side effects, and plasma levels of LTG was collected for all patients who started LTG within a specific period. Topiramate and levetiracetam have been covered in previous studies in this series ${ }^{2,3}$.

The retention rate (RR) of a drug can be used as a predictor of the efficacy and tolerability of that drug. In the case of serious side effects or lack of efficacy, patients usually discontinue the treatment, leading to lower $\mathrm{RRs}^{4-7}$.

There is a linear relationship between plasma LTG concentration and dosage, but there is considerable interindividual variability ${ }^{8}$. This is one of the reasons why therapeutic drug monitoring (TDM) may be useful in LTG therapy ${ }^{8}$. Because patients have seizures at irregular intervals, and some patients have infrequent seizures, it can be difficult to select a dose based on titration to effective seizure control. In such patients, it may be useful to select a reasonable initial target serum level. Furthermore, clinical signs and symptoms of toxicity may be difficult to detect and interpret, particularly in patients with intellectual disabilities who may not be able to report symptoms. In these patients, TDM can also be useful ${ }^{9}$.

Plasma levels of LTG measured in the first phase 2 and phase 3 studies indicated an average plasma level of $1-3 \mathrm{mg} / \mathrm{L}^{10-16}$. When $\mathrm{LTG}$ was used in clinical practice, patients appeared to tolerate higher plasma levels, sometimes with greater efficacy. According to Hirsch et al. ${ }^{17}$, plasma levels of LTG $<10 \mathrm{mg} / \mathrm{L}$ rarely result in toxic reactions. Morris et al. ${ }^{18,19}$ and others ${ }^{20-22}$ advise a plasma LTG level of 3-14 mg/L.

\section{METHOD}

This study was approved by the medical ethics committee. All patients who started on LTG in 1996 and 1997 in the Epilepsy Centre Kempenhaeghe were selected using the automated medical information system (MIS).

A database was created in Microsoft Access to contain the information on the selected patients. Parameters in the database included patient characteristics (age, sex, weight), age at onset of seizures, mental retardation (IQ $<70^{9}$ ), CT/MRI abnormalities, history of drug use, titration schedule for LTG, efficacy, start and possible stop dates, reported side effects, and reasons for discontinuation of LTG. Reasons for 
discontinuation were entered into the database using the following five identifiers: lack of efficacy, adverse events, both, other, and unknown. Type of epilepsy and types of seizures were classified using the International League Against Epilepsy (ILAE) classification. All data were acquired from the MIS and the written individual patient files.

All selected patients were followed at 6-month intervals for 48 months. Every 6 months, side effects and efficacy were evaluated. Efficacy of LTG was entered into the database using a 4-point scale consisting of: seizure-free for the 6 months, lower seizure frequency, no efficacy, higher seizure frequency, and not reported. All measured plasma LTG levels were acquired from the MIS.

Statistical analysis was performed using SPSS 14.0 for Windows. Descriptive statistics were calculated for each point. The RR of LTG was calculated using KaplanMeier survival analysis. Other data were evaluated using an independent-sample $t$ test, the Mann-Whitney U test, or the Pearson $\chi^{2}$ test.

\section{R ES U L T S}

A total of 392 patients were identified who started LTG treatment in 1996 and 1997. In total, 78 patients (19.9\%) were excluded. There were three different reasons for exclusion: the patient had used LTG before referral to our center $(12.8 \%)$, data were missing (4.1\%), and the patient died before the end of the study (3.1\%). Many analyses were performed on the remaining 314 patients. Analysis of RR was performed on a smaller group, that is, excluding patients lost to follow-up before the end of the 4-year follow-up period $(\mathrm{n}=293)$.

Table 1 lists the main characteristics of the patients. The mean age of the study group was 32.1 years (SD 16.3). Patients had had epilepsy for a mean of 21.3 (SD 14.2) years before LTG introduction. Most patients had localization-related epilepsy; generalized seizures were the most frequent seizure type.

RRs were calculated using Kaplan-Meier survival analysis. All patients were included in this analysis (Figure 1).

Of 293 patients, 130 (44.4\%) discontinued LTG treatment over the 4 years (RR = $56.6 \%)$. The RR for patients with mental retardation was $50 \%$ after 4 years. Patients with normal cognitive function were more likely to continue ( $42 \%$ discontinued, RR $=58 \%)$. The RRs of the total group after 1,2, 3, and 4 years are listed in Table 2 .

Most patients discontinued LTG because of lack of efficacy (19.1\%); 12.3\% of the patients stopped because of adverse effects (Table 3). Mentally retarded patients did not differ from other patients with respect to reasons for discontinuing LTG.

Percentages of seizure reduction in four classes in patients who continued LTG are listed in Table 4. 
Table 1 Patient demographics and characteristics.

\begin{tabular}{ll}
\hline Total no. of patients & 314 \\
Age & $32.1(16.3)^{\mathrm{a}}$ \\
Age range & $1-74$ \\
Age distribution & $<18$ years old: $\mathrm{n}=66(21 \%)$ \\
Age at onset of seizures & $11.1(11.2)^{\mathrm{a}}$ \\
Duration of epilepsy before LTG introduction (years) & $21.3(14.2)^{\mathrm{a}}$ \\
Sex (M/F) & $153 / 161(48.7 / 51.3 \%)$ \\
Weight (kg) & $63.8(20.4)^{\mathrm{a}}$ \\
& \\
Type of epilepsy & \\
Localization-related & $218(69.4 \%)$ \\
Generalized & $57(18.2 \%)$ \\
Undetermined whether focal or generalized & $6(1.9 \%)$ \\
Special syndromes & $1(0.3 \%)$ \\
& \\
Seizure type & \\
Partial seizures & \\
Generalized (including secondarily) & $303(96 \%)$ \\
Unclassified & $340(108.3 \%)$ \\
Other seizure types & $22(7.0 \%)$ \\
Mentally retarded (yes/no/unknown) & $40(12.7 \%)$ \\
CT (yes/no/unknown) & \\
MRI (yes/no/unknown) & $101 / 177 / 36(32.2 / 56.4 / 11.5 \%)$ \\
No. of concomitant AEDs at baseline including LTG & $62 / 117 / 135(19.7 / 37.3 / 43.0 \%)$ \\
Total drugload at baseline including LTG & $82 / 68 / 164(26.1 / 21.7 / 52.2 \%)$ \\
\hline
\end{tabular}

${ }^{\mathrm{a}}$ Mean (SD); ${ }^{\mathrm{b}}$ ILAE classification; ${ }^{\mathrm{c}}$ Range.

Seizure reduction in patients who discontinued LTG is outlined in Table 5. Most of the patients who discontinued during the first 6 months experienced an increase in seizure frequency (42.3\%). Most patients who discontinued LTG therapy after 12 or 24 months had no change in seizure frequency or had an increase in seizure frequency.

Thirty (10.2\%) patients were seizure-free after 6 months of LTG treatment. After 2 years of follow-up, 5 remained seizure-free. Four patients remained seizure-free for the full 4-year follow-up period (Table 6). All these patients had generalized epilepsy. Three of the four patients had generalized seizures. Three of these four patients used LTG in combination with valproate.

The most frequently reported side effects in the total group $(\mathrm{n}=314)$, spontaneously reported by patients, are listed in Table 7. A large percentage of patients experienced dizziness at each interval. The side effect "more active" can be considered a 


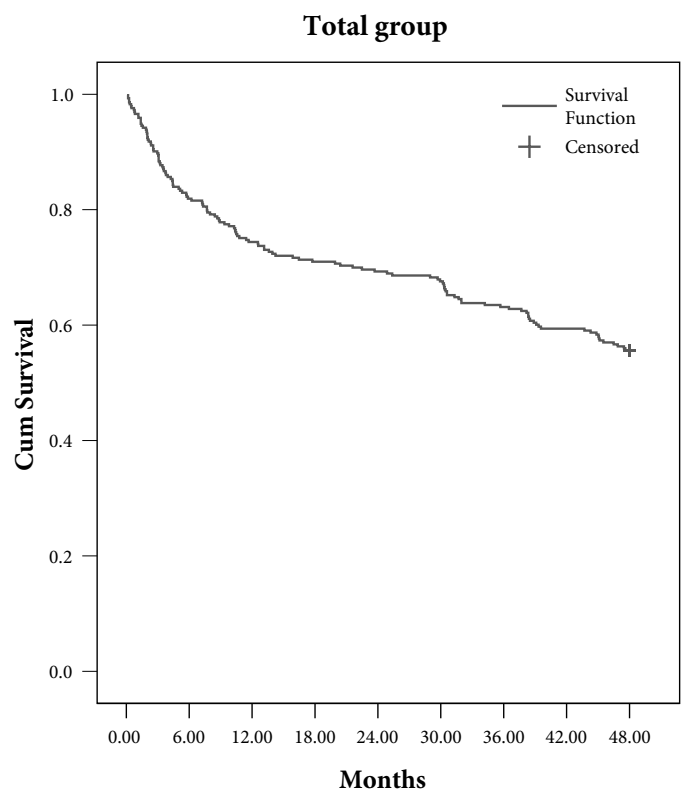

Figure 1 Kaplan-Meier survival analysis.

Table 2 RRs of LTG after 1, 2, 3, and 4 years for 293 patients who could be followed over 4 years.

\begin{tabular}{lll}
\hline Year & No. who continued & RR \\
\hline 1 & 218 & $74.4 \%$ \\
2 & 203 & $69.3 \%$ \\
3 & 185 & $63.1 \%$ \\
4 & 163 & $55.6 \%$ \\
\hline
\end{tabular}

Table 3 Reasons for discontinuing LTG during the 4-year follow-up ( $\mathrm{n}=293)$.

Reasons for discontinuing LTG

\begin{tabular}{lrl}
\hline Lack of efficacy & 56 & $(19.1 \%)$ \\
Adverse events & 36 & $(12.3 \%)$ \\
Both & 22 & $(7.5 \%)$ \\
Other & 9 & $(3.1 \%)$ \\
Unknown & 7 & $(2.4 \%)$ \\
\hline
\end{tabular}


Table 4 Seizure reduction in patients who continued LTG $(n=293)$.

\begin{tabular}{lllllllll}
\hline & \multicolumn{7}{l}{ change from } \\
\cline { 2 - 8 } & $0-6$ & $6-12$ & $12-18$ & $18-24$ & $24-30$ & $30-36$ & $36-42$ & $42-48$ \\
& months & months & months & months & months & months & months & months \\
\hline Seizure-free for & 30 & 25 & 21 & 27 & 28 & 26 & 38 & 35 \\
6 months & $10.3 \%$ & $10.4 \%$ & $9.6 \%$ & $13.0 \%$ & $13.8 \%$ & $13.3 \%$ & $20.9 \%$ & $20.8 \%$ \\
Lower seizure & 78 & 67 & 49 & 39 & 30 & 38 & 29 & 28 \\
frequency & $26.6 \%$ & $27.8 \%$ & $22.5 \%$ & $18.8 \%$ & $14.8 \%$ & $19.4 \%$ & $15.9 \%$ & $16.7 \%$ \\
No efficacy & 90 & 78 & 81 & 66 & 69 & 56 & 58 & 57 \\
& $30.7 \%$ & $32.4 \%$ & $37.2 \%$ & $31.9 \%$ & $34.0 \%$ & $28.6 \%$ & $31.9 \%$ & $33.9 \%$ \\
Higher seizure & 51 & 35 & 31 & 38 & 29 & 31 & 18 & 13 \\
frequency & $17.4 \%$ & $14.5 \%$ & $14.2 \%$ & $18.4 \%$ & $14.3 \%$ & $15.8 \%$ & $9.9 \%$ & $7.7 \%$ \\
Not reported & 44 & 36 & 36 & 37 & 47 & 45 & 39 & 35 \\
& $15.0 \%$ & $14.9 \%$ & $16.5 \%$ & $17.9 \%$ & $23.2 \%$ & $23.0 \%$ & $21.4 \%$ & $20.8 \%$ \\
Total & 293 & 241 & 218 & 207 & 203 & 196 & 182 & 168 \\
& $100 \%$ & $100 \%$ & $100 \%$ & $100 \%$ & $100 \%$ & $100 \%$ & $100 \%$ & $100 \%$ \\
\hline
\end{tabular}

Table 5 Seizure reduction in patients who discontinued LTG during the follow-up period.

\begin{tabular}{llcrrrr}
\hline Months & Seizure free & $\begin{array}{l}\text { Lower seizure } \\
\text { frequency }\end{array}$ & No effect & $\begin{array}{l}\text { Higher seizure } \\
\text { frequency }\end{array}$ & Not reported & Total \\
\hline 6 & $2(3.8 \%)$ & $1(1.9 \%)$ & $13(25.0 \%)$ & $22(42.3 \%)$ & $14(26.9 \%)$ & $52(100 \%)$ \\
12 & $2(8.7 \%)$ & $2(8.7 \%)$ & $8(34.8 \%)$ & $8(34.8 \%)$ & $3(13.0 \%)$ & $23(100 \%)$ \\
18 & $0(0 \%)$ & $1(16.7 \%)$ & $6(60 \%)$ & $2(20 \%)$ & $1(10 \%)$ & $10(100 \%)$ \\
24 & $0(0 \%)$ & $1(16.7 \%)$ & $2(33.3 \%)$ & $2(33.3 \%)$ & $1(16.7 \%)$ & $6(100 \%)$ \\
30 & $0(0 \%)$ & $0(0 \%)$ & $3(60 \%)$ & $1(20 \%)$ & $1(20 \%)$ & $5(100 \%)$ \\
36 & $0(0 \%)$ & $1(6.7 \%)$ & $8(53.3 \%)$ & $3(20.0 \%)$ & $3(20 \%)$ & $15(100 \%)$ \\
42 & $1(11.1 \%)$ & $1(11.1 \%)$ & $6(66.7 \%)$ & $1(11.1 \%)$ & $0(0 \%)$ & $9(100 \%)$ \\
48 & $1(10 \%)$ & $1(10 \%)$ & $6(60 \%)$ & $2(20 \%)$ & $0(0 \%)$ & $10(100 \%)$ \\
Total & $6(4.6 \%)$ & $8(6.2 \%)$ & $52(40.0 \%)$ & $41(31.5 \%)$ & $23(17.7 \%)$ & $130(100 \%)$ \\
\hline
\end{tabular}

Table 6 Number of patients in seizure remission $(n=293)$.

\begin{tabular}{lrr}
\hline Months & $\mathrm{N}$ & $\%$ \\
\hline 6 & 30 & 10.2 \\
12 & 12 & 4.1 \\
24 & 5 & 1.7 \\
36 & 5 & 1.7 \\
48 & 4 & 1.4 \\
\hline
\end{tabular}

Table 7 Most frequently reported side effects in the total study population $(n=314)$.

\begin{tabular}{rlll}
\hline 6 months & Dizziness $14.6 \%$ & More active $12.6 \%$ & More active/more responsive $9.1 \%$ \\
12 months & Dizziness $14.3 \%$ & More active $12.0 \%$ & Headache $9.8 \%$ \\
18 months & Dizziness $22.6 \%$ & More active $15.1 \%$ & Tiredness $10.8 \%$ \\
24 months & Dizziness $13.8 \%$ & More active $12.6 \%$ & Tiredness $9.2 \%$ \\
\hline
\end{tabular}


positive effect of the drug. A large percentage of patients experienced this effect. They felt more active and more responsive.

The most frequently reported side effect at discontinuation (Table 8 ) was dizziness (15.7\%). Headache was the second most frequently reported side effect (8.4\%). Being/ feeling "more active" was reported by $7.7 \%$ of the patients.

The plasma LTG level was measured 277 times in the patient group. At each 6-month interval, comedications taken by the patients were recorded. Every patient was classified into one of the following groups at each interval: taking LTG and a CYP3A4 inducer (phenobarbital, primidone, phenytoin, carbamazepine), taking LTG and valproate, taking LTG and both an inducer and valproate, or taking LTG in monotherapy. By monotherapy is meant only LTG or LTG together with medication that has no LTG enzyme-inducing or -inhibiting action. Figure 2 illustrates the plasma levels measured for the different doses of LTG relative to the comedication. Seven plasma levels were above the recommended upper limit of $14 \mathrm{mg} / \mathrm{L}$ (five in those on LTG monotherapy, one in a patient taking LTG with valproate and an inducer, and one in a patient taking LTG with an inducer). Seventy-one plasma levels were below the recommended lower limit of $3 \mathrm{mg} / \mathrm{L}$ ( 9 in those taking LTG with an inducer and valproate, 1 in a patient on monotherapy, and 61 times in those taking LTG with an inducer).

Figure 2 also demonstrates that patients taking LTG in combination with valproate never received more than $400 \mathrm{mg}$ LTG daily. However, when taking LTG in combination with an inducer, some patients received $1200 \mathrm{mg}$ LTG daily. Many dosages are above the recommendations of the Dutch SMPC of GlaxoSmithKline. In Version 21.1, October 2006, they recommended a lamotrigine dosage between 100 and $500 \mathrm{mg}$. In combination with an inducer, they recommended a LTG dosage between 200 and 400 mg daily, and in combination with valproate, a dosage between 100 and $200 \mathrm{mg}$ daily. These recommendations are illustrated in Figure 2 as vertical lines. Higher-thanrecommended dosages were prescribed for $42 \%$ of the patients on monotherapy; for $62 \%$ of the patients taking LTG with an inducer; and $63 \%$ of the patients taking LTG and valproate.

That a combination of valproate and LTG results in higher plasma LTG levels is confirmed by Figure 2. The slope of the trend line is inversely related to LTG clearance.

Clearance of LTG taken in combination with valproate is 56\% that of LTG monotherapy. Clearance of LTG taken in combination with an enzyme inducer is $190 \%$ that of LTG monotherapy. These values correlate well with the dosage recommendations of GlaxoSmithKline. The manufacturer recommends half the dosage of LTG monotherapy when the patient also takes valproate. The patient taking LTG with an enzyme inducer requires two times the dose used in monotherapy.

Mean plasma levels were calculated for the four groups. Patients taking LTG in monotherapy had a mean plasma level of $8.7 \mathrm{mg} / \mathrm{L}$ (SD 4.0). Patients taking valproate 
Table 8 Adverse events reported by patients who discontinued treatment.

\begin{tabular}{lll}
\hline Adverse event & Frequency & $\%$ \\
\hline Dizziness & 41 & 15.7 \\
Headache & 22 & 8.4 \\
More active & 20 & 7.7 \\
Skin reaction & 19 & 7.3 \\
Sleeping complaints/ insomnia & 19 & 7.3 \\
Sleeping complaints/ sleepiness & 17 & 6.5 \\
Vision disorders & 16 & 6.1 \\
Exhaustion & 16 & 6.1 \\
More clear/ more responsive & 12 & 4.6 \\
Gastrointestinal complaints & 10 & 3.8 \\
Mood disorders - agitation & 9 & 3.4 \\
Other non-CZS & 8 & 3.1 \\
Tremor & 7 & 2.7 \\
Mood disorders - aggression & 7 & 2.7 \\
Endocrine complaints & 7 & 2.7 \\
Other CZS & 6 & 2.3 \\
Cardiovascular & 6 & 2.3 \\
Ataxia & 5 & 1.9 \\
Mood disorders - hyperirritability & 4 & 1.5 \\
Rheumatologic & 3 & 1.1 \\
Confusion & 2 & 0.8 \\
Mood disorders - depression & 1 & 0.4 \\
Mood disorders - apathy & 1 & 0.4 \\
Eye complaints & 1 & 0.4 \\
Otorhinolaryngology (ENT) & 1 & 0.4 \\
Asthenia & & 0.4 \\
\hline
\end{tabular}

had a mean plasma level of $8.7 \mathrm{mg} / \mathrm{L}$ (SD 2.4). According to a two-tailed independentsample $t$ test with a significance level of 0.05 , these groups did not differ with respect to plasma level $(\mathrm{P}=0.960)$. The plasma levels of patients taking an inducer $(4.8 \mathrm{mg} / \mathrm{L}$, SD 2.6) or an inducer and valproate $(4.9 \mathrm{mg} / \mathrm{L}, \mathrm{SD} 1.9)$ in addition to $\mathrm{LTG}$ were much lower. The latter two groups did not differ from each other $(\mathrm{P}=0.797)$, but did differ from the first two groups $(\mathrm{P}<0.001)$.

In this population the mean dosage of LTG in patients on monotherapy was 529 mg (SD 208). Patients taking LTG in combination with an inducer received a mean dosage of $533 \mathrm{mg}$ (SD 222). These two mean dosages are the same $(\mathrm{P}=0.914)$, which exemplifies the low plasma levels of patients taking LTG and an inducer.

The numbers of side effects of LTG therapy observed in groups with different plasma levels (Table 9) indicates that the number of negative side effects per total number of patients increased with plasma level. We did not find a relationship between specific side effects and plasma levels. 


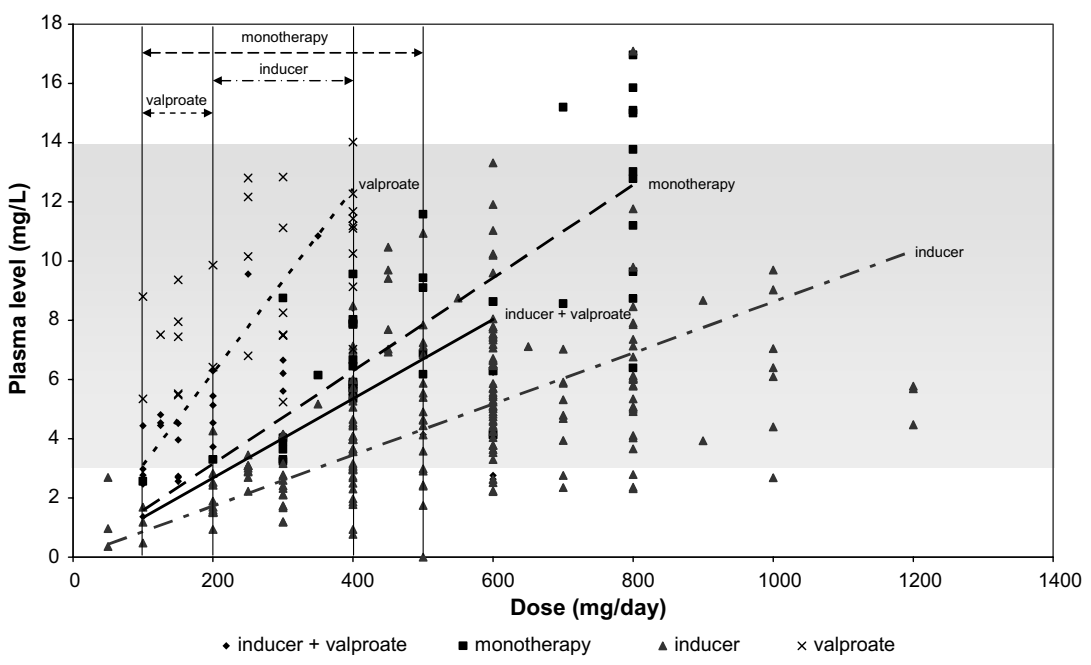

Figure 2 Plasma LTG level versus dose.

The relationship between plasma LTG levels and gender was tested with an independent sample t test. The mean plasma LTG level in males was $5.6 \mathrm{mg} / \mathrm{L}$ (SD 3.4), and in females, $5.5 \mathrm{mg} / \mathrm{L}$ (SD 2.8). There was no significant difference in mean plasma LTG levels between the genders.

Of the total 265 plasma level measurements, 60 were in patients ( $>18$ years old) who discontinued LTG therapy.

Most patients $(\mathrm{N}=39)$ discontinued LTG therapy because of lack of efficacy (mean plasma level $=5.6 \mathrm{mg} / \mathrm{L}, \mathrm{SD} 2.6$ ).

Patients who discontinued therapy because of adverse events, because of both lack of efficacy and adverse events, or for an unknown reason had respective mean plasma levels of 5.2 (SD 2.4), 4.9 (SD 2.3), and 3.5 (SD 2.5) mg/L.

These data show that there is no relationship between reason for discontinuation and plasma LTG level. We examined the plasma LTG levels of patients who continued LTG therapy and patients who discontinued LTG therapy.

There was no difference between these groups with respect to mean plasma LTG levels. Patients who continued therapy had a mean plasma level of 5.7 (SD 3.3) mg/L, and patients who discontinued therapy had a mean plasma level of 5.2 (SD 2.5) $\mathrm{mg} / \mathrm{L}$.

The mean LTG doses of groups with different reasons for discontinuation are listed in Table 10. The dosage for those who cited lack of efficacy as their reason for discontinuation was significantly higher than the dosages of those who cited adverse events $(\mathrm{P}=0.016)$ and those who cited both $(\mathrm{P}=0.016)$. 
Table 9 Negative side effects versus plasma level.

\begin{tabular}{lll}
\hline Plasma level $(\mathrm{mg} / \mathrm{L})$ & \multicolumn{2}{l}{ No. of side effects/total no. of patients } \\
\hline $0-4$ & $44 / 95$ & $(46.3 \%)$ \\
$4-8$ & $85 / 134$ & $(63.4 \%)$ \\
$8-12$ & $32 / 36$ & $(88.9 \%)$ \\
$12-16$ & $10 / 11$ & $(90.9 \%)$ \\
\hline
\end{tabular}

Table 10 Reason for discontinuation versus dose and comedication.

\begin{tabular}{llcccc}
\hline Reason for discontinuation & $\begin{array}{l}\text { Mean LTG } \\
\text { dose }(\mathrm{mg})\end{array}$ & $\mathrm{N}$ & $\begin{array}{l}\text { SD } \\
(\mathrm{mg})\end{array}$ & $\begin{array}{l}\text { Minimum } \\
(\mathrm{mg})\end{array}$ & $\begin{array}{l}\text { Maximum } \\
(\mathrm{mg})\end{array}$ \\
\hline Lack of efficacy & 609 & 39 & 257 & 50 & 1200 \\
Adverse events & 363 & 8 & 234 & 50 & 600 \\
Both & 336 & 7 & 195 & 50 & 600 \\
Other & - & 0 & - & - & - \\
Unknown & 400 & 6 & 253 & 100 & 700 \\
Total & 523 & 60 & 269 & 50 & 1200 \\
\hline
\end{tabular}

To determine which variables predicted continuation and discontinuation of LTG therapy, the differences between patients who continued and those who discontinued LTG after 12 months were evaluated. Dose was significantly lower in patients who discontinued LTG therapy than in patients who continued therapy. But the total drug load $\left(\sum(\mathrm{AED} \text { dose/DDD })\right)^{23}$ without LTG was higher in patients who discontinued within 12 months. In terms of percentage, patients who discontinued therapy seemed to have experienced more negative side effects and more positive side effects. In addition to these differences between patients, there were no variables that predicted either continuation or discontinuation.

\section{I S C U S S O N}

The RR of a drug can be used as a predictor of the efficacy and tolerability of that drug. The long-term retention of LTG has been described in several other studies. Collins et al. ${ }^{24}$ compared gabapentin (GBP), LTG, topiramate (TPM), and vigabatrin (VGB). It appeared that LTG was the drug least likely to be discontinued. The time to $50 \%$ dropout, expressed in months, was $>43$ months for LTG in contrast to 13 months for GBP, 9.5 months for TPM, and 29 months for VGB. In the study by Lhatoo et al. ${ }^{25}$, more patients appeared to continue TPM compared with LTG or GBP. At the end of the first year of therapy, RRs were 52\% (TPM), 46\% (LTG), and 23\% (GBP). The difference between the RRs for TPM and LTG was not statistically significant. The 
main reason for discontinuing LTG therapy was lack of efficacy; the second reason was adverse effects. Wong et al. ${ }^{26}$ described the long-term use of GBP, LTG, and VGB in patients with chronic epilepsy. Patients were less likely to discontinue LTG than GBP or VGB. Kaplan-Meier analysis of all 1050 patients yielded estimated RRs for LTG of $60 \%$ at 1 year, $40 \%$ at 5 years, and $38 \%$ at 8 years after the start of therapy ${ }^{27}$.

Faught et al. ${ }^{28}$ performed a 6 -year continuation study with LTG. They concluded that long-term LTG use was associated with a low incidence of adverse effects. The added value of our study lies in the large number of patients included $(n=314)$, the long period of evaluation, and the unselected group (all patients who received LTG treatment in our center). The RRs of LTG in this study after 1, 2, 3, and 4 years were 74.4, 69.3, 63.1, and 55.6\%, respectively. This contrasts with the RR for topiramate of $38.3 \%$ after 2 years and that for levetiracetam of $45.8 \%$ after 2 years ${ }^{2,3}$. Most patients discontinued LTG therapy because of lack of efficacy. However, the efficacy was not greater in patients who continued LTG therapy. It is possible that patients with unsatisfactory seizure control continued LTG nonetheless because of its positive side effects. In general, LTG was very well tolerated. A special observation is that rash did not occur in the included patients.

Plasma LTG levels are described in many publications. In older publications, the recommended plasma level was $1-3 \mathrm{mg} / \mathrm{L}^{10-16}$. Since 1998, a plasma LTG level of 3-14 $\mathrm{mg} / \mathrm{L}$ has been recommended ${ }^{18-22}$. There is high variability in the plasma levels measured in the total patient group in this study. This may be caused by interindividual variability or the time between intake of LTG and measurement. Because of the large number of measurements, this variability does not influence trend lines. Of 277 plasma LTG levels measured, 199 were between 3 and $14 \mathrm{mg} / \mathrm{L}, 7$ were above $14 \mathrm{mg} / \mathrm{L}$, and 71 were below $3 \mathrm{mg} / \mathrm{L}$. To achieve plasma levels as high as 14 $\mathrm{mg} / \mathrm{L}$, a LTG dosage higher than that recommended by the manufacturer was used. Among patients on monotherapy, higher-than-recommended dosages were used in 42\%; among patients taking LTG with an inducer, $62 \%$; and in patients taking LTG with valproate, $63 \%$. The manufacturer recommends that patients taking LTG and valproate receive half the dosage of LTG monotherapy, and that patients taking LTG with an inducer receive twice the monotherapy dose. These recommendations were confirmed by this study. The mean plasma LTG level for patients taking LTG with an inducer $(4.8 \mathrm{mg} / \mathrm{L})$ was lower than that of patients on LTG monotherapy or on LTG and valproate ( 8.7 and $8.7 \mathrm{mg} / \mathrm{L}$, respectively).

After this study, it is still difficult to provide variables that predict continuation or discontinuation. Only the drug load can predict continuation or discontinuation. The drug load without LTG was higher in patients who discontinued within 12 months. The LTG dose was significantly lower in patients who discontinued within 12 months. The plasma LTG level could not be used to predict discontinuation or continuation. The mean plasma level was the same for both groups. 


\section{R E FER E NCES}

1. Shorvon SD, Fish DR, Perucca E, Dodson WE. The treatment of epilepsy. 2nd ed. London: Blackwell Science; 2004.

2. Bootsma HP, Coolen F, Aldenkamp AP, et al. Topiramate in clinical practice: long-term experience in patients with refractory epilepsy referred to a tertiary epilepsy center. Epilepsy Behav 2004;5:380-7.

3. Bootsma HP, Ricker L, Diepman L, et al. Levetiracetam in clinical practice: long-term experience in patients with refractory epilepsy referred to a tertiary epilepsy center. Epilepsy Behav 2007;10:296303.

4. Sander JW. New antiepileptic drugs in practice: how do they perform in the real world? Acta Neurol Scand 2005;112:26-9.

5. Mohanraj R, Brodie MJ. Measuring the efficacy of antiepileptic drugs. Seizure 2003;12:413-43.

6. Zacarra G, Messori A, Cincotta M, et al. Comparison of the efficacy and tolerability of new antiepileptic drugs: what can we learn from long-term studies? Acta Neurol Scand 2006;114:157-68.

7. Chadwick D. Better comparisons of antiepileptic drugs: what measures of efficacy? Pharm World Sci 1997;19:214-6.

8. Bartoli A, Guerrini R, Belmonte A, Alessandri MG, Gatti G, Perucca E. The influence of dosage, age, and comedication on steady state plasma lamotrigine concentrations in epileptic children: a prospective study with preliminary assessment of correlations with clinical response. Ther Drug Monit 1997;19:252-60.

9. Luckasson R, Coulter DL, Polloway EA. Mental retardation: definition, classification, and systems of supports. Washington, DC: Am. Assoc. on Ment. Retard; 1992.

10. Binnie CD, Debets RM, Engelsman M, et al. Double-blind crossover trial of lamotrigine (Lamictal) as add-on therapy in intractable epilepsy. Epilepsy Res 1989;4:222-9.

11. Brodie MJ. Lamotrigine. Lancet 1992;339:1397-400.

12. Jawad S, Richens A, Goodwin G, Yuen WC. Controlled trial of lamotrigine (Lamictal) for refractory partial seizures. Epilepsia 1989;30:356-63.

13. Loiseau P, Yuen AW, Duche B, Menager T, Arne-Bes MC. A randomised double-blind placebocontrolled crossover add-on trial of lamotrigine in patients with treatment-resistant partial seizures. Epilepsy Res 1990;7:136-45.

14. Matsuo F, Bergen D, Faught E, et al. for the U.S. Lamotrigine Protocol 05 Clinical Trial Group. Placebo-controlled study of the efficacy and safety of lamotrigine in patients with partial seizures. Neurology 1993;43:2284-91.

15. Messenheimer J, Ramsay RE, Willmore LJ, et al. Lamotrigine therapy for partial seizures: a multicenter, placebo-controlled, double-blind, cross-over trial. Epilepsia 1994;35:113-21.

16. Schapel GJ, Beran RG, Vajda FJ, et al. Double-blind, placebo controlled, crossover study of lamotrigine in treatment resistant partial seizures. J Neurol Neurosurg Psychiatry 1993;56:448-53.

17. Hirsch LJ, Weintraub D, Du Y, et al. Correlating lamotrigine serum concentrations with tolerability in patients with epilepsy. Neurology 2004;63:1022-6. 
18. Morris RG, Lee MY, Cleanthous X, Black AB. Long-term follow-up using a higher target range for lamotrigine monitoring. Ther Drug Monit 2004;26:626-32.

19. Morris RG, Black AB, Harris AL, Batty AB, Sallustio BC. Lamotrigine and therapeutic drug monitoring: retrospective survey following the introduction of a routine service. Br J Clin Pharmacol 1998;46:547-51.

20. Neels HM, Sierens AC, Naelaerts K, Scharpe SL, Hatfield GM, Lambert WE. Therapeutic drug monitoring of old and newer antiepileptic drugs. Clin Chem Lab Med 2004;42:1228-55.

21. Perucca E. Is there a role for therapeutic drug monitoring of new anticonvulsants? Clin Pharmacokinet 2000;38:191-204.

22. Froscher W, Keller F, Vogt H, Kramer G. Prospective study on concentration-efficacy and concentration-toxicity: correlations with lamotrigine serum levels. Epileptic Disord 2002;4:49-56.

23. Deckers CL, Hekster YA, Keyser A, Meinardi H, Renier WO. Drug load in clinical trials: a neglected factor. Clin Pharmacol Ther 1997;62:592-5.

24. Collins TL, Petroff OA, Mattson RH. A comparison of four new antiepileptic medications. Seizure 2000;9:291-3.

25. Lhatoo SD, Wong IC, Polizzi G, Sander JW. Long-term retention rates of lamotrigine, gabapentin, and topiramate in chronic epilepsy. Epilepsia 2000;41:1592-6.

26. Wong IC, Chadwick DW, Fenwick PB, Mawer GE, Sander JW. The long-term use of gabapentin, lamotrigine, and vigabatrin in patients with chronic epilepsy. Epilepsia 1999;40:1439-45.

27. Wong IC, Mawer GE, Sander JW, Lhatoo SD. A pharmacoepidemiologic study of factors influencing the outcome of treatment with lamotrigine in chronic epilepsy. Epilepsia 2001;42:1354-8.

28. Faught E, Matsuo FE, Schachter S, Messenheimer J, Woble GP. Long-term tolerability of lamotrigine; data from a 6-year continuation study. Epilepsy Behavior 2004;5:31-6. 


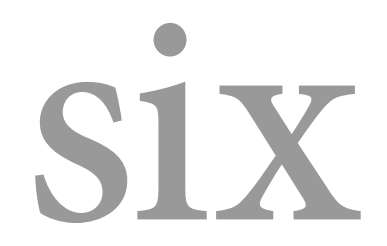

\section{Long-term effects of levetiracetam and topiramate in clinical practice: A head-to-head comparison}

H.P.R. Bootsma, L. Ricker, L. Diepman, J. Gehring, J. Hulsman, D. Lambrechts, L. Leenen, M. Majoie, A. Schellekens, M. de Krom, A.P. Aldenkamp Seizure (2008) 17, 19-26 
Objective: Two of the most commonly prescribed new antiepileptic drugs as add-on therapy for patients with chronic refractory epilepsies are topiramate and levetiracetam. In regulatory trials, both drugs were characterized as very promising new antiepileptic drugs. However, results from these highly controlled short-term clinical trials cannot simply be extrapolated to everyday clinical practice, also because headto-head comparisons are lacking. Therefore, results from long-term open label observational studies that compare two or more new AEDs are crucial to determine the long-term performance of competing new antiepileptic drugs in clinical practice.

Method: We analyzed all patients referred to a tertiary epilepsy centre who had been treated with topiramate from the introduction of the drug in spring 1993 up to a final assessment point mid-2002 and all patients who had been treated with LEV in the same centre from the introduction of the drug in early 2001 up to a final assessment point end-2003 using a medical information system.

Results: Three hundred and one patients were included for levetiracetam and 429 patients for TPM. Retention rate after 1 year was $65.6 \%$ for LEV-treated patients and $51.7 \%$ for TPM-treated patients $(\mathrm{p}=0.0015)$. Similarly, retention rates for LEV were higher at the 24-month mark: $45.8 \%$ of LEV-treated patients and $38.3 \%$ of TPMtreated patients were still continuing treatment $(\mathrm{p}=0.0046)$. Adverse events led to drug discontinuation in $21.9 \%$ of TPM-treated patients compared to $6.0 \%$ of LEVtreated patients $(\mathrm{p}<0.001)$. The number of patients discontinuing treatment because of lack of efficacy was similar for both groups. Seizure freedom rates varied between 11.6 and $20.0 \%$ for TPM and between 11.1 and 14.3\% for LEV per 6-months interval. Several important AED specific adverse events leading to drug discontinuation were identified, including neurocognitive side effects from TPM and mood disorders from LEV.

Conclusion: The retention rate for LEV is significantly higher than for TPM. LEV had a more favourable side effect profile than TPM with comparable efficacy. Patients on TPM discontinued treatment mainly because of neurocognitive side effects. In the treatment with LEV, the effects on mood must not be underestimated. 
The introduction of several new antiepileptic drugs (AEDs) in the past decades may be a welcome development for patients with refractory epilepsy. ${ }^{1}$ Two of the most commonly prescribed "new generation" AEDs in the Netherlands are topiramate (TPM) and levetiracetam (LEV). Both drugs were found to be an effective and safe therapeutic option as adjunctive therapy for partial seizures in adults during the regulatory well-controlled trials. ${ }^{2-9}$ Even though only TPM has also FDA approval for primary generalized seizures, evidence is growing that LEV may be a useful broad-spectrum AED as well..$^{10}$ The most frequently reported side effects associated with the use of LEV in pooled analyses of the regulatory trials were somnolence, asthenia, headache and dizziness. ${ }^{11}$ TPM-associated adverse events were predominantly central nervous system-related symptoms, including somnolence, dizziness and psychomotor slowing. ${ }^{12}$

However, these clinical trials were designed for registration purposes. Regulatory studies only show antiepileptic activity compared with placebo. It is impossible to transfer findings derived from such trials to everyday clinical practice ${ }^{13}$ and make comparisons between AEDs. In order to overcome the shortcomings of those trials and to provide data that can help clinicians in making treatment decisions, other methods must be used to compare competing new AEDs. Ideally, evidence-based treatment guidelines will use information from large multicenter randomized controlled trials that compare AEDs. These studies are, however, rarely performed. Therefore, results from meta-analysis and long-term open label observational studies may give clinicians a possibility to compare the newer AEDs despite the limitations of indirect comparisons.

One way to compare the long-term performance of AEDs in clinical practice is to evaluate retention rates. Retention rate is considered to be a composite of drug efficacy and drug safety and expresses the willingness of patients to continue drug treatment. It is therefore clinically the most relevant parameter of an antiepileptic drug. ${ }^{14}$ Although TPM and LEV were characterized as very promising AEDs in regulatory trials, a long-term study that retrospectively compared the retention rates of five new AEDs has shown retention rates of $44 \%$ for TPM and $54 \%$ for $\mathrm{LEV}^{15}$ after 2 years of follow-up. This implies that about half of the patients will discontinue treatment within a period of 2 years. Prospective data from long-term open-label studies showed seizure freedom rates of about $10 \%$ for at least 6 months in patients on TPM and LEV. However, withdrawal rates due to adverse events were higher for TPM. ${ }^{16,17}$

Presently, only few data are available to show how TPM compares to LEV in the long-term in a single tertiary epilepsy centre. We therefore performed a systematic audit of TPM and LEV in the 'real life' setting of our centre with the aim to compare 
the long-term effects of both drugs in a broad population, specifically retention time, efficacy and tolerability in clinical practice.

\section{METHOD}

The study was approved by the Medical Ethics Committee. All patients who had been treated with TPM in the Epilepsy Centre Kempenhaeghe from the introduction of the drug in spring 1993 up to a final assessment point mid-2002 and all patients who had been treated with LEV in the same centre from the introduction of the drug in early 2001 up to a final assessment point end-2003 were identified by means of our automated medical information system (MIS) and subsequently analyzed. Patients whom had TPM or LEV prescribed initially elsewhere were not included. The followup period for both drugs was 24 months. A standardized data form was developed. The data were obtained from our MIS and individual patient medical records. Variables that were included in the database were patient characteristics (age, sex, weight, age at onset of seizures, duration of epilepsy at the introduction of drug treatment, mental retardation [defined as IQ < 70], CT/MRI abnormalities), length of treatment, efficacy, reported side effects and reasons for discontinuation. Epilepsy and seizures were classified using the International League Against Epilepsy (ILAE) classification. Treatment was evaluated every 6 months for both drugs. Efficacy was measured using a 5-point scale: seizure remission, any reduction in seizure frequency, no efficacy, any increase in seizure frequency or not classified. Reason for discontinuation of LEV and TPM was entered into the database using a 4-point scale: no efficacy, adverse events, both or other. Mood disorders due to use of AEDs were classified into activating and sedating effects, with the former leading to aggression, hyperirritability and agitation, and the latter leading to apathy and depression. ${ }^{18,19}$ Data forms were entered into a computerized database for analysis. Statistical analysis was performed using SPSS 15.0 for Windows. Retention rates were calculated by using Kaplan-Meier survival analysis, ${ }^{20}$ and comparisons between the retention curves of TPM and LEV were analyzed using log-rank tests. Comparison between both drugs in terms of seizure remission and reason for discontinuation was analyzed using Pearson $\chi^{2}$.

\section{RES U L T S}

Three hundred and four patients were identified using LEV and 470 patients using TPM. For LEV, three patients were excluded; one patient was already exposed to LEV on referral to our centre and of two patients data were lacking. For TPM, 41 patients were excluded; 37 patients were already exposed to TPM on referral to our centre and of 4 patients data were lacking. 
Table 1 Patient demographics and characteristics.

\begin{tabular}{|c|c|c|}
\hline & Levetiracetam & Topiramate \\
\hline Total no. of patients & 301 & 429 \\
\hline Age & $32.5 \pm 16.8$ & $34.9 \pm 8.6$ \\
\hline Age range & $1-75$ & $1-73$ \\
\hline Age distribution & $19.9 \%<18$ & $24.5 \%<18$ \\
\hline $\operatorname{Sex}(F / M)$ & $156 / 145$ & $204 / 225$ \\
\hline Weight (kg) & $50.5 \pm 21.6$ & $54.0 \pm 26.6$ \\
\hline \multicolumn{3}{|l|}{ Type of epilepsy } \\
\hline Localization-related & $217(72.1 \%)$ & $323(75.3 \%)$ \\
\hline Generalized (including secondarily generalized) & $54(17.9 \%)$ & $86(20.1 \%)$ \\
\hline Undetermined & $16(5.4 \%)$ & $10(2.3 \%)$ \\
\hline Not classified & $14(4.6 \%)$ & $10(2.3 \%)$ \\
\hline \multicolumn{3}{|l|}{ Seizure type } \\
\hline Partial onset & $243(80.7 \%)$ & $352(82.1 \%)$ \\
\hline Generalized & $138(45.8 \%)$ & $209(48.7 \%)$ \\
\hline Not classified & $19(6.3 \%)$ & $19(4.4 \%)$ \\
\hline Nonepileptic attacks & $28(9.3 \%)$ & $31(7.2 \%)$ \\
\hline Age at onset of seizures & $11.7 \pm 11.7$ & $9.7 \pm 11.1$ \\
\hline Duration of epilepsy before drug introduction (years) & $21.3 \pm 12.9$ & $21.1 \pm 13.4$ \\
\hline No. of mentally retarded & $98(32.6 \%)$ & $158(36.8 \%)$ \\
\hline CT abnormalities & $17.3 \%$ & $18.9 \%$ \\
\hline MRI abnormalities & $31.9 \%$ & $36.4 \%$ \\
\hline No. of concomitant AEDs & $2.16 \pm 0.827$ & $2.04 \pm 0.779$ \\
\hline \multicolumn{3}{|l|}{ Most frequently used concomitant AEDs at baseline } \\
\hline carbamazepine & $39.9 \%$ & $44.8 \%$ \\
\hline clobazam & $37.6 \%$ & $34.3 \%$ \\
\hline phenytoin & $15.6 \%$ & $19.3 \%$ \\
\hline lamotrigine & $42.5 \%$ & $47.1 \%$ \\
\hline oxcarbazepine & $16.3 \%$ & $14.5 \%$ \\
\hline valproic acid & $19.3 \%$ & $18.4 \%$ \\
\hline
\end{tabular}

Table 1 lists the main characteristics of the patients. The mean age was $32.5 \pm 16.8$ years for LEV and 34.9 \pm 8.6 years for TPM. There was an equal distribution with respect to gender. Age at onset of seizures was in the 11th and 9th year, respectively, with a duration of active epilepsy (= years with seizures) of more than 20 years. Localizationrelated epilepsy was by far the most prevalent type of epilepsy and, consequently, partial onset seizures the most frequently recorded type of seizure. Children and 


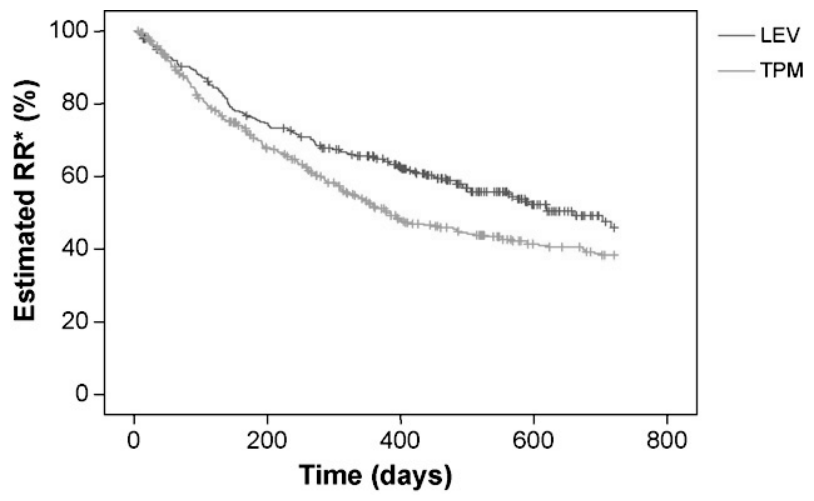

Figure 1 Comparison between the Kaplan-Meier survival curves of TPM and LEV ( ${ }^{*} \mathrm{RR}$ : retention rate).

mentally handicapped patients were substantially and equally represented in both study groups. The number of patients with abnormalities on CT and MRI were similar for the two groups. No difference could be identified in the number of concomitant AEDs and the most frequently used AEDs. Thus, both populations were comparable for the most important demographic and clinical variables and represent the typical group of refractory patients referred to a tertiary epilepsy centre.

Retention rates for TPM and LEV are illustrated with Kaplan-Meier survival curves in Figure 1. Retention rate after 1 year was $65.6 \%$ for LEV-treated patients and $51.7 \%$ for TPM-treated patients $(\mathrm{p}=0.0015)$. Similarly, retention rates for LEV were significantly higher at the 24 -month mark: $45.8 \%$ of LEV-treated patients and $38.3 \%$ of TPM-treated patients were still continuing treatment $(\mathrm{p}=0.0046)$. However, at 6 months follow-up, there was no significant difference between the two profiles ( $\mathrm{p}=$ 0.1088). For both drugs, it is shown that the largest percentage of discontinuations occurred in the first period of treatment with a rapid drop of retention rates until about 400 days, after which a plateau seems to be reached.

The main reasons for drug discontinuation are shown in Figure 2. Adverse events led to drug discontinuation in $21.9 \%$ of TPM-treated patients compared to $6.0 \%$ of LEV-treated patients $(\mathrm{p}<0.001)$. The number of patients discontinuing treatment because of lack of efficacy or the combination of lack of efficacy and adverse events was similar for both groups.

For LEV, adverse events were the most important reason for drug discontinuation during the first 3 months. Thereafter, lack of efficacy played the most important role in drug discontinuation. For TPM, however, tolerability issues lasted much longer and were the main reason for drug discontinuation in the first 18 months. 


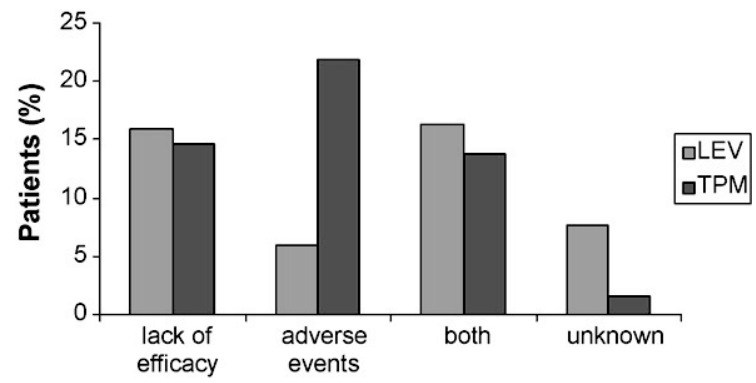

Figure 2 Reason for discontinuation; comparison between TPM and LEV.

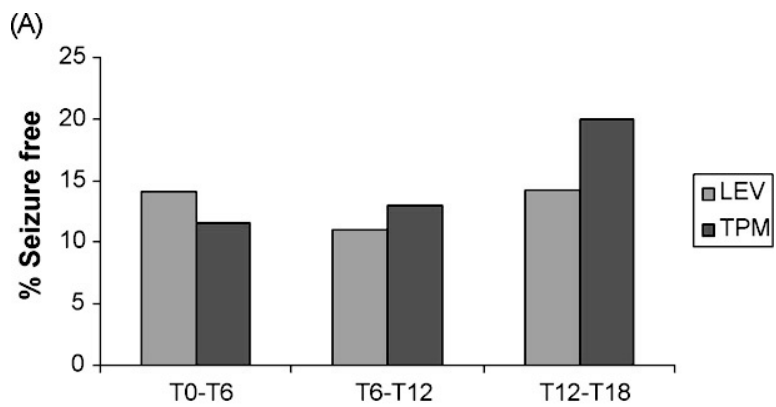

Figure 3A Seizure freedom rates per 6-month interval; comparison between TPM and LEV.

(B)

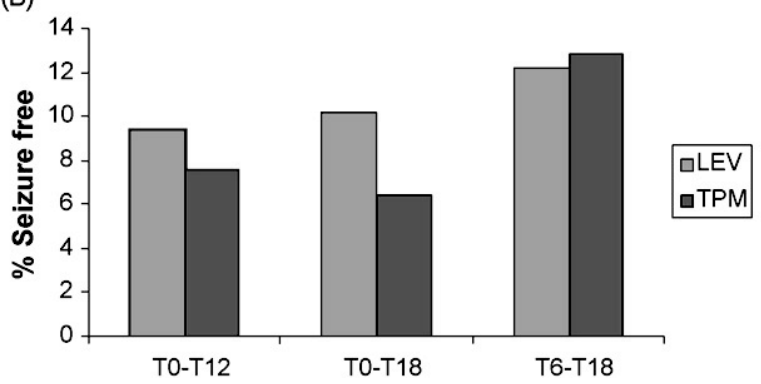

Figure 3B Seizure remission for at least one year; comparison between TPM and LEV. 
Seizure freedom rates per 6-month interval are illustrated in Figure 3A. Seizure freedom rates varied between 11.6 and $20.0 \%$ for TPM and between 11.1 and $14.3 \%$ for LEV. No statistically significant differences between TPM and LEV could be identified at 6 months $(\mathrm{p}=0.468), 12$ months $(\mathrm{p}=0.653)$ and 18 months $(\mathrm{p}=0.244)$. Figure $3 \mathrm{~B}$ shows the number of patients completely seizure-free for at least 1 year. Seizure remission rates for the intervals T0-T12 and T0-T18 were higher for LEV, although not statistically significant $(\mathrm{p}=0.618$ and 0.416 , respectively). For the interval T6- T18, remission rates were similar for both drugs $(\mathrm{p}=0.929)$.

Table 2 shows the most frequently reported adverse events per assessment point. The most prevalent adverse events for LEV were activating mood disorders and tiredness. Some patients experienced a positive effect on their behaviour due to LEV use. For TPM, mental slowing and dysphasia were the most important side effects at each assessment point, except for the 24-month assessment point.

The most frequently reported side effects in patients who discontinued treatment are listed in Table 3. For LEV, more than a quarter of patients (26.9\%) reported mood disorders at the time of discontinuation, with an equal distribution of activating and sedating mood disorders. Tiredness and sleepiness were also important side effects at time of discontinuation (13.8 and $8.5 \%$, respectively). In patients on TPM, mood disorders were also frequently reported (18.9\%), although sedating mood disorders played a less important role. Side effects other than mood disorders that most often led to discontinuation of TPM treatment were mental slowing (27.8\%) and dysphasia (15.0\%). Other side effects that were frequently reported were gastrointestinal complaints, paresthesia, appetite loss, skin complaints, weight loss, headache and dizziness.

Table 4 shows seizure reduction in patients who discontinued treatment. One patient $(0.8 \%)$ was seizure-free when discontinuing LEV compared to four patients (1.8\%) who were seizure-free on TPM.

In spite of the anticonvulsive effect of drug treatment, twice as many patients discontinued TPM.

More patients on LEV experienced no efficacy or even a higher seizure frequency compared to patients on TPM. 
Table 2 Most frequently reported side effects ${ }^{\mathrm{a}}$.

\begin{tabular}{|c|c|c|}
\hline & Levetiracetam & Topiramate \\
\hline \multirow[t]{3}{*}{6 Months } & Mood disorders $\uparrow^{\mathrm{b}}(8.1 \%)$ & Mental slowing (13.8\%) \\
\hline & Tiredness $(7.4 \%)$ & Dysphasiac $^{c}(6.6 \%)$ \\
\hline & Positive behaviour ${ }^{\mathrm{d}}(7.4 \%)$ & Weight loss (5.2\%) \\
\hline \multirow[t]{2}{*}{12 Months } & Mood disorders $\uparrow(5.2 \%)$ & Mental slowing (13.6\%) \\
\hline & & Dysphasia (5.7\%) \\
\hline \multirow[t]{3}{*}{18 Months } & Mood disorders $\uparrow(10.6 \%)$ & Mental slowing (8.1\%) \\
\hline & Tiredness $(6.4 \%)$ & Dysphasia $(6.5 \%)$ \\
\hline & & Mood disorders $\uparrow(5.7 \%)$ \\
\hline 24 Months & $\begin{array}{l}\text { (Number of patients too small to } \\
\text { provide meaningful data) }\end{array}$ & Urogenital complaints ${ }^{\mathrm{e}}(5.6 \%)$ \\
\hline
\end{tabular}

a Only side effects that occurred in $>5 \%$ of the patients are reported in the table; ${ }^{\mathrm{b}}$ Mood disorders $\uparrow$ : agitation, aggression, hyperirritability; ${ }^{\mathrm{c}}$ Dysphasia: word-finding difficulties; ${ }^{\mathrm{d}}$ Positive behaviour: being in a better condition; taking more initiatives; being more calm, active, cheerful, etc.; ${ }^{\mathrm{e}}$ Urogenital complaints: impotence, amenorrhea, micturation problems, etc.

Table 3 Reported side effects in patients who discontinued drug treatment ${ }^{\mathrm{a}}$.

\begin{tabular}{|c|c|}
\hline Levetiracetam & Topiramate \\
\hline Mood disorders $\uparrow^{\mathrm{b}}(13.8 \%)$ & Mental slowing $(27.8 \%)$ \\
\hline Tiredness (13.8\%) & $\operatorname{Dysphasia}^{\mathrm{c}}(15.0 \%)$ \\
\hline Mood disorders $\downarrow^{\mathrm{d}}(13.1 \%)$ & Mood disorders $\uparrow(13.2 \%)$ \\
\hline \multirow[t]{8}{*}{ Sleepiness $(8.5 \%)$} & Gastrointestinal complaints (10.6\%) \\
\hline & Paresthesia $(7.5 \%)$ \\
\hline & Appetite loss (7.0\%) \\
\hline & Skin complaints $(6.6 \%)$ \\
\hline & Weight loss $(6.2 \%)$ \\
\hline & Mood disorders $\downarrow$ (5.7\%) \\
\hline & Headache $(5.7 \%)$ \\
\hline & Dizziness (5.3\%) \\
\hline
\end{tabular}

a Only side effects that occurred in $>5 \%$ of the patients are reported in the table; ${ }^{\mathrm{b}}$ Mood disorders $\uparrow$ : agitation, aggression, hyperirritability; ' Dysphasia: word-finding difficulties; ${ }^{d}$ Mood disorders $\downarrow$ : depression, apathy.

Table 4 Seizure reduction in patients who discontinued drug treatment; follow-up period 24 months.

\begin{tabular}{lrllll}
\hline & \multicolumn{2}{c}{ Levetiracetam } & \multicolumn{2}{c}{ Topiramate } \\
\hline Seizure free & 1 & $(0.8 \%)$ & 4 & $(1.8 \%)$ \\
Lower seizure frequency & 6 & $(4.7 \%)$ & 19 & $(8.6 \%)$ \\
No efficacy & 69 & $(53.5 \%)$ & 75 & $(33.8 \%)$ \\
Higher seizure frequency & 30 & $(23.3 \%)$ & 66 & $(29.7 \%)$ \\
Not reported & 23 & $(17.7 \%)$ & 58 & $(26.1 \%)$ \\
\hline
\end{tabular}




\section{I S C USS I O N}

While many studies have been done on each individual new AED as add-on therapy, few compare these new AEDs with each other. Especially large multicenter randomized controlled trials that compare two or more AEDs are rare but crucial in clinical decision-making. Pharmaceutical companies have serious reservations funding these trials, unless there was confidence that the sponsor's drug was very likely to prove superior to the comparator. In the absence of such comparative clinical trials, indirect comparisons have been made using meta-analyses of controlled trials and reviews of long-term open-label studies to adequately evaluate the benefits and risks of treatment of epilepsy. The relevance of findings derived from such studies to everyday clinical practice is limited by several factors, especially by the indirect character of the comparisons due to differences between studies in study design, study population and placebo-effect. ${ }^{21,22}$ However, both methods have gone some way to compare new AEDs and provide some evidence of the relative efficacy and tolerability of competing new AEDs. Moreover, observational studies are known to be useful adjuncts to controlled trials to see whether the demonstrated efficacy and safety translates into effective and safe treatment in routine clinical practice. ${ }^{23}$

Our study directly compared the use of TPM with LEV, presently two of the most commonly prescribed new AEDs in chronic epilepsy. The study population consisted of patients with chronic refractory epilepsy referred to a single tertiary epilepsy centre. The added value of this study is due to the head-to-head comparison made between TPM and LEV in a single epilepsy centre, the large number of patients included ( $\mathrm{n}$ $=301$ for LEV; $\mathrm{n}=429$ for TPM), the long period of evaluation ( 24 months) and the low risk of selection bias by the inclusion of all patients who were treated with LEV or TPM in a certain period. This is illustrated by the characteristics of our study population, specifically by a wide age range and a large number of children and mentally retarded. The patients on LEV did not differ on any of the clinical or demographical variables when compared to the patients on TPM, which allows us to compare TPM to LEV and to assume that differences in outcome are drug-related.

For clinical decision-making, retention rate is a good indicator of the long-term performance of a new AED. ${ }^{24}$ It measures all possible reasons for drug discontinuation, including ineffectiveness and intolerability. In addition, it expresses the consent of an individual patient to continue drug treatment, sometimes even with side effects. Therefore, the main purpose of our study was to determine long-term retention rates for TPM and LEV. Retention rates were 65.6 and $45.8 \%$ for LEV and 51.7 and 38.3\% for TPM after 1 and 2 years, respectively. Other studies showed similar 1-year retention rates ranging from 60 to $75 \%$ for LEV and 40 to $60 \%$ for TPM. ${ }^{21}$ The statistically significant difference in 1- and 2-year retention rates is probably the consequence of a more unfavourable safety profile of TPM. At 6 months follow-up, no significant 
difference was seen between both drugs. This implies that it is hard to show differences in discontinuation rates between TPM and LEV in short-term trials, even when a large number of patients are investigated.

Several findings with regard to retention rates were noteworthy in our study. Retention rates could have been influenced by the sequence in which both drugs were marketed. Patients on TPM could have been withdrawn from treatment because of the availability of the new AED LEV. On the other hand, LEV could have been tested in a more refractory population, since LEV was introduced onto the market years after TPM.

Interestingly, the difference between continuation rates for both drugs became smaller after 1- year follow-up. We do not know whether there will still be a significant difference after many years. This is an important issue, since patients with refractory epilepsy often need lifetime treatment. One study that compared the long-term retention rates of new AEDs in a residential community of adults with chronic epilepsy showed a similar difference in 2-year retention rates between TPM and LEV compared with our study. However, this difference almost disappeared after 5 years. ${ }^{25}$

Elsewhere, our study group showed 1-, 2-, 3- and 4-year retention rates for TPM to be $53,45,38$ and $30 \%$, respectively. ${ }^{26}$ These data suggest that surviving the early stage of TPM introduction is a good indicator of long-term retention, with most withdrawals occurring in the first year. With LEV use, we have shown a similar pattern with most patients also discontinuing treatment in the first year, after which the number of withdrawals more or less remained steady at a lower rate.

Drugs that were mostly encountered as concomitant therapy were carbamazepine, clobazam, phenytoin, lamotrigine, oxcarbazepine and valproic acid. At baseline, no differences in the most frequently used concomitant AEDs between TPM and LEV could be identified. Since both drugs were prescribed as adjunctive treatment to an existing regime, we assume that similar baseline concomitant AEDs characteristics prolonged during followup. However, if not so, differences in concomitant AEDs between TPM and LEV could have influenced the efficacy and safety profile of each individual drug.

In our study, percentages of patients discontinuing drug treatment because of lack of efficacy were similar for both study groups, suggesting TPM and LEV to be equally effective. A meta-analysis of well-controlled trials showed comparable efficacy between both drugs using a responder rate of $\geq 50 \%$ reduction in seizure frequency. ${ }^{22}$ Zaccara et al. who reviewed long-term open-label studies used seizurefree outcomes as a measure of efficacy. LEV turned out to be the most effective agent, followed by TPM. However, analysis limited to prospective studies showed that TPM had the highest percentage of seizure-free patients. ${ }^{21}$ Main objective in the treatment of epilepsy is control of seizures. The achievement of seizure freedom is an essential outcome measure, because it has the greatest impact on quality of $\operatorname{life}^{27}$ and it is the 
efficacy measure that is subject to the least subjectivity. Responder rates, defined as a percentage reduction in seizure frequency, do not take into account the duration and severity of seizures. In addition, data reflecting the number of seizures are often unreliable, especially in retrospective studies. Therefore, our study only presented data regarding seizure freedom rates, although seizure remission is not a realistic goal for every patient with refractory epilepsy.

Comparable percentages of patients on TPM and LEV achieved seizure freedom for 6 months in our study. Between 11.6 and $20.0 \%$ of patients on TPM were seizurefree compared to $11.1-14.3 \%$ of patients on LEV. These figures compare well to those presented in literature, although seizure freedom rates vary a lot among different studies. Four to twenty-three percent of patients were reported to be free of seizures on TPM compared to $9.8-26.0 \%$ of patients on LEV, dependent on study design and study population. ${ }^{21}$ The proportion of patients with complete seizure control for longer periods of time provides even more clinically meaningful data for predicting the long-term efficacy of an AED. ${ }^{23}$ Therefore, we have also investigated long-term seizure remission. From baseline, more patients on LEV achieved seizure remission for at least 1 year, although this difference was not statistically significant. Possibly, this difference is due to a rapid onset of action of LEV.$^{28}$ However, if the titration period is not taken into account (i.e. in the interval T6-T18), prolonged seizure remission was achieved in more than $12 \%$ of patients in both the TPM and LEV study group, which is a good result in this highly refractory population.

As a marker of tolerability, we used the percentage of patients withdrawing drug treatment because of adverse events. Our study shows that significantly more patients discontinued TPM treatment because of adverse events than patients on LEV. This is also illustrated by the time period in which adverse events were the main reason for drug discontinuation; only in the first 3 months side effects played a more important role than lack of efficacy with LEV use compared to the first 18 months in patients on TPM. Titration rates are unlikely to be responsible for the high incidence of adverse events in patients on TPM. ${ }^{26}$ Thus, TPM was by far the least well-tolerated drug. This finding is supported by other studies reporting that TPM has a higher rate of adverse events leading to withdrawal than $\mathrm{LEV}^{16,21,22}$ and by our data showing that twice as many patients on TPM discontinued treatment despite the beneficial effects on seizure frequency compared to LEV. In these patients, the adverse events can therefore be considered very serious.

Side effect profiles of TPM and LEV are different; neurocognitive complaints are very common in TPM treatment and frequently led to drug withdrawal, while the impact of LEV on cognitive function is only very mild. One study showed that the neurocognitive effects of TPM occurred already at an early stage, while the remaining patients do not have such problems. ${ }^{29}$ However, in our study, the percentage of patients with neurocognitive side effects due to TPM use remained high. Mood disorders 
were reported in both drugs, but was the most common reason for discontinuation in patients on LEV. This contributes to clinical reports that behavioural adverse events are more common in LEV than in $\mathrm{TPM}^{30}$ and that patients taking LEV experience more behavioural symptoms than reported in RCTs. ${ }^{31}$ In our study, mood disorders in patients on LEV emerged from the beginning. It is therefore not clear why RCTs only reported somnolence, asthenia, headache and dizziness as major side effects. An explanation could be that patients with learning disabilities are more prone to behavioural side effects. ${ }^{31}$ This population is often excluded in well-controlled randomized clinical trials and some large scale open-label multicenter trials with reports of behavioural adverse events only occurring at a low rate. ${ }^{32,33}$ In conclusion, the retention rate for LEV is significantly higher than for TPM. LEV had a more favourable side effect profile than TPM with comparable efficacy. Patients on TPM discontinued treatment mainly because of neurocognitive side effects. In the treatment with LEV, the effects on mood must not be underestimated. 


\section{R E FER E NCES}

1. Stefan H, Krämer G, Mamoli B, editors. Challenge epilepsy: new antiepileptic drugs. Berlin: Blackwell Science; 1998 .

2. Cereghino JJ, Biton V, Abou-Khalil B, Dreifuss F, Gauer LJ, Leppik I. Levetiracetam for partial seizures: results of a double-blind, randomized clinical trial. Neurology 2000;55: 236-42.

3. Ben-Menachem E, Falter U. Efficacy and tolerability of levetiracetam $3000 \mathrm{mg} / \mathrm{d}$ in patients with refractory partial seizures: a multicenter, double-blind, responder-selected study evaluating monotherapy. European Levetiracetam Study Group. Epilepsia 2000;41:1276-83.

4. Multicenter double-blind, randomized placebocontrolled trial of levetiracetam as add-on therapy in patients with refractory partial seizures. European Levetiracetam Study Group. Epilepsia 2000;41:1179-86.

5. Faught E, Wilder BJ, Ramsay BJ, Reife A, Kramer LD, Pledger GW, et al. Topiramate placebo-controlled dose-ranging trial in refractory partial epilepsy using 200-, 400- and 600-mg daily dosages. Topiramate YD Study Group. Neurology 1996;46:1684-90.

6. Tassinari CA, Michelucci R, Chauvel P, Chodkiewicz J, Shorvon S, Henriksen O, et al. Double-blind, placebo-controlled trial of topiramate (600 mg daily) for the treatment of refractory partial epilepsy. Epilepsia 1996;37: 763-8.

7. Ben-Menachem E, Henriksen O, Dam M, Mikkelsen M, Schmidt D, Reid S, et al. Double-blind, placebo-controlled trial of topiramate as add-on therapy in patients with refractory partial seizures. Epilepsia 1996;37:539-43.

8. Sharief M, Viteri C, Ben-Menachem E, Weber M, Reife R, Pledger G, et al. Double-blind, placebocontrolled study of topiramate in patients with refractory partial epilepsy. Epilepsy Res 1996;25:21724.

9. Privitera M, Fincham R, Penry J, Reife R, Kramer L, Pledger G, et al. Topiramate placebo-controlled dose-ranging trial in refractory partial epilepsy using 600-, 800- and 1000-mg daily dosages. Topiramate YE Study Group. Neurology 1996;46: 1678-83.

10. Bergey GK. Evidence-based treatment of idiopathic generalized epilepsies with new antiepileptic drugs. . Epilepsia 2005;46(Suppl. 9):161-8.

11. Privitera M. Efficacy of levetiracetam: a review of three pivotal clinical trials. Epilepsia 2001;42(Suppl. 4):31-5.

12. Reife R, Pledger G, Wu S. Topiramate as add-on therapy: pooled analysis of randomized controlled trials in adults. Epilepsia 2000;41(Suppl. 1):66-71.

13. Walker MC, Sander JWAS. Difficulties in extrapolating from clinical trial data to clinical practice: the case of antiepileptic drugs. Neurology 1997;49:333-7.

14. Lhatoo SD, Wong ICK, Sander JWAS. Prognostic factors affecting long-term retention of topiramate in patients with chronic epilepsy. Epilepsia 2000;41:338-41.

15. Chung S, Wang N, Hank N. Comparative retention rates and long-term tolerability of new antiepileptic drugs. Seizure 2007;16:296-304.

16. Nicolson A, Lewis SA, Smith DF. A prospective analysis of the outcome of levetiracetam in clinical practice. Neurology 2004;63:568-70. 
17. Abou-Khalil B. Topiramate in the long-term management of refractory epilepsy. Topiramate YOL Study Group. Epilepsia 2000;41(Suppl. 1):72-6.

18. Ketter TA, Post RM, Theodore WH. Positive and negative psychiatric effects of antiepileptic drugs in patients with seizure disorders. Neurology 1999;53(Suppl. 2):S53-67.

19. Roberts GMP, Majoie HJM, Leenen LAM, Bootsma HP, Kessels AGH, Aldenkamp AP, et al. Ketter's hypothesis of the mood effects of antiepileptic drugs coupled to the mechanism of action of topiramate and levetiracetam. Epilepsy Behav 2005;6:366-72.

20. Kaplan EL. Non-parametric estimation from incomplete observations. Am Stat Assoc J 1958;53:457-81.

21. Zaccara G, Messori A, Cincotta M, Burchini G. Comparison of the efficacy and tolerability of new antiepileptic drugs: what can we learn from long-term studies? Acta Neurol Scand 2006;114:157-68.

22. Otoul C, Arrigo C, Van Rijckevorsel K, French JA. Meta analysis and indirect comparisons of levetiracetam with other second-generation antiepileptic drugs in partial epilepsy. Clin Neuropharmacol 2005;28:72-8.

23. Mohanraj R, Brodie MJ. Measuring the efficacy of antiepileptic drugs. Seizure 2003;12:413-43.

24. Sander JW. New antiepileptic drugs in practice-how do they perform in the real world? Acta Neurol Scand 2005;112 (Suppl. 181):26-9.

25. Simister RJ, Sander JW, Koepp MJ. Long-term retention rates of new antiepileptic drugs in adults with chronic epilepsy and learning disability. Epilepsy Behav 2007;10:336-9.

26. Bootsma HPR, Coolen F, Aldenkamp AP, Arends J, Diepman J, Hulsman J, et al. Topiramate in clinical practice: long-term experience in patients with refractory epilepsy referred to a tertiary epilepsy center. Epilepsy Behav 2004;5:380-7.

27. Walker MC, Sander JW. The impact of new antiepileptic drugs on the prognosis of epilepsy: seizure freedom should be the ultimate goal. Neurology 1996;46:912-4.

28. Specchio LM, Boero G, Specchio N, De Agazio G, De Palo A, de Tommaso M, et al. Evidence for a rapid action of levetiracetam compared to topiramate in refractory partial epilepsy. Seizure 2006;15:112-6.

29. Tatum WO, French JA, Faught E, Morris GL, Liporace J, Kanner A, et al. Postmarketing experience with topiramate and cognition. Epilepsia 2001;42:1134-40.

30. Weintraub D, Buchsbaum R, Resor Jr SR, Hirsch LJ. Psychiatric and behavioural side effects of the newer antiepileptic drugs in adults with epilepsy. Epilepsy Behav 2007;10:105-10.

31. White JR, Walczak TS, Leppik IE, Rarick J, Tran T, Beniak TE, et al. Discontinuation of levetiracetam because of behavioral side effects: a case-control study. Neurology 2003;61:1218-21.

32. Morrell MJ, Leppik I, French J, Ferrendelli J, Han J, Magnus L. The KEEPER trial: levetiracetam adjunctive treatment of partial-onset seizures in an open-label community-based study. Epilepsy Res 2003;54:153-61.

33. Lambrechts DAJE, Sadzot B, Van Paesschen W, van Leusden JA, Carpay J, Bourgeois P, et al. Efficacy and safety of levetiracetam in clinical practice: results of the SKATE trial from Belgium and The Netherlands. Seizure 2006;15:434-42. 



\section{seven}

\section{The effect of anti-epileptic drugs on cognition: patient perceived cognitive problems of topiramate versus levetiracetam in clinical practice}




\section{A B S T R A C T}

Introduction: Neurocognitive complaints may interfere with long-term antiepileptic drug (AED) treatment and are an important issue in clinical practice. Most data about drug-induced cognitive problems are derived from highly controlled short-term clinical trials. We analyzed such cognitive complaints for the two most commonly used AEDs in a clinical setting using patient perceived problems as primary outcome measure.

Method: All patients of the epilepsy center Kempenhaeghe that received topiramate (TPM) or levetiracetam (LEV) from the introduction to mid 2004 were analyzed using a medical information system, an automated medical file. Patients were analyzed after 6,12 , and 18 months of treatment.

Results: Four hundred and two patients used either TPM $(\mathrm{n}=260)$ or LEV ( $\mathrm{n}=142)$; 18 months retention showed a statistically significant difference, revealing $15 \%$ more patients that continued LEV compared to TPM: 18 months retention $46 \%$ for TPM and $61 \%$ for LEV $[\mathrm{F}(1.400)=3.313, \mathrm{p}=0.043]$.

Neurocognitive complaints accounted for a significant number of drug discontinuations and especially the high frequency of neurocognitive complaints in the first period of TPM treatment appeared to be significant different from LEV $[\mathrm{F}(2,547)$ $=3.192, \mathrm{p}=0.042 \mathrm{]}$. In the remaining patients, the difference in neurocognitive complaints was not statistically significant.

Conclusion: cognitive complaints are common in TPM treatment and frequently lead to drug withdrawal. The impact of LEV on cognitive function is only mild. This leads to a much higher (15\%) drug discontinuation rate for TPM compared to LEV. 


\section{N T ROD UC T I O N}

Many of the patients with refractory epilepsy may benefit from the introduction of newer antiepileptic drugs (AEDs) in clinical practice ${ }^{1}$. Two of the more commonly used newer AEDs are topiramate (TPM) and levetiracetam (LEV). TPM, a sulfamated monosaccharide, has multiple mechanisms of action ${ }^{2}$ and was proven to be effective in patients with refractory chronic partial epilepsies in shortterm controlled clinical trials ${ }^{3}$. Previous studies have clearly shown that treatment with TPM is associated with all kinds of side effects. The CNS-related side effects, and especially the neurocognitive effects ${ }^{4-6}$, are among the most frequent reported and have the most serious impacts on daily-life-function ${ }^{7}$. Most of the side-effects reports come from the clinical trials and thus represent the adverse effects of the drugs at short term and in relatively controlled circumstances ${ }^{8}$. It is not clear whether the tolerability problems persist at long term, although some evidence exists that habituation for TPM occurs in a later stage than for most of the AEDs ${ }^{4,5}$.

LEV (ucb L059) has been approved as adjunctive therapy in the treatment of partial onset seizures in adults with epilepsy. The mode of action does not involve direct interaction with known mechanisms of inhibitory or excitatory neurotransmission. Studies in animals have demonstrated that LEV and the major metabolite ucb L057 present a low risk of toxicity, and LEV is unlikely to interact with other drugs ${ }^{9}$. In the available studies, the most frequently reported adverse events associated with the use of LEV, which were not seen at an equivalent frequency among placebo-treated patients, were somnolence, asthenia, infection, and dizziness, however, at low incidence $^{10}$.

In the regulatory trials, TPM is thus characterized with neurocognitive effects, whereas these effects seem to be absent in LEV. Some evidence exists that these effects are subject to habituation in long-term TPM treatment, whereas the long-term effects of LEV have not yet been reported.

This illustrates an important point and focus of our study. For patients with refractory epilepsy, the value of a drug in clinical practice cannot be simply derived from the outcomes of short-term trials. For example, a mere $11 \%$ of the patients discontinued treatment in most of the controlled clinical trials, whereas this seems to be much higher in clinical practice ${ }^{11}$. Three-year retention for example was in the range of $40 \%$ for TPM, lamotrigine (LTG), and gabapentin (GBP). This implies that about $60 \%$ of the patients will discontinue treatment within a period of 3 years. The reason to discontinue treatment was because of tolerability problems in about half of the withdrawals, i.e., about $30 \%$. From previous studies it is expected that neurocognitive problems are one of the most commonly reported tolerability problems ${ }^{12}$ and their evaluation during long-term treatment is thus pertinent. 
As yet, the long-term cognitive profile of TPM and LEV is not available for clinical decision making. For our specific patient group (i.e., patients with refractory epilepsies in a tertiary epilepsy referral and care center) such data become increasingly more important as more drugs and nonpharmacological options become available. We therefore performed a systematical audit of TPM and LEV use in the "real life" setting of our center, analyzing all patients that received or are still using TPM or LEV.

\section{METHODS}

The study was approved by the Medical Ethics Committee. All patients who had ever been treated with TPM or LEV in the epilepsy center Kempenhaeghe from the introduction of the drug in spring 1993 and 1999 (TPM and LEV, respectively) up to a final assessment point middle of 2004 were identified by means of our automated Medical Information System (MIS) and subsequently analyzed.

Only patients that used the drug for at least 6 months were included. This latter criterion was used because our focus on cognitive complaints. Cognitive side effects generally develop gradually and are characterized as a chronic side effect. The method of analysis has been described by our research group elsewhere ${ }^{13}$.

Period of treatment was evaluated each 6 months for a total of 18 months. Data forms were entered in a computerized database for analysis. Statistical analysis was performed using SPSS 10.0 for Windows. Nonparametric variables were analyzed using chi square test, whereas parametric data were analyzed with analysis of variance (ANOVA).

\section{R E S U L T S}

Table 1 shows the main characteristics of the patients: 402 patients were identified, 260 using TPM and 142 using LEV. Gender is equally distributed in both groups and about two-thirds of the patients are in the adult age in both groups. Age at onset is in the 10th and 11th year, respectively, and both groups can be characterized as refractory as they both have patients with an average $>20$ years of active epilepsy (=years with seizures). In line with this, most patients have a cryptogenic or symptomatic localization-related epilepsy with partial seizures with or without secondary generalization; distribution of epilepsy and seizure types is similar for the two groups. About one third of the patients are mentally challenged. All comparisons between the groups show no statistically significant differences: gender $\left(\chi^{2}=0.102, p=0.75\right)$, age $\left(\chi^{2}=0.131, p=0.718\right)$, type of epilepsy $\left(\chi^{2}=11.574, p=0.072\right)$, seizure type $\left(\chi^{2}=7.784\right.$, 
Table 1 Patient demographics and characteristics.

\begin{tabular}{|c|c|c|}
\hline & Topiramate $(\mathrm{N}=260)$ & Levetiracetam $(\mathrm{N}=142)$ \\
\hline Gender: male/female & $127 \mathrm{M} / 133 \mathrm{~F}$ & $67 \mathrm{M} / 75 \mathrm{~F}$ \\
\hline Percentage (\%) & $49 \% / 51 \%$ & $47 \% / 53 \%$ \\
\hline Age: Child $\leq 12$ years/adults & $59 / 201$ & $30 / 112$ \\
\hline Percentage (\%) & $23 \% / 77 \%$ & $21 \% / 79 \%$ \\
\hline Age at onset (SD) & 10.47 years $(11.35)$ & 11.86 years $(10.92)$ \\
\hline $\begin{array}{l}\text { Duration of 'active epilepsy' } \\
\text { (years with seizures (SD) }\end{array}$ & 20.60 years $(12.25)$ & 21.04 years $(12.33)$ \\
\hline \multicolumn{3}{|l|}{ Type of epilepsy } \\
\hline Localization-related idiopathic & $3(1 \%)$ & $2(1 \%)$ \\
\hline Localization-related symptomatic & $85(33 \%)$ & $49(35 \%)$ \\
\hline Localisation-related cryptogenic & $121(46 \%)$ & $48 \quad(34 \%)$ \\
\hline Gegeneralized idiopathic & $7(3 \%)$ & $7(5 \%)$ \\
\hline Gegeneralized symptomatic & $16(6 \%)$ & $12(8 \%)$ \\
\hline Gegeneralized cryptogenic & $16(6 \%)$ & $8(6 \%)$ \\
\hline Unclassified or specific syndromes & $12(5 \%)$ & $16(11 \%)$ \\
\hline \multicolumn{3}{|l|}{ Seizure types } \\
\hline Partial seizures & $147(57 \%)$ & $71 \quad(50 \%)$ \\
\hline Gegeneralized seizures & $35(14 \%)$ & $26(18 \%)$ \\
\hline Partial secondary generalized seizures & $71(27 \%)$ & $34(24 \%)$ \\
\hline Unclassified & $7(3 \%)$ & $11(8 \%)$ \\
\hline Mental handicap: yes/no & $78 / 182$ & $44 / 98$ \\
\hline Percentage (\%) & $30 \% / 70 \%$ & $31 \% / 69 \%$ \\
\hline Mean starting dose & $26.4 \mathrm{mg} /$ day & $602.5 \mathrm{mg} /$ day \\
\hline Mean titration dose/week & $23.5 \mathrm{mg}$ & $443.6 \mathrm{mg}$ \\
\hline Mean clinical dose (at 6 months) & $217.3 \mathrm{mg} /$ day & $1951.5 \mathrm{mg} /$ day \\
\hline
\end{tabular}

$\mathrm{p}=0.061)$, mental handicap $\left(\chi^{2}=0.042, \mathrm{p}=0.837\right)$ age at onset $[\mathrm{F}(1,396)=1.382, \mathrm{p}=$ $0.241]$ duration of epilepsy $[\mathrm{F}(1.386)=.117, \mathrm{p}=0.732]$. The two groups are therefore similar for most important clinical and demographic variables.

Figure 1 shows the 18 months retention for TPM and LEV in our patient group. There is a significant difference $[\mathrm{F}(1.400)=3.313, \mathrm{p}=0.043]$ only for the 18 months point, with $15 \%$ more patients discontinuing treatment for the TPM group (46\% of patients on TPM still on treatment after 18 months vs. $61 \%$ on LEV). The $100 \%$ retention rate at 6 months is the consequence of our inclusion criteria (i.e., only patients were included that used the drug at least 6 months).

Table 2 and Figure 1 show that the percentage of patients with cognitive complaints is only higher (for TPM) in the first assessment after 6 months (statistical significant 


\section{Chapter $\mid$ seven}

Table 2 Cognitive complaints for topiramate versus levetiracetam.

\begin{tabular}{llll}
\hline & 6 months & $12 \mathrm{months}$ & $18 \mathrm{months}$ \\
\hline Cognitive complaints & $40 \mathrm{patients}(\mathrm{pt}) / 6 \mathrm{pt}$ & $14 \mathrm{pt} / 9 \mathrm{pt}$ & $12 \mathrm{pt} / 4 \mathrm{pt}$ \\
(TPM/LEV) & $15 \% / 4 \%$ & $9 \% / 9 \%$ & $9 \% / 5 \%$ \\
p-values & $\mathrm{P}=0.042$ & $\mathrm{Ns}$ & $\mathrm{Ns}$ \\
& & & \\
1 area of cognitive complaints & $32 \mathrm{pt} / 6 \mathrm{pt}$ & $13 \mathrm{pt} / 9 \mathrm{pt}$ & $11 \mathrm{pt} / 4 \mathrm{pt}$ \\
(TPM/LEV) & $12 \% / 4 \%$ & $8 \% / 9 \%$ & $9 \% / 5 \%$ \\
2 or more areas of cognitive & $8 \mathrm{pt} / 0 \mathrm{pt}$ & $1 \mathrm{pt} / 0 \mathrm{pt}$ & $1 \mathrm{pt} / 0 \mathrm{pt}$ \\
complaints (TPM/LEV) & $3 \% / 0 \%$ & $1 \% / 0 \%$ & $0 \% / 0 \%$ \\
Total patients & $260 / 142 \mathrm{pt}$ & $162 / 98 \mathrm{pt}$ & $128 / 86 \mathrm{pt}$ \\
\hline
\end{tabular}

Only the percentage of patients with complaints is tested for statistical significance. Ns, not significant.

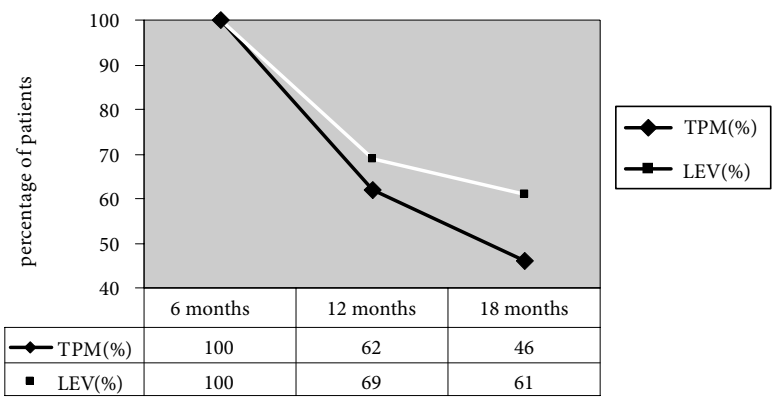

Figure 1 Long-term retention (\% of patients that continue treatment) for topiramate versus levetiracetam.

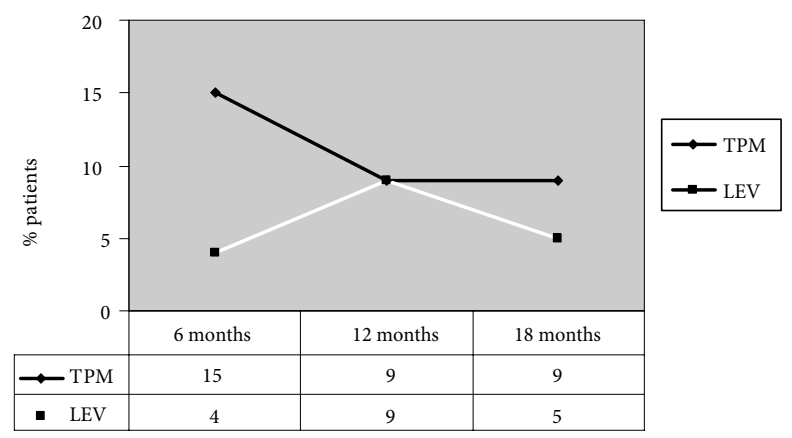

Figure 2 Cognitive complaints for topiramate and levetiracetam. 
Table 3 Cognitive complaints for topiramate versus levetiracetam only for patients that continue treatment over the 18 months follow-up.

\begin{tabular}{llll}
\hline & 6 months & 12 months & 18 months \\
\hline $\begin{array}{l}\text { Number of patients that continue drug } \\
\text { treatment over } 18 \text { months (TPM/LEV) }\end{array}$ & 116 versus 82 & 116 versus 82 & 116 versus 82 \\
$\begin{array}{l}\text { \% of patients (pt) with cognitive } \\
\text { complaints (TPM/LEV) }\end{array}$ & $10 \mathrm{pt} / 4 \mathrm{pt}$ & $9 \mathrm{pt} / 5 \mathrm{pt}$ & $12 \mathrm{pt} / 4 \mathrm{pt}$ \\
\hline
\end{tabular}

$[\mathrm{F}(2,547)=3.192, \mathrm{p}=0.042]$. In the other assessment points (at 12 and 18 months, the percentage are comparable. We have to take into account here the survival rate: the number of patients at the 12th and 18th months are lower than at the 6th month (see Figure 2). Possibly, the data here reflect a kind of "survival profile": those patients on TPM that do not tolerate the drug because of its neurocognitive effects discontinue treatment while the profiles are not different in the remaining "survivors." In line with this finding, the results show that if the analyses are repeated only for the patients still on the drug for 18 months, then no difference is found between TPM and LEV, also not at the 6 -months point $[\mathrm{F}(1,197)=1.603, \mathrm{p}=0.207]$. Table 3 illustrates these findings.

Finally for all patients that discontinued treatment the actual reason for drug withdrawal was recorded. This shows that at the 6 months point $24 \%$ of the TPM versus $6 \%$ of the LEV withdrawals were due to the neurocognitive complaints; this was $18 \%$ versus $13 \%$ for the 12 months follow-up and $14 \%$ versus $0 \%$ for the 18 months followup. To test for statistical significance, a 1-factor ANOVA was performed, showing that only the difference between TPM and LEV was significant at the 6-months point [6-months: $\mathrm{F}(1,113)=5.466, \mathrm{p}=0.021 ; 12$-months $\mathrm{F}(1,54)=0.205, \mathrm{p}=0.653$; 18-months: $\mathrm{F}(1,30)=1.985, \mathrm{p}=0.169$ ]. So although the number of patients on TPM that stop because of neurocognitive problems remain high, this is only statistically significant different at the 6-months point.

\section{I S C U S S I O N}

The added value of this study is due to the large number of patients included ( $n=402)$, the long period of evaluation (18 months) and the unselected group (i.e., all patients that were given TPM and LEV treatment from introduction to a recent period in one specialized center).

Moreover, the patients on TPM did not differ on any of the clinical or demographical variables when compared with the patients on LEV. We may therefore assume that the complaints are drug-related. This study shows the importance of complaints 
(so subjectively perceived problems) in the neurocognitive area. For TPM, 24\% of the drug withdrawals after 6 months of treatment are due to such neurocognitive complaints. This means that the cognitive side-effects must not be underestimated in clinical practice.

The expected difference between TPM and LEV in terms of impact on the neurocognitive system is confirmed in this study: patients on TPM have significantly more complaints about drug-induced cognitive impact than patients on LEV. However, this difference is only statistically significant at short term, i.e., at the 6-months evaluation point. The complaints contribute significantly to drug discontinuation at this point $(24 \%$ of the withdrawals at 6 months due to the neurocognitive complaints alone and possible a higher percentage in whom such complaints played a role in drug discontinuation). Therefore, only patients that tolerated the drug (i.e., were not sensitive for such cognitive complaints) continued. This contributes to the clinical report that some patients do not tolerate the neurocognitive effects of this drug and that these problems occur already at an early stage, while the remaining patients do not have such problems. Nonetheless, the percentage of cognitive complaints for TPM remained high (i.e., about $18 \%$ and $14 \%$ of the TPM drug withdrawals were due to neurocognitive problems at the 12- and 18-months evaluations. This factor significantly contributed to the lower retention rate for TPM in comparison to LEV.

At the 18 -month evaluation, $46 \%$ of the patients still used TPM (i.e., $54 \%$ discontinued treatment), whereas $61 \%$ were still on LEV. Patients on LEV are not completely without neurocognitive complaints, but the cognitive impact of this drug is mild compared to TPM.

In conclusion, cognitive complaints are common in TPM treatment and frequently lead to drug withdrawal. The impact of LEV on cognitive function is only mild. This leads to a much higher (15\%) drug discontinuation rate for TPM compared to LEV. 


\section{R E F E R E N C E S}

1. Stefan H, Krämer G, Mamoli B.(eds). Challenge epilepsy - new antiepileptic drugs. Berlin: Blackwell Science, 1998.

2. White HS. Clinical significance of animal seizure models and mechanism of action studies of potential antiepileptic drugs. Epilepsia 1997;38(suppl. 1):S9-17.

3. Faught E, Wilder BJ, Ramsay RE, et al. Topiramate placebo controlled dose-ranging trial in refractory partial epilepsy using 200-, 400-, 600 mg dosages. Neurology 1996;46:1684-90.

4. Aldenkamp AP. Cognitive effects of topiramate, gabapentin and lamotrigine in healthy young adults. Neurology 2000;54:270-2.

5. Aldenkamp AP, Baker G, Mulder OG, et al. A multicentre randomized clinical study to evaluate the effect on cognitive function of topiramate compared with valproate as add-on therapy to carbamazepine in patients with partial-onset seizures. Epilepsia 2000;41:1167-78.

6. Tatum WO, French JA, Faught E, et al. Postmarketing experience with topiramate and cognition. Epilepsia 2001;42:1134-40.

7. Rosenfeld WE. Topiramate: a review of preclinical pharmacokinetic and clinical data. Clin Ther 1997;19:1294-308.

8. Kellet MW, Smith DF, Stockton PA, et al. Topiramate in clinical practice: first year's postlicensing experience in a specialist epilepsy clinic. J Neurol Neurosurg Psychiatry 1999;66:759-63.

9. Harden C. Safety profile of levetiracetam. Epilepsia 2001;42(suppl. 4):36-9.

10. Krakow K, Walker M, Otoul C, et al. Long-term continuation of levetiracetam in patients with refractory epilepsy. Neurology 2001;56:1772-4.

11. Lhatoo SD, Wong ICK, Sander JWAS. Prognostic factors affecting long-term retention of topiramate in patients with chronic epilepsy. Epilepsia 2000;41:338-41.

12. Wong ICK. New antiepileptic drugs: study suggests that a quarter of patients will still be taken the new drugs after six years. BMJ 1997;314:603-4.

13. Bootsma HPR, Coolen F, Aldenkamp AP, et al. Topiramate in clinical practice: long-term experience in patients with refractory epilepsy referred to a tertiary epilepsy center. Epilepsy Behav 2004:5:380-7. 



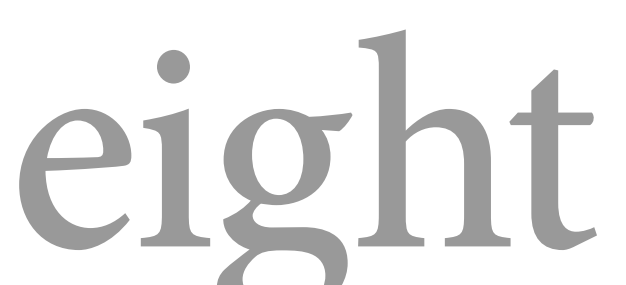

Ketter's hypothesis of the mood effects of anti-epileptic drugs coupled to the mechanism of action of topiramate and levetiracetam 


\section{A B S T R A C T}

Mood-modulating profiles of antiepileptic drugs (AEDs) have been classified by Ketter, Post, and Theodore [Neurology 1999; 53 (5, Suppl. 2) S53-76] into two classes: the first class is assumed to have deactivating effects related to GABA potentiation, and the second class is assumed to have activating effects that are associated with glutamate attenuation. We tested this hypothesis by reviewing the multiple mechanisms of action of topiramate (TPM) and levetiracetam (LEV) together with clinical behavioral side effects of patients who had been treated with TPM and LEV in a tertiary referral center for epilepsy. We found LEV to manifest activating and deactivating side effects equally and TPM to act as a deactivating AED, with tiredness/ sleepiness side effects being predominant. TPM, in comparison to LEV, was found to be associated with a high incidence of side effects. Testing the hypothesis of Ketter et al. (1999) the deactivating effects of TPM may be coupled to a predominance of potentiation of GABA, but the oversimplified basis of the model needs to be acknowledged. 


\section{N T RODUCT IO N}

AEDs that act on the brain often affect multiple brain areas and brain functions as well as those that are targeted for seizure control. For several reasons, availability of a single AED with multiple mechanisms of action may be preferable to the use of multiple AEDs. These reasons include ease of titration, reduced potential for pharmacodynamic tolerance, and avoidance of drug-drug interactions ${ }^{1}$. A single drug with multiple mechanisms of action can be desirable when medications developed as AEDs are effective in treating depression, anxiety, migraine, bipolar disorder, pain, or other central nervous system (CNS) disorders. Unfortunately all have some undesirable properties that limit their utility and complicate patient management and compliance. In many patients seizure control is achieved only at the expense of various types and degrees of severity of AED-related adverse effects. Frequently reported neurological problems include asthenia, diplopia, dizziness, fatigue, headache, somnolence, and tremor, whereas behavioral side effects include aggression, agitation, anxiety, depression, emotional hostility, hyperactivity, nervousness, and psychosis $^{2}$. The most frequently reported side effects are CNS-related side effects, and these have the most serious impact on daily life function ${ }^{3}$.

The majority of reports of side effects come from clinical trials of short duration so they represent short-term adverse effects in relatively controlled circumstances; therefore, it is unclear whether tolerability or habituation is evident over the long term. Patients with cognitive and anxiety disorders have been reported to have lower incidences of side effects ${ }^{4,5}$. Cognitive and anxiety symptoms are often even more prominent in patients with refractory epilepsy. In general, patients with refractory epilepsy require long-term treatment.

Refractory epilepsy is a difficult condition to treat, and most patients, regardless of syndrome, usually try numerous drugs in an attempt to reduce seizure frequency or become seizure free ${ }^{6}$.

All AEDs can cause similar types of CNS adverse effects despite dissimilar mechanisms of action ${ }^{4}$. Ketter et al. hypothesized that drugs with GABAergic mechanisms differed with respect to their effects on behavior from drugs with antiglutamatergic mechanisms ${ }^{7}$.

The GABAergic group has "deactivating" effects associated with fatigue, cognitive slowing, weight gain, and potential anxiolytic and antimanic effects. Conversely the antiglutamatergic group has "activating" effects associated with activation, weight loss, and potential anxiogenic and antidepressant effects. In using this classification it is essential that we take the baselines of both children and adults into account, as we want to avoid exacerbating existing problems and potentially induce favorable behaviors. However, the occurrence of various behavioral side effects during trials of 
every new AED with various mechanisms of action suggests that the problem is not specific to a particular drug mechanism.

This lack of fit may result from using a simple classification that is based only on the predominant mechanism of action to classify a drug ${ }^{8}$. Clinical evaluations of more precise relationships of mechanisms of action to behavioral profiles of AEDs are clearly needed to assess the validity of this broad proposition and identify exceptions to this bipolar hypothesis.

Topiramate (TPM) and levetiracetam (LEV) have proven to be effective in patients with chronic partial epilepsies and are the focus of this study. TPM is derived from D-fructose, a naturally occurring monosaccharide, and was developed initially as an antidiabetic drug. LEV is the S-enantiomer of a-ethyl-2-oxo-1-pyrrolidine acetamide, a piracetam analog. It was first investigated in the 1980s as a drug with cognitive enhancing and anxiolytic effects. Both drugs provide protection in a broad range of animal models of epilepsy (Table 1). TPM appears to have multiple mechanisms of action, while the mode of action of LEV is incompletely understood (Table 2).

Previous studies have clearly shown that exposure to TPM and LEV is associated with all kinds of side effects.

In patients with refractory epilepsy, eight studies ${ }^{9-16}$ have evaluated the efficacy of TPM and three studies ${ }^{17-19}$ have evaluated the efficacy of LEV as add-on therapy. In these studies the most frequently reported side effects of TPM included somnolence, fatigue, anorexia, weight loss, psychomotor slowing and confusion, and headache, while the most frequently reported side effects of LEV were dizziness, somnolence, asthenia, headache, and infection. These adverse effects are comparable to those reported in patients with nonrefractory epilepsy.

In addition to epilepsy, the molecular targets of TPM and LEV have been associated with other CNS disorders. The data are mixed regarding the acute antidepressant properties of TPM, whereas the possible antidepressant effect of LEV is just beginning to be evaluated.

A number of open clinical trials in which TPM has been used adjunctively with other agents suggest antimanic effects ${ }^{20-22}$. TPM may have potential for the treatment of depression in obese patients, but close monitoring of weight and mood effects is warranted ${ }^{23,24}$.

There has been no initial description of the positive antidepressant and pychotropic effects of LEV in patients with seizure disorders.

All AEDs have mechanisms that suppress or control seizure activity, but no two drugs have the exact same mechanism of action. Various mechanisms of action lead to diverse anticonvulsant and other side effects. It is hypothesized that differences between drugs in the side effects manifested may be attributed to differences in the multiple brain areas and brain functions that are responsible for anticonvulsant action. This article briefly discusses the currently proposed mechanisms of these 
Table 1 Effects in animal models of seizures and epilepsya.

\begin{tabular}{lll}
\hline & TPM & LEV \\
\hline Maximal electroshock seizure & $\sqrt{ }[25]$ & $\mathrm{X}[26,27]$ \\
Pentylenetetrazol & $\mathrm{X}[25,28]$ & $\mathrm{X}[26,27]$ \\
Other chemoconvulsants & $\mathrm{X}[25,28]$ & Modest activity $[29]$ \\
Genetic spontaneous epileptic rat & $\sqrt{ }[30]$ & $\sqrt{ }[29,31]$ \\
Amygdala-kindled & $\sqrt{ }[32,33]$ & $\sqrt{ }[27]$ \\
\hline
\end{tabular}

$\sqrt{ }$, active; $X$, inactive.

Table 2 Mechanisms of action of TPM and LEV.

\begin{tabular}{|c|c|c|}
\hline Target & TPM & LEV \\
\hline GABA & $\begin{array}{l}\text { Allosteric modulator of some GABA } \\
\text { receptors (subunit selective) }[1,34] \\
\text { Acts like benzodiazepine but effect is not } \\
\text { blocked by the antagonist flumazenil [35] } \\
\text { Increases GABA brain concentrations [36] } \\
\text { Carbonic anhydrase inhibition may } \\
\text { influence GABA depolarization [37] }\end{array}$ & $\begin{array}{l}\text { No consistent effect on amplitude [38] } \\
\text { Suppression of inhibition by negative } \\
\text { modulators [38,39] } \\
\text { Short lived increase in GABA brain } \\
\text { concentrations (rapid normalisation } \\
\text { questions anticonvulsant relevance) [40] }\end{array}$ \\
\hline Glutamate & Inhibits kainate and AMPA currents $[41,42]$ & Inhibits AMPA currents [43] \\
\hline $\mathrm{Na}^{+}$ & State-dependent blockade $[44,45]$ & No effect [46] \\
\hline $\mathrm{Ca}^{2+}$ & $\begin{array}{l}\text { Reduces amplitude of high but not } \\
\text { low-activated } \mathrm{Ca}^{2+} \text { currents }[47,48]\end{array}$ & $\begin{array}{l}\text { Reduces amplitude of high but not } \\
\text { low-activated } \mathrm{Ca}^{2+} \text { currents }[49] \\
\mathrm{Ca}^{2+} \text { mechanisms are hypothesized } \\
\text { to account for a non-GABA } \text {-related }_{\text {antibicuculline effect }[50,51]}\end{array}$ \\
\hline $\mathrm{K}^{+}$ & Activation of conductance [37] & Inhibition of the delayed rectifier [52] \\
\hline
\end{tabular}

drugs to offer some observations on the significance of mechanism findings in relation to behavioral/mood effects from the scientific and clinical perspectives. We refer to the "Ketter model" with great emphasis placed on the mood aspects of the model relative to the oversimplified direct link between the GABA and glutamate mechanisms of action.

\section{METHODS}

All treatment-resistant patients who had been treated with TPM and LEV in a tertiary referral center for epilepsy were identified by means of an automated medical information system (MIS). Patients were included if the start date of their medication was at least 1 year before the cutoff date of April 2004. Of the 773 patients who were identified, 6 patients were already on LEV when TPM was introduced and 16 patients were already on TPM when LEV was introduced. These patients were elimi- 
nated from the study, leaving 464 TPM patients and 247 LEV patients. Data were obtained from both individual patient records and the MIS. The following variables were entered into a database: patient demographics and characteristics (sex, age, age of onset of epilepsy, duration of epilepsy when TPM or LEV treatment commenced, mental retardation, CT/MRI abnormalities, seizure type), comedication, and side effects. Epilepsy and seizures were classified using the International League Against Epilepsy (ILAE) Classification. Side effects and comedications were clustered using a two-point yes/no scale for the purpose of this study. For the clustered side effects, a definition consensus was reached to try to avoid discrepancies between individual raters. The following side effects were investigated: activating (aggression, increased fitness, energy, alertness, hyperactive, agitated, irritated), deactivating (depressive, feeling bad, not sociable, anhedonia), tiredness/sleepiness (the two side effects were grouped together because it was not always possible to differentiate between the two problems as they were often intertwined), total deactivating (deactivating or tired/ sleepy), headache, rest of CNS, and food problems (anorexia, bulimia, gastrointestinal problems). The following medications were clustered according to their behavioral effects, but some of the drugs were not clustered due of lack of behavioral overlap as reported in the literature: carbamazepine/phenytoin/oxcarbazepine, clobazam/clonazepam/phenobarbital/heptobarbital/methylphenobarbital/primidone, gabapentin/ pregabalin/tiagabine, acetazolamide, ethosuximide, lamotrigine, valproate, vigabatrin. Time points were also clustered so that we could evaluate from the commencement of drug administration to 6 months and from 6 to 18 months inclusive. The periodic time point evaluation was every 6 months. If no visit was available at these assessment points, the nearest visit within 6 months was used. Statistical analysis was performed using SPSS 10.0 for Windows. The differences in distribution between the two groups were tested nonparametrically using Mann-Whitney U tests, and nominal data were analyzed using $\chi^{2}$ tests. Nonparametric data are expressed as means and SD. Proportions are expressed as percentages. To take into account unequal distributions of the covariables we calculated unadjusted and adjusted odds ratios to study the adjusted association between study medication and side effects. This was done with the help of a logistic regression that used side effects as the dependent variable and study medication and covariables as the independent variables.

\section{RES U L T S}

Table 3 summarizes the main demographics and characteristics of the patients: These two groups of patients on LEV and TPM represent a typical group of patients referred to a tertiary epilepsy center. Mann- Whitney $U$ analysis revealed no significant difference $(\mathrm{P}<0.05)$ with respect to age, age at onset of seizure, or duration of epilepsy before TPM/LEV introduction. 
Table 3 Patient demographics and characteristics.

\begin{tabular}{lcc}
\hline & LEV $(n=247)$ & TPM $(n=464)$ \\
\hline Age & $32.5(17.2)^{\mathrm{a}}$ & $29.7(16.1)$ \\
Age at onset of seizure & $11.8(11.6)$ & $10.2(11.5)$ \\
Duration of epilepsy before TPM/LEV introduction & $21.2(12.7)$ & $20.8(13.5)$ \\
Sex (F) \% & 45.3 & 51.9 \\
Minor seizures ${ }^{\mathrm{b}}(\%)$ & 81.8 & 80.2 \\
Major seizures (\%) & 70.9 & 72.0 \\
Unclassified seizures (\%) & 4.0 & 4.5 \\
Nonepileptic seizures (\%) & 6.9 & 6.7 \\
Mental retardation (\%) & 42 & 35 \\
CT (\%) & 39 & 44 \\
MRI (\%) & 63 & 63 \\
\hline
\end{tabular}

a Mean (SD); ${ }^{\text {b }}$ Minor seizures include simple partial, complex partial, absence and myoclonic seizures. Major seizures include partial seizures evolving into secondary generalized, tonic, tonic-clonic and atonic seizures.

Table $4 \chi^{2}$ comparison of comedications between patients on LEV and those on TPM ${ }^{\mathrm{a}}$.

\begin{tabular}{|c|c|c|c|c|}
\hline & \multicolumn{2}{|c|}{0 Months } & \multicolumn{2}{|c|}{6 Months } \\
\hline & LEV & TPM & LEV & TPM \\
\hline Acetazolamide & 2.0 & 1.1 & 1.7 & 0.4 \\
\hline Lamotrigine & 41.65 & 44.9 & 17.2 & 18.3 \\
\hline TPM/LEV & $18.5^{\star *}$ & 1.3 & 1.3 & 1.3 \\
\hline Valproate & 18.2 & 19.8 & 6.9 & 11.3 \\
\hline Vigabatrin & 3.6 & 6.2 & 3.0 & 0.4 \\
\hline $\mathrm{CFO}^{\mathrm{b}}$ & 65.3 & 67.7 & $29.4^{* *}$ & 41.9 \\
\hline Benzamidine $^{c}$ & $45.5^{\star}$ & 1.5 & $21.8^{*}$ & 28.3 \\
\hline Ethosuximide & $4.0^{*}$ & 1.5 & 1.7 & 1.1 \\
\hline $\mathrm{GABA}^{\mathrm{d}}$ & $4.6^{\star *}$ & 0.6 & 0.7 & $0.2^{\mathrm{a}}$ \\
\hline
\end{tabular}

${ }^{a}$ Results are expressed as percentages, and were analyzed using $\chi^{2}$ tests; ${ }^{b}$ Carbamazepine, phenytoin, or oxcarbazepine; ${ }^{\mathrm{C}}$ Clobazam/clonazepam/phenobarbital, heptobarbital, methylphenobarbital, or primidone; ${ }^{\mathrm{d}}$ Gabapentin, pregabalin, or tiagabine; ${ }^{*} \mathrm{P}<0.05$ vs TPM; ${ }^{* *} \mathrm{P}<0.01$ vs TPM.

$\chi^{2}$ analysis revealed no significant difference $(\mathrm{P}<0.05)$ with respect to seizure type.

At $\mathrm{t}=0$ the mean TPM dose was $44 \mathrm{mg} / \mathrm{day}$, and after titration this reached 220 $\mathrm{mg} /$ day. For LEV at $\mathrm{t}=0$, the mean dose was $670 \mathrm{mg} / \mathrm{day}$, and after titration this reached $2000 \mathrm{mg} /$ day. $\chi^{2}$ analysis revealed only minor significant differences $(\mathrm{P}<$ 0.05 ) between LEV and TPM for comedication (Table 4).

Table 5 is an overview of the side effects of patients on LEV or TPM. The 22 patients who were on both drugs at $\mathrm{t}=0$ were eliminated from the analysis. 
Table 5 Side effects of patients on LEV and TPM ${ }^{\mathrm{a}}$.

\begin{tabular}{|c|c|c|c|c|c|c|}
\hline \multirow[t]{2}{*}{ Side effect } & \multicolumn{2}{|c|}{ 0-6 Months } & \multicolumn{2}{|c|}{ 6-18 Months } & \multicolumn{2}{|c|}{ 0-18 Months } \\
\hline & $\begin{array}{l}\text { LEV } \\
(n=247)\end{array}$ & $\begin{array}{l}\text { TPM } \\
(n=464)\end{array}$ & $\begin{array}{l}\text { LEV } \\
(n=197)\end{array}$ & $\begin{array}{l}\text { TPM } \\
(n=278)\end{array}$ & $\begin{array}{l}\text { LEV } \\
(n=247)\end{array}$ & $\begin{array}{l}\text { TPM } \\
(n=464)\end{array}$ \\
\hline Activating & $10^{* * *}$ & 36 & 10 & 9 & $15^{* * *}$ & 36 \\
\hline Deactivating & $13^{* * *}$ & 35 & $9^{* *}$ & 3 & $19^{* * *}$ & 36 \\
\hline Tiredness/sleepiness & $19^{* * *}$ & 48 & $9^{* *}$ & 17 & $23^{* * *}$ & 51 \\
\hline Total deactivating & $29^{* * *}$ & 50 & 16 & 18 & $36^{* * *}$ & 53 \\
\hline Headache & $3^{* * *}$ & 35 & 2 & 3 & $4^{* * *}$ & 35 \\
\hline Rest of CNS & $1^{* * *}$ & 34 & 2 & 3 & $2^{* * *}$ & 35 \\
\hline Food & 4 & 5 & $7^{* *}$ & 1 & $10^{*}$ & 6 \\
\hline
\end{tabular}

Table 6 Unadjusted (adjusted) odds ratios of occurrence of side effects in TPM as compared with LEV.

\begin{tabular}{lrlrlrl}
\hline & $0-6$ & Months & \multicolumn{3}{c}{$6-18$ Months } & \multicolumn{3}{c}{$0-18$ Months } \\
\hline Activating & 5.2 & $(7.6)$ & 0.8 & $(0.5)$ & 3.2 & $(4.7)$ \\
Deactivating & 3.7 & $(3.7)$ & 0.3 & $(0.3)$ & 2.4 & $(2.1)$ \\
Tiredness/sleepiness & 4.0 & $(3.4)$ & 2.0 & $(1.7)$ & 3.5 & $(3.4)$ \\
Total deactivating & 2.5 & $(2.2)$ & 1.1 & $(6.2)$ & 2.0 & $(1.8)$ \\
Headache & 20.0 & $(20.0)$ & 1.5 & $(2.5)$ & 14.0 & $(1.5)$ \\
Rest of CNS & 100.0 & $(33.3)$ & 1.5 & $(2.5)$ & 33.3 & $(20.0)$ \\
Food & 1.2 & $(1.5)$ & 0.1 & $(0.1)$ & 0.5 & $(0.7)$ \\
\hline
\end{tabular}

From 0 to 6 months a significantly higher percentage of TPM patients compared with LEV patients reported all categories of side effects except food side effects. At 0-6, $6-18$, and $0-18$ months, most of the patients who were tired/sleepy reported other deactivating side effects and vice versa, but there was much less of an overlap for LEV patients reporting tiredness/sleepiness and other deactivating side effects and vice versa. There was a decrease in all side effects for TPM and in reports of tiredness/ sleepiness for LEV after the first 6 months of treatment. Across time LEV appears to have equipotent activating and deactivating side effects. If we look at the overall picture, a large percentage of TPM patients reported activating and deactivating side effects, but for refractory patients we are interested in long-term drug administration. From 6 to 18 months TPM played a role predominantly as a sedative deactivator.

There is no significant difference between adjusted and unadjusted odds ratios for covariables of occurrence of side effects in TPM as compared with LEV patients for all side effects (Table 6). 


\section{I S C U S S I O N}

In the literature LEV is characterized as an activating $\mathrm{drug}^{8}$. In this study LEV was not found to have a pre-dominant activating or deactivating effect. In psychiatry, TPM is classified as a drug with deactivating and possibly antimanic effects ${ }^{8}$, but other depressive side effects in addition to tiredness/sleepiness have also been reported ${ }^{53,54}$. We found TPM to act as a deactivating AED with predominant tiredness/sleepiness side effects but not other deactivating side effects such as depression and anhedonia during long-term treatment. Compared with LEV, TPM was associated with a larger percentage of side effects and a larger percentage of withdrawals up to 6 months, but patients who got through the initial 6 months were likely to be suitable candidates for long-term treatment. Interestingly, during the first 6 months, there was a tendency for TPM patients to experience tiredness/sleepiness together with other deactivating effects but for LEV patients, tiredness/sleepiness reports were independent of other deactivating effects. The side effects of LEV were consistent over time, with the exception of tiredness/sleepiness, which occurred during initial titration of the drug. That these mood effects of TPM and LEV in this study were manifested at 18 months does not suggest an immediate drug effect that might be related to initial tolerance. This percentage is, however, not corrected for time on drug as some patients had started the drug recently; therefore, we overestimate the number of patients that will continue to take the drug and possibly underestimate the long-term side effects.

As the exact mode of action of TPM and LEV is incompletely understood, the mechanisms underlying the mood effects reported here remain unknown. Because the mechanism of action of LEV is still unclear, it is a difficult task to try and couple it to the hypothesis of Ketter et al. ${ }^{7}$. If we test this hypothesis the deactivating effects of TPM may be coupled to GABA potentiation. Both LEV and TPM attenuate glutamate-excitatory neurotransmission. Only TPM consistently enhances GABAinhibitory neurotransmission, and some authors have reported that it is unlikely that the action of LEV is mediated through the GABAergic system ${ }^{38,55}$. It is a possibility that there is a weak GABA link, but a simplistic bipolar hypothesis that does not take multiple mechanisms of action into account leads to confusing speculation.

The strengths of this study are the large sample number, the long follow-up evaluation, and the well-defined group of refractory patients. The shortcoming of this study is that it is a single-center unblinded, retrospective study. Clinician and researcher ratings were unblinded and might be biased by drug knowledge; however, it is very hard to follow refractory patients in a blinded fashion long term. This study was performed in a retrospective fashion, so any findings are limited by the design of the study. Due to the design of the study we cannot conclude how hazardous the activating and deactivating side effects were, but future studies should categorize 
both activating and deactivating side effects into desirable and undesirable effects in a $2 \times 2$ factorial design.

Unfortunately in the literature, the notes on behavior have changed over time and among reviewers who adjudicate the drug labeling information with varying specification of relevant details ${ }^{2}$. Because different coding dictionaries are used, different understandings of the same symptom may arise. Behaviors that might be coded with different terms include depression/apathy and aggression/hostility. The differences may be attributable to the way in which the sponsor presented information for review or to the way in which staff trained in epilepsy, but not in psychiatry, categorized $\operatorname{mood}^{56}$.

In this study, recording of behavior was subjective and not documented by formal objective rating scales. Formalized neuropsychological testing and demonstration of sustained behavioral effects would have added strength to the study. If coding were standardized the types of side effects related to AED use may be more consistent both within and between studies.

We feel that the data presented here are valid and that the large sample size is probably adequate to define typical experiences in patients with treatment-resistant epilepsy. However, inferring the precise incidence of adverse effects is hazardous and cannot substitute for further prospective investigations. Also, we must acknowledge that the side effects reported in this study may be attributable to several factors besides TPM or LEV administration. Care must be taken when differentiating between drug-related effects and the impact of the medical, psychiatric, and social aspects of epilepsy. This study would have been further validated if these variables had been investigated.

In this study we reviewed the literature on the mechanisms of action of TPM and LEV and attempted to couple these to the side effect profile of treatment-resistant epilepsy patients, paying particular attention to the Ketter et al. hypothesis? ${ }^{7}$ In the Ketter model, the distinction between mood-activating and mood-depressing effects of AEDs is a strong claim in our opinion, based on both experimental research and the clinical experience in both epilepsy and psychiatry; however, there is a much weaker link with the mechanisms of action.

We acknowledge that TPM and LEV have multiple mechanisms of action that are incompletely understood and reports of multiple side effects that we cannot conclusively relate to precise mechanisms of action. However, the hypothesis of Ketter et al. ${ }^{7}$ has enabled us to speculate that the difference in reported side effects may be partly attributable to differences in GABA involvement between TPM and LEV. Further research is needed to determine the attribution of both mood and GABA to neuroanatomic and neurochemical alterations in the brain both preclinically and clinically. This hypothesis may be useful but the association that is claimed in the Ketter model of a direct link between the mechanisms of action of the AEDs and mood effects is 
no more than a hypothetical claim, based partly on a simplified model of the GABA versus glutamate mechanisms of AEDs without the consideration of other mechanisms of AEDs that could be relevant. Additionally, it does not take into account the precise type of GABA/glutamate modulation. It is likely that more detailed coupling of the mechanisms of action and side effects of TPM and LEV and an elaboration of the hypothesis of Ketter et al. ${ }^{7}$ may give less confusing speculations. Understanding the mechanisms of action of anticonvulsants integrated with clinical and experimental investigations may ultimately provide new insights into the pathophysiology and treatment of epilepsy and psychological outcome measures. 


\section{R E FER E NCES}

1. White S. Clinical significance of animal seizure models and mechanism of action studies of potential antiepileptic drugs. Epilepsia 1997;38:9-17.

2. Cramer JA, Ben Menachem E, French J. Review of treatment options for refractory epilepsy: new medications and vagal nerve stimulation. Epilepsy Res 2001;47:17-25.

3. Bootsma HP, Coolen F, Aldenkamp AP, et al. Topiramate in clinical practice: long-term experience in patients with refractory epilepsy referred to a tertiary epilepsy center. Epilepsy Behav 2004;5:380-7.

4. Cramer JA, Leppik IE, Rue KD, Edrich P, Kramer G. Tolerability of levetiracetam in elderly patients with CNS disorders. Epilepsy Res 2003;56:135-45.

5. Torta R, Keller R. Behavioral, psychotic, and anxiety disorders in epilepsy: etiology, clinical features, and therapeutic implications. Epilepsia 1999;40(Suppl. 10):S12-20.

6. Ben-Menachem E, Gilland E. Efficacy and tolerability of levetiracetam during 1-year follow-up in patients with refractory epilepsy. Seizure 2003;12:131-5.

7. Ketter TA, Post RM, Theodore WH. Positive and negative psychiatric effects of antiepileptic drugs in patients with seizure disorders. Neurology 1999;53(5, Suppl. 2):S53-67.

8. Reijs R, Aldenkamp AP, De Krom M. Mood effects of antiepileptic drugs. Epilepsy Behav 2004;5(Suppl.1):S66-76.

9. Korean Topiramate Study Group. Topiramate in medically intractable partial epilepsies: double-blind placebo-controlled randomized parallel group trial. Epilepsia 1999;40:1767-74.

10. Ben-Menachem E, Henriksen O, Dam M, et al. Double-blind, placebo-controlled trial of topiramate as add-on therapy in patients with refractory partial seizures. Epilepsia 1996;37:539-43.

11. Faught E, Wilder BJ, Ramsay RE, et al. for the Topiramate YD Study Group. Topiramate placebocontrolled dose-ranging trial in refractory partial epilepsy using 200-, 400-, and 600-mg daily dosages. Neurology 1996;46:1684-90.

12. Privitera M, Fincham R, Penry J, et al. for the Topiramate YE Study Group. Topiramate placebocontrolled, dose-ranging trial in refractory partial epilepsy using 600-, 800-, and 1,000-mg daily dosages. Neurology 1996;46:1678-83.

13. Sharief M, Viteri C, Ben-Menachem E, et al. Double-blind, placebo-controlled study of topiramate in patients with refractory partial epilepsy. Epilepsy Res 1996;25:217-24.

14. Tassinari CA, Michelucci R, Chauvel P, et al. Double-blind, placebo-controlled trial of topiramate (600 mg daily) for the treatment of refractory partial epilepsy. Epilepsia 1996;37:763-8.

15. Yen DJ, Yu HY, Guo YC, Chen C, Yiu CH, Su MS. A double blind, placebo-controlled study of topiramate in adult patients with refractory partial epilepsy. Epilepsia 2000;41:1162-6.

16. Wang Y, Zhou D, Pauli E, Stefan H. Topiramate on ictal seizure semiology: a quantitative, randomized, low and medium dose controlled study. Epilepsy Res 2001;46:271-7.

17. Cereghino JJ, Biton V, Abou-Khalil B, Dreifuss F, Gauer LJ, Leppik I. Levetiracetam for partial seizures: results of a double blind, randomized clinical trial. Neurology 2000;55:236-42.

18. Shorvon SD, Lowenthal A, Janz D, Bielen E, Loiseau P, for the European Levetiracetam Study Group. Multicenter double-blind, randomized, placebo-controlled trial of levetiracetam as add-on therapy in patients with refractory partial seizures. Epilepsia 2000;41:1179-86. 
19. Ben-Menachem E, Falter U, for the European Levetiracetam Study Group. Efficacy and tolerability of levetiracetam $3000 \mathrm{mg} / \mathrm{d}$ in patients with refractory partial seizures: a multicenter, double blind, responder-selected study evaluating monotherapy. Epilepsia 2000;41:1276-83.

20. McElroy SL, Suppes T, Keck PE, et al. Open-label adjunctive topiramate in the treatment of bipolar disorders. Biol Psychiatry 2000;47:1025-33.

21. Calabrese JR, Keck Jr PE, McElroy SL, Shelton MD. A pilot study of topiramate as monotherapy in the treatment of acute mania. J Clin Psychopharmacol 2001;21:340-2.

22. Calabrese JR, Shelton MD, Rapport DJ, Kimmel SE. Bipolar disorders and the effectiveness of novel anticonvulsants. J Clin Psychiatry 2002;63(Suppl. 3):5-9.

23. Carpenter LL, Leon Z, Yasmin S, Price LH. Do obese depressed patients respond to topiramate? A retrospective chart review. J Affect Disord 2002;69:251-5.

24. Shapira NA, Goldsmith TD, McElroy SL. Treatment of binge eating disorder with topiramate: a clinical case series. J Clin Psychiatry 2000;61:368-72.

25. Shank RP, Gardocki JF, Vaught JL, et al. Topiramate: preclinical evaluation of structurally novel anticonvulsant. Epilepsia 1994;35:450-60.

26. Klitgaard H, Matagne A, Gobert J, Wulfert E. Evidence for a unique profile of levetiracetam in rodent models of seizures and epilepsy. Eur J Pharmacol 1998;353:191-206.

27. Loscher W, Honack D. Profile of ucb L059, a novel anticonvulsant drug, in models of partial and generalized epilepsy in mice and rats. Eur J Pharmacol 1993;232:147-58.

28. Vaught JL, Maryanoff BE, Shank RP. The pharmacological profile of topiramate: a structurally novel, clinically and effective anticonvulsant. Epilepsia 1991;32(3).

29. Gower AJ, Noyer M, Verloes R, Gobert J, Wulfert E. ucb L059, a novel anti-convulsant drug: pharmacological profile in animals. Eur J Pharmacol 1992;222:193-203.

30. Nakamura J, Tamura S, Kanda T, et al. Inhibition by topiramate of seizures in spontaneously epileptic rats and DBA/2 mice. Eur J Pharmacol 1994;254:83-9.

31. Gower AJ, Hirsch E, Boehrer A, Noyer M, Marescaux C. Effects of levetiracetam, a novel antiepileptic drug, on convulsant activity in two genetic rat models of epilepsy. Epilepsy Res 1995;22:207-13.

32. Amano K, Hamada K, Yagi K, Seino M. Antiepileptic effects of topiramate on amygdaloid kindling in rats. Epilepsy Res 1998;31:123-8.

33. Wauquier A, Zhou S. Topiramate: a potent anticonvulsant in the amygdala-kindled rat. Epilepsy Res 1996;24:73-7.

34. Simeone TA, McClellan AML, Trwyman RE, White HS. Direct activation of the recombinant a413s GABAa receptor by the novel anticonvulsant topiramate. Soc Neurosci Abstr 2000;26:237.

35. White HS, Brown D, Skeen GA. The anticonvulsant topiramate displays a unique ability to potentiate GABA-evoked chloride currents. Epilepsia 1995;36:39-40.

36. Petroff OA, Hyder F, Mattson RH, Rothman DL. Topiramate increases brain GABA, homocarnosine, and pyrrolidinone in patients with epilepsy. Neurology 1999;52:473-8.

37. Herrero AI, Del Olmo N, Gonzalez-Escalada JR, Solis JM. Two new actions of topiramate: inhibition of depolarizing GABA (A)-mediated responses and activation of a potassium conductance. Neuropharmacology 2002;42:210-20. 
38. Rigo JM, Nguyen L, Rocher V. Levetiracetam: novel modulation of ionotropic inhibitory receptors. Epilepsia 2000;41(35).

39. Poulain P, Margineanu DG. Levetiracetam opposes the action of GABAA antagonists in hypothalamic neurones. Neuropharmacology 2002;42:346-52.

40. Loscher W, Honack D, Bloms-Funke P. The novel antiepileptic drug levetiracetam (ucb L059) induces alterations in GABA metabolism and turnover in discrete areas of rat brain and reduces neuronal activity in substantia nigra pars reticulata. Brain Res 1996;735:208-16.

41. Gibbs 3rd JW, Sombati S, DeLorenzo RJ, Coulter DA. Cellular actions of topiramate: blockade of kainate-evoked inward currents in cultured hippocampal neurons. Epilepsia 2000;41(Suppl. 1):S10-6.

42. Skradski S, White HS. Topiramate blocks kainate-evoked cobalt influx into cultured neurons. Epilepsia 2000;41(Suppl. 1):S45-7.

43. Hans G, Rigo JM, Crommen J. Levetiracetam: no relevant effect on ionotropic excitatory glutamate receptors. Epilepsia 2000;41(37).

44. Coulter DA, Sombati S, DeLorenzo RJ. Selective effects of topiramate on sustained repetitive firing and spontaneous bursting in cultured hippocampal neurons. Epilepsia 1993;34:122.

45. Sombati S, Coulter DA, DeLorenzo RI. Effects of topiramate on sustained reletitive firing and low Mg2+-induced seizure discharges in cultured hippocampal neurons. Epilepsia 1995;36:38.

46. Zona C, Marchetti C, Margineanu DG. The novel antiepileptic drug candidate levetiracetam does not modify the biophysical properties of the $\mathrm{Na}+$ channel in rat cortical neurons in culture. Soc Neurosci Abstr 1999;25:1868.

47. Qian J, Noebels JL. Topiramate alters excitatory synaptic transmission in mouse hippocampus. Epilepsy Res 2003;55:225-33.

48. Zhang X, Velumian AA, Jones OT, Carlen PL. Modulation of high-voltage-activated calcium channels in dentate granule cells by topiramate. Epilepsia 2000;41(Suppl. 1):S52-60.

49. Zona C, Niespodziany I, Marchetti C, Klitgaard H, Bernardi G, Margineanu DG. Levetiracetam does not modulate neuronal voltage-gated $\mathrm{Na}+$ and T-type Ca2+ currents. Seizure 2001;10:279-86.

50. Margineanu DG, Wulfert E. Inhibition by levetiracetam of a non- GABAA receptor-associated epileptiform effect of bicuculline in rat hippocampus. Br J Pharmacol 1997;122:1146-50.

51. Margineanu DG, Wulfert E. ucb L059, a novel anticonvulsant, reduces bicuculline-induced hyperexcitability in rat hippocampal CA3 in vivo. Eur J Pharmacol 1995;286:321-5.

52. Madeja M, Margineanu DG, Klitgaard H. Effects of levetiracetam on voltage-gated potassium channels: a novel antiepileptic mechanism of action?. Epilepsia 2001;42:19.

53. Tatum WOT, French JA, Faught E, et al. Postmarketing experience with topiramate and cognition. Epilepsia 2001;42:1134-40.

54. Shorvon SD. Safety of topiramate: adverse events and relationships to dosing. Epilepsia 1996;37(Suppl. 2):S18-22.

55. Sills GJ, Leach JP, Fraser CM, Forrest G, Patsalos PN, Brodie MJ. Neurochemical studies with the novel anticonvulsant levetiracetam in mouse brain. Eur J Pharmacol 1997;325:35-40.

56. Levinson DF, Devinsky O. Psychiatric adverse events during vigabatrin therapy. Neurology 1999;53:1503-11. 


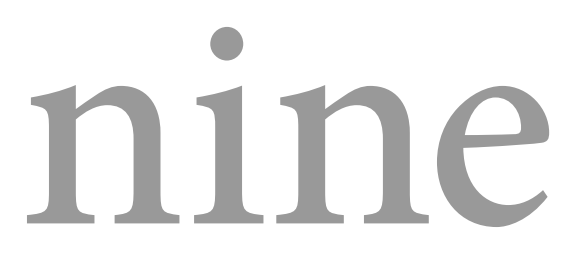

\section{The impact of side effects on long-term retention in three new anti-epileptic drugs}

H.P.R. Bootsma, L. Ricker, Y.A. Hekster, J. Hulsman, D. Lambrechts, M. Majoie, A. Schellekens, M.C.T.F.M. de Krom, A.P. Aldenkamp Accepted for publication in Seizure 
Objective: To determine long-term retention, percentage of patients withdrawing because of adverse events, percentage of patients achieving seizure freedom, safety profile of the new anti-epileptic drugs lamotrigine, levetiracetam and topiramate.

Methods: all patients treated with lamotrigine, levetiracetam or topiramate in the Epilepsy Centre were identified. Each drug was analyzed from introduction of the drug in the Netherlands up to a final assessment point 2 years later.

Results: data from 1066 patients were included: 336 for lamotrigine, 301 for levetiracetam, 429 for topiramate. Two-year retention rates were 69.2\% (lamotrigine), $45.8 \%$ (levetiracetam), 38.3\% (topiramate); (LTG vs. LEV at $\mathrm{p}<0.001$; LTG vs. TPM at $\mathrm{p}<0.001 ; \mathrm{LEV}$ vs. TPM at $\mathrm{p}=0.005)$. Seizure freedom rates were lowest for lamotrigine and highest for levetiracetam. Adverse events played a role in drug discontinuation in $154 / 429$ patients $(35.9 \%)$ on topiramate, $52 / 336$ patients $(15.5 \%)$ on lamotrigine $(\mathrm{p}<0.001), 68 / 301$ patients $(22.5 \%)$ on levetiracetam $(\mathrm{p}<0.001)$. Mood and general CNS-effects are common in patients on lamotrigine and levetiracetam, and neurocognitive side effects are most prevalent in patients on topiramate. A positive effect on cognition is frequently noted in patients on lamotrigine.

Conclusion: a drug that is only modestly efficacious but has a favourable safety profile may look better than a drug that is more efficacious but produces clinically meaningful adverse events. Therefore, a drug's retention rate is mainly determined by its side effect profile. As a consequence, retention rate was highest for lamotrigine and lowest for topiramate. Intermediate retention rates were seen with levetiracetam use. 


\section{N T RODUCT IO N}

The primary goals of antiepileptic drug (AED) treatment are to achieve complete seizure freedom, ideally without adverse events, reduce morbidity, mortality, and seizure related accidents, and improve quality of life (Sander, 2004). In two-thirds of the patients with epilepsy these goals are feasible with optimum AED therapy. For the remaining one-third of patients with refractory epilepsies these goals are not reached with existing AEDs and, the introduction of several new AEDs in the recent past might be a welcome development for improved patient treatment. Some studies (Luciano \& Shorvon, 2007; Callaghan et al., 2007) have shown results of treatment changes in an apparent refractory population.

With a growing number of AEDs, it becomes more difficult for clinicians to make a rational choice which drug to prescribe for which patient. To date, treatment of epilepsy is rather more empirical than evidence-based (Sander, 2004). In an ideal situation, treatment guidelines will use information from large multicenter randomized controlled trials that compare AEDs in a head to head fashion. These trials are however rarely performed. In the absence of such trials, other methods must be used to compare new AEDs on their intrinsic properties. The relevance of findings derived from meta-analyses to clinical practice is limited. Studies included in meta-analyses are typically of short duration among other limitations (Otoul et al., 2005), whereas epilepsy is a chronic condition. Therefore, long-term open label observational studies and clinical practice audits better reflect everyday clinical practice regarding longterm efficacy and safety issues (Mohanraj and Brodie, 2003).

One way to compare the long-term performances of AEDs in clinical practice is to evaluate retention rates. Prolonged retention of patients on their AED therapy is now accepted as one of the clearest reflections of therapeutic efficiency and represents a clinically meaningful composite measure of both efficacy and safety over time (Lhatoo et al., 2000). Retention-time studies are especially useful when the actual seizure-frequency reduction per patient and the percentage of seizure-free patients are determined (Knoester et al. 2005). Presently, it is not clear whether efficacy and safety contribute equally to the retention rate of new AEDs. One study mentions that retention rate may reflect more of AED tolerability rather than efficacy (Chung et al., 2007).

This audit focuses on the three most commonly prescribed new AEDs in patients with refractory epilepsies in a tertiary referral center for epilepsy: lamotrigine (LTG), levetiracetam (LEV) and topiramate (TPM). Our objectives were to compare the longterm retention, the percentage of patients withdrawing because of adverse events, the percentage of patients achieving seizure freedom, and the safety profile of LTG, LEV and TPM. 
The study was approved by the institutional Medical Ethics Committee. All in- and out-patients who had been treated with LTG, LEV or TPM in a certain period in the Epilepsy Centre Kempenhaeghe were identified by means of our automated medical information system (MIS) and subsequently analyzed. Each drug was analyzed from the introduction of the drug in the Netherlands up to a final assessment point (TPM 1993-2002; LTG 1996-1997; LEV 2001-2003). Patients who had the drug of interest prescribed initially elsewhere were not included to avoid survival bias. Retention times were calculated from the time the patients started to take the drug of interest to the time they discontinued treatment, with a maximum follow-up period of 24 months. Data from patients who were still taking the drug at the time of data collection were counted as censored data.

A standardized data form was developed. The data were obtained from our Medical Information System and individual patient medical records. Epilepsy and seizures were classified using the International League Against Epilepsy (ILAE) classification. Treatment was evaluated every 6 months for each drug. Mood disorders due to the use of AEDs were classified into activating and sedating effects, with the former leading to aggression, hyperirritability, and agitation, and the latter leading to apathy and depression (Ketter et al., 1999, Roberts et al., 2005). Data forms were entered into a computerized database for analysis. Statistical analysis was performed using SPSS 15.0 for Windows. Retention rates were calculated by using Kaplan-Meier survival analysis (Kaplan, 1958), and comparisons between the retention curves were analyzed using log-rank tests. Other comparisons between the drugs were analyzed using non-parametric statistics, Pearson $\chi^{2}$.

\section{R E S U L T S}

One hundred of 1166 patients had the drug of interest prescribed initially elsewhere or data were lacking. Therefore, data from 1066 patients were included in the analysis: 429 for TPM, 336 for LTG, and 301 for LEV. The main characteristics of patients are given in table 1. Populations representing LTG, LEV, and TPM were similar for the most important demographic and clinical variables and represent the typical group of refractory patients referred to a tertiary epilepsy centre.

Kaplan-Meier survival analysis, shown by figure 1, revealed significant differences between the drugs. Estimated retention rates of 75.2\% (LTG), 65.6\% (LEV), and 51.7\% (TPM) were reported at one year (LTG vs. LEV at $\mathrm{p}=0.008$; LTG vs. TPM at $\mathrm{p}<0.001$; LEV vs. TPM at $p=0.002$ ). Retention at two years had decreased to $69.2 \%$ for LTG, 
45.8\% for LEV, and 38.3\% for TPM (LTG vs. LEV at $\mathrm{p}<0.001$; LTG vs. TPM at $\mathrm{p}<$ $0.001 ;$ LEV vs. TPM at $\mathrm{p}=0.005$ ).

Figure 2 shows the percentages of patients that are free of seizures from baseline. Seizure freedom rates were lowest for LTG and highest for LEV at all assessment points. No statistically significant differences between the seizure freedom rates could be identified.

Adverse events played a role in drug discontinuation in 154 of 429 patients (35.9\%) on TPM. Significantly less patients discontinued drug treatment because of adverse events in the other populations: 52 of 336 patients $(15.5 \%)$ on LTG $(\mathrm{p}<0.001)$ and 68 of 301 patients $(22.5 \%)$ on LEV ( $p<0.001$ ). The difference between LTG and LEV is also significant $(\mathrm{p}=0.028)$.

The most frequently reported side effects in patients who discontinued treatment are listed in table 2. Effects on mood and general CNS-effects (dizziness, tiredness, sleepiness) were common side effects at time of discontinuation in patients on LTG, LEV, and TPM. In patients on TPM, the neurocognitive side effects like mental slowing and dysphasia were most important and contributed to almost half of patients in discontinuing treatment. Rash played a role in drug discontinuation in both the LTG and TPM study populations.

The most frequently reported side effects per assessment point are shown in table 3. Similarly, effects on mood and general CNS-effects are common side effects in patients on LTG and LEV, and neurocognitive side effects are most prevalent in patients on TPM. A positive effect on behaviour is most frequently noted in patients on LTG. Improved alertness, emotional stability, and reduced irritability were frequently noted. This effect is seen in a substantial number of patients on LEV only in the first six months, and not at all in patients on TPM.

\section{I S C USS I O N}

This audit compared the use of LTG, LEV, and TPM, presently three of the most commonly prescribed new AEDs in chronic epilepsy. The study population consisted of patients with chronic refractory epilepsy referred to a single tertiary epilepsy centre. The added value of this study is due to the direct comparison of the longterm performance of LTG, LEV, and TPM including seizure freedom rates and safety issues, the large number of patients included ( $\mathrm{n}=336$ for LTG; $\mathrm{n}=301$ for LEV; $\mathrm{n}=$ 429 for TPM), the long period of evaluation (24 months), and the low risk of selection bias by the inclusion of all patients who were started on LTG, LEV, or TPM in a certain period. This is illustrated by the characteristics of our study population, specifically by a wide age range and a large number of children (22\%) and mentally retarded (33\%). The patients on each drug did not differ on any of the clinical or 
demographical variables, which allows us to compare LTG, LEV, and TPM and to assume that differences in outcome are drug-related.

Many studies have been done on individual new AEDs as add-on treatment. However, few compare these new AEDs with each other. Ideally, neurologists make treatment decisions based on results from long-term randomized controlled trials that make head-to-head comparisons. These trials are rarely performed because of specific reasons (Privitera, 2006). Therefore, results from long-term observational studies become increasingly more important, since these studies give clinicians insight in the long-term performance of new AEDs in terms of retention rate, seizure control, and safety profile.

One way to compare the long-term performance of new AEDs in observational studies is to evaluate retention rates. Retention rate is considered to be a composite measure of drug efficacy and drug safety and expresses the willingness of patients to continue drug treatment. Therefore, it is clinically the most relevant parameter of an AED. In our study, retention rate at two years was highest with LTG (69.2\%), followed by LEV (45.8\%), and TPM (38.3\%). Chung et al showed similar two-year retention rates of $74.1 \%$ for LTG, $53.6 \%$ for LEV, and $44.2 \%$ for TPM (Chung et al., 2007). In addition, a study executed in a residential community of adults with chronic epilepsy and learning disability found a higher retention rate at 5 years in patients on LTG (52\%) compared to patients on LEV (32\%) and TPM (28\%). However, two-year retention rates for LTG and LEV were similar (57\% and 56\%, respectively) (Simister et al., 2007).

Contrary to those studies and most other published follow-up studies, we obtained estimates of the proportion of patients with complete seizure control for at least 6 months. This is relevant, since these figures provide clinically the most meaningful data for predicting the long-term efficacy of an AED (Chadwick, 1998). This is especially important since in this study the category of patients were regarded as drug resistant and the new AEDs have substantially added value in this population. Surprisingly, higher seizure freedom rates were not associated with higher retention rates. Retention rate for LTG was highest whereas seizure freedom rates, although not statistically significant, were lowest. Long-term retention for TPM was dramatically lower than for LTG, whereas seizure freedom rates were higher. This suggests that the impact of the achievement of seizure remission on long-term retention is considered less important. Moreover, it should be noted that not all patients who continue on therapy do so because of improvement in seizure frequency. Some patients continued drug treatment while experiencing even a higher seizure frequency.

As a marker of tolerability we used the percentage of patients withdrawing drug treatment because of adverse events. TPM was by far the least well-tolerated drug, followed by LEV, and LTG. AED-specific adverse events that often led to discontinuation of drug treatment were mental slowing and dysphasia in patients on TPM, mood 
Table 1 Patient demographics and characteristics.

\begin{tabular}{|c|c|c|c|}
\hline & LTG & LEV & TPM \\
\hline Total no. of patients & 336 & 301 & 429 \\
\hline Age & $32.8 \pm 16.4$ & $32.5 \pm 16.8$ & $34.9 \pm 8.6$ \\
\hline Age range & $1-74$ & $1-75$ & $1-73$ \\
\hline Age distribution & $20.8 \%<18$ & $19.9 \%<18$ & $24.5 \%<18$ \\
\hline $\operatorname{Sex}(F / M)$ & $170 / 166$ & $156 / 145$ & $204 / 225$ \\
\hline Weight (kg) & $63.3 \pm 20.6$ & $50.5 \pm 21.6$ & $54.0 \pm 26.6$ \\
\hline \multicolumn{4}{|l|}{ Type of epilepsy } \\
\hline Localization related & $231(68.8 \%)$ & $217(72.1 \%)$ & $323(75.3 \%)$ \\
\hline Generalized & $63(18.8 \%)$ & $54(17.9 \%)$ & $86(20.1 \%)$ \\
\hline Undetermined & $8(2.4 \%)$ & $16(5.4 \%)$ & $10(2.3 \%)$ \\
\hline Not classified & $34(10.1 \%)$ & $14(4.6 \%)$ & $10(2.3 \%)$ \\
\hline \multicolumn{4}{|l|}{ Seizure type } \\
\hline Partial onset & $272(81.0 \%)$ & $243(80.7 \%)$ & $352(82.1 \%)$ \\
\hline Generalized & $182(54.2 \%)$ & $138(45.8 \%)$ & $209(48.7 \%)$ \\
\hline Not classified & $21(6.3 \%)$ & $19(6.3 \%)$ & $19(4.4 \%)$ \\
\hline Nonepileptic attacks & $36(10.7 \%)$ & $28(9.3 \%)$ & $31(7.2 \%)$ \\
\hline Age at onset of seizures & $11.0 \pm 11.2$ & $11.7 \pm 11.7$ & $9.7 \pm 11.1$ \\
\hline $\begin{array}{l}\text { Duration of epilepsy before } \\
\text { drug introduction (years) }\end{array}$ & $22.0 \pm 14.1$ & $21.3 \pm 12.9$ & $21.1 \pm 13.4$ \\
\hline No. mentally retarded & $102(30.4 \%)$ & $98(32.6 \%)$ & $158(36.8 \%)$ \\
\hline CT abnormalities & $19.0 \%$ & $17.3 \%$ & $18.9 \%$ \\
\hline MRI abnormalities & $25.0 \%$ & $31.9 \%$ & $36.4 \%$ \\
\hline No. of concomitant AEDs & $2.09 \pm 0.847$ & $2.16 \pm 0.827$ & $2.04 \pm 0.779$ \\
\hline \multicolumn{4}{|c|}{ Most frequently used concomitant AEDs at baseline } \\
\hline carbamazepine & $53.0 \%$ & $39.9 \%$ & $44.8 \%$ \\
\hline clobazam & $38.1 \%$ & $37.6 \%$ & $34.3 \%$ \\
\hline phenytoin & $24.4 \%$ & $15.6 \%$ & $19.3 \%$ \\
\hline oxcarbazepine & $16.7 \%$ & $16.3 \%$ & $14.5 \%$ \\
\hline valproic acid & $21.4 \%$ & $19.3 \%$ & $18.4 \%$ \\
\hline
\end{tabular}

disorders in patients on LEV, and rash in patients on LTG, as previously reported in other studies (Chung et al., 2007, Simister et al., 2007, Tatum et al., 2001, Weintraub et al.,2007, White et al., 2003, Blum et al., 2006). As expected, we found a correlation between side effects and long-term retention: the higher the percentages of patients withdrawing because of adverse events, the lower the retention rate. 


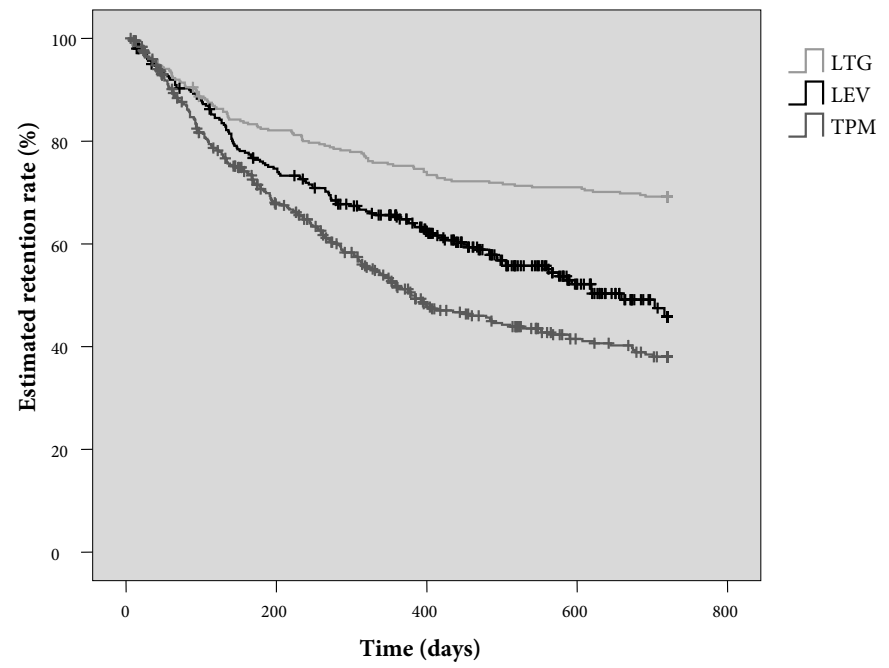

Figure 1 Estimated retention rates by Kaplan-Meier survival analysis; comparison between LTG, LEV, and TPM.

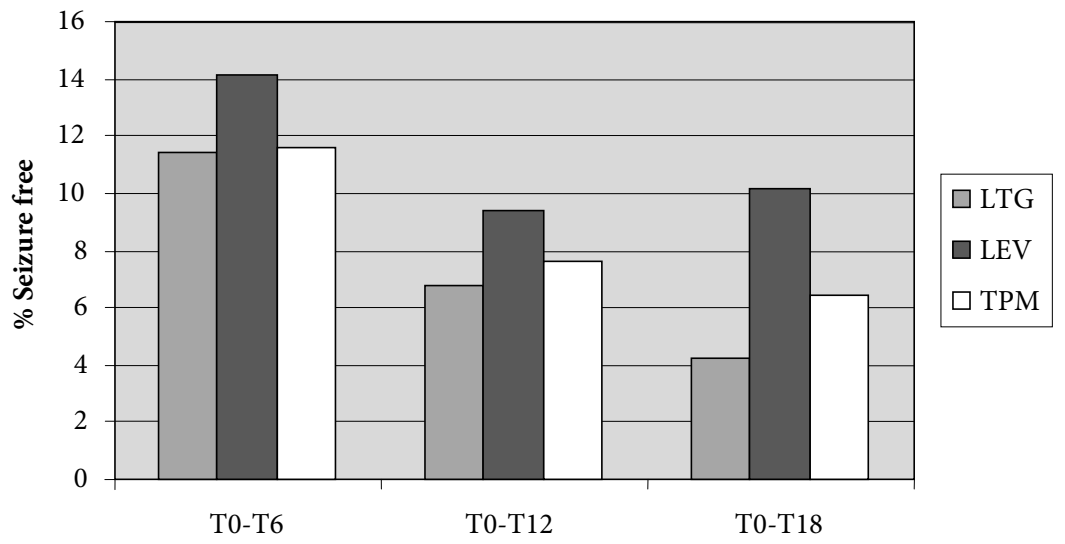

Figure 2 Long-term seizure remission from baseline; comparison between LTG, LEV, and TPM.

Several findings with respect to retention rates were noteworthy in our study. Firstly, retention rates could have been influenced by the sequence in which LTG, LEV, and TPM were marketed. Patients on drugs that were marketed first could have been withdrawn from treatment because of the availability of a new AED. On the other 


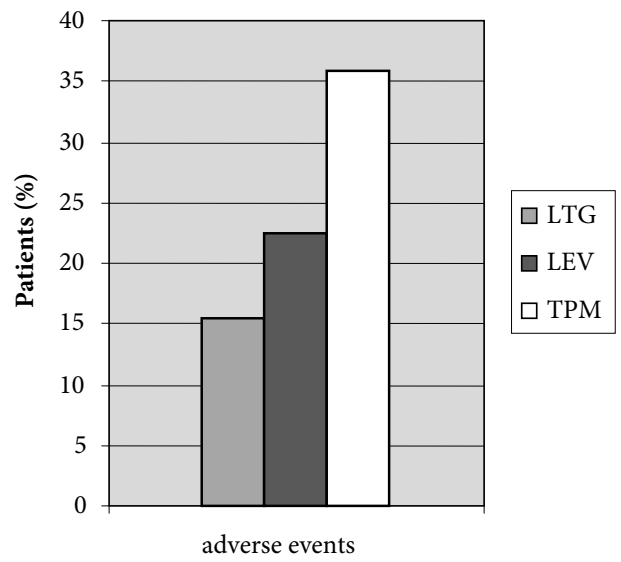

Figure 3 Percentage of total study population in which adverse events played a role in drug discontinuation; comparison between LTG, LEV, and TPM.

hand, drugs that were marketed later could have been tested in a more refractory population.

Secondly, if patients did stop taking the audit drug, they were most likely to do this in the first 6 to 2 months, mostly due to adverse events. This implicates that surviving the toxic effects of an AED in the early stage is a good indicator of long-term retention. This is also mentioned by other studies reporting continuation rates of about 90\% for LTG, LEV, and TPM after surviving the initial stage of AED introduction (Chung et al., 2007, Chappell and Crawford, 2005).

Thirdly, cognitive impairment is a common side effect in patients using AEDs (Brunbach and Sabers, 2002). Across studies, LTG adjunctive therapy did not worsen, and sometimes improved, preexisting cognitive dysfunction in patients with epilepsy (Aldenkamp and Baker, 2001). In one study, only 11 of 81 patients (13.6\%) achieved a $50 \%$ reduction in seizure frequency on LTG, whereas more than $50 \%$ chose to continue LTG therapy, indicating that other factors influenced their decision (Smith et al., 1993). In contrast, TPM has been associated with cognitive impairment, particularly in verbal function, memory, and attention in patients with epilepsy (Tatum et al., 2001, Aldenkamp et al., 2000, Fritz et al., 2005). In our study, almost half of patients on TPM discontinued treatment because of the drug's negative effects on cognition. However, patients on LTG frequently experienced a positive effect on cognition. Patients were less irritable, more alert, and emotionally stable. We think that the side effect profile, and especially the impact of an AED on cognitive function, is the main determinant of retention rate. Therefore, effects on cognition constitute a key consideration in selecting antiepileptic drugs. 
Table 2 Reported side effects in patients who discontinued drug treatment ${ }^{\mathrm{a}}$.

\begin{tabular}{lll}
\hline LTG & LEV & TPM \\
\hline Dizziness (14.9\%) & Mood disorders $\uparrow^{\mathrm{b}}(13.8 \%)$ & Mental slowing (27.8\%) \\
Mood disorders $\uparrow(11.7 \%)$ & Tiredness (13.8\%) & Dysphasia $^{\mathrm{c}}(15.0 \%)$ \\
Rash (10.6\%) & Mood disorders $\downarrow^{\mathrm{d}}(13.1 \%)$ & Mood disorders $\uparrow(13.2 \%)$ \\
Sleeplessness (7.4\%) & Sleepiness (8.5\%) & Gastrointestinal (10.6\%) \\
Sleepiness (6.4\%) & & Paresthesia (7.5\%) \\
& & Appetite loss (7.0\%) \\
& Skin (6.6\%) \\
& Weight loss (6.2\%) \\
& Mood disorders $\downarrow$ (5.7\%) \\
& & Headache (5.7\%) \\
& & Dizziness (5.3\%) \\
\hline
\end{tabular}

a Only side effects that occurred in $>5 \%$ of patients are reported in the table; ${ }^{\mathrm{b}}$ Mood disorders $\uparrow:$ agitation, aggression, hyperirritability; ${ }^{\mathrm{c}}$ Dysphasia: word-finding difficulties; ${ }^{\mathrm{d}}$ Mood disorders $\downarrow$ : depression, apathy.

Table 3 Most frequently reported side effectsa.

\begin{tabular}{|c|c|c|c|}
\hline & LTG & LEV & TPM \\
\hline \multirow[t]{3}{*}{6 months } & Positive behaviour ${ }^{\mathrm{b}}(17.2 \%)$ & Mood disorders $\uparrow^{c}(8.1 \%)$ & Mental slowing (13.8\%) \\
\hline & Dizziness (11.2\%) & Tiredness $(7.4 \%)$ & Dysphasia $^{\mathrm{d}}(6.6 \%)$ \\
\hline & Gastrointestinal (5.7\%) & Positive behaviour (7.4\%) & Weight loss (5.2\%) \\
\hline \multirow[t]{2}{*}{12 months } & Positive behaviour (9.1\%) & Mood disorders $\uparrow(5.2 \%)$ & Mental slowing (13.6\%) \\
\hline & Dizziness $(6.9 \%)$ & & Dysphasia (5.7\%) \\
\hline \multirow[t]{3}{*}{18 months } & Positive behaviour (8.8\%) & Mood disorders $\uparrow(10.6 \%)$ & Mental slowing (8.1\%) \\
\hline & Dizziness $(8.8 \%)$ & Tiredness $(6.4 \%)$ & Dysphasia (6.5\%) \\
\hline & & Mood disorders $\uparrow(5.7 \%)$ & \\
\hline \multirow[t]{2}{*}{24 months } & Positive behaviour (7.2\%) & $\begin{array}{l}\text { (Number of patients too } \\
\text { small to provide meaningful } \\
\text { data) }\end{array}$ & Urogenitale $^{\mathrm{e}}(5.6 \%)$ \\
\hline & Dizziness (5.1\%) & & \\
\hline
\end{tabular}

a Only side effects that occurred in $>5 \%$ of the patients are reported in the table; ${ }^{\text {b }}$ Positive behaviour: improved alertness, emotional stability, reduced irritability, etc.; ${ }^{c}$ Mood disorders $\uparrow$ : agitation, aggression, hyperirritability; ${ }^{\mathrm{d}}$ Dysphasia: word-finding difficulties; ${ }^{\mathrm{e}}$ Urogenital complaints: impotence, amenorrhea, micturation problems, etc.

To conclude: the new AEDs have substantially added value in the group of drug resistant epilepsy patients. In addition a drug that is only modestly efficacious but has a favourable safety profile may seem better than a drug that is more efficacious but produces clinically meaningful adverse events. Therefore, a drug's retention rate is mainly determined by its side effect profile. The gain in quality of life after drug initiation determines the continuation rate of an individual AED, with the effects on 
cognition being far more important than seizure control. As a consequence, retention rate was highest for LTG and lowest for TPM. Intermediate retention rates were seen with LEV use. 


\section{R E FER E NCES}

Aldenkamp AP, Baker G, Mulder OG, et al (2000) A multicenter, randomized clinical study to evaluate the effect on cognitive function of topiramate compared with valproate as add-on therapy to carbamazepine in subjects with partial-onset seizures. Epilepsia 41:1167-78.

Aldenkamp AP, Baker G (2001) A systematic review of the effects of lamotrigine on cognitive function and quality of life. Epilepsy Behav 2:85-91.

Blum D, Meador K, Biton V, et al (2006) Cognitive effects of lamotrigine compared with topiramate in patients with epilepsy. Neurology 67:400-6.

Brunbech L, Sabers A (2002) Effect of antiepileptic drugs on cognitive function in individuals with epilepsy. Drugs 62:593-604.

Callaghan BC (2007) Likelihood of seizure remission in an adult population with refractory epilepsy. Ann Neurol 62:382-389

Chadwick DW. Report of the ILAE commission on antiepileptic drugs. Considerations on designing clinical trials to evaluate the place of new antiepileptic drugs in the treatment of newly diagnosed and chronic patients with epilepsy. Epilepsia 39:799-803.

Chappell B, Crawford P (2005) An audit of lamotrigine, levetiracetam and topiramate usage for epilepsy in a district general hospital. Seizure 14:422-8.

Chung S, Wang N, Hank N (2007) Comparative retention rates and long-term tolerability of new antiepileptic drugs. Seizure16:296-304.

Fritz N, Glogau S, Hoffmann J, Rademacher M, Elger CE, Helmsteadter C (2005) Efficacy and cognitive side effects of tiagabine and topiramate in patients with epilepsy. Epilepsy Behav 6:373-81.

Kaplan EL (1958) Non-parametric estimation from incomplete observations. Am Stat Assoc J 53:457-81.

Ketter TA, Post RM, Theodore WH (1999) Positive and negative psychiatric effects of antiepileptic drugs in patients with seizure disorders. Neurology 53:Suppl 2 S53-S67.

Knoester PD, Keyser A, Renier WO, Egberts ACG, Hekster YA, Deckers CLP (2005). Effectiveness of lamotrigine in clinical practice: results of a retrospective population-based study. Epilepsy Res 65:93100

Lhatoo SD, Wong IC, Polizzi G, Sander JW (2000) Long-term retention rates of lamotrigine, gabapentin, and topiramate in chronic epilepsy. Epilepsia 41:1592-6.

Luciano AL and Shorvon SD (2007) Results of treatment changes in patients with apparently drugresistant chronic epilepsy. Ann Neurol 62;375-381.

Mohanraj R, Brodie MJ (2003) Measuring the efficacy of antiepileptic drugs. Seizure 12:413-43.

Otoul C, Arrigo C, Van Rijckevorsel K, French JA (2005) Meta-analysis and indirect comparisons of levetiracetam with other second-generation antiepileptic drugs in partial epilepsy. Clin Neuropharmacol 28:72-8.

Privitera M (2006) Large clinical trials in epilepsy: funding by the NIH versus pharmaceutical industry. Epilepsy Res 68:52-6. 
Roberts GMP, Majoie HJM, Leenen LAM, Bootsma HP, Kessels AGH, Aldenkamp AP et al. (2005) Ketter's hypothesis of the mood effects of antiepileptic drugs coupled to the mechanism of action of topiramate and levetiracetam. Epilepsy \& Behavior 6:366-372.

Sander JW (2004). The use of anti-epileptic drugs - principles and practice. Epilepsia 45(Suppl. 6):2834.

Simister RJ, Sander JW, Koepp MJ. Long-term retention rates of new antiepileptic drugs in adults with chronic epilepsy and learning disability. Epilepsy Behav 10:336-9.

Smith D, Baker G, Davies G, Dewey M, Chadwick DW (1993) Outcomes of add-on treatment with lamotrigine in partial epilepsy. Epilepsia 34:312-22.

Tatum WO, French JA, Faught E et al. (2001). Postmarketing experience with topiramate and cognition. Epilepsia 42:1134-40.

Weintraub D, Buchsbaum R, Resor Jr SR, Hirsch LJ (2007) Psychiatric and behavioural side effects of the newer antiepileptic drugs in adults with epilepsy. Epilepsy Behav 10:105-10.

White JR, Walczak TS, Leppik IE et al (2003) Discontinuation of levetiracetam because of behavioral side effects: a case-control study. Neurology 61:1218-21. 



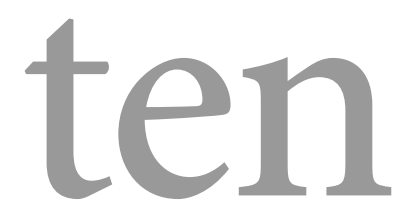

General discussion Summary

Samenvatting 



\section{General discussion}

The majority of patients with epilepsy are treated with anti-epileptic drugs (AEDs). This treatment is not without complications. Approximately $20-30 \%$ of the patients do not become seizure free on AEDs. In addition many patients suffer from side effects. Therefore it is important to find new anti-epileptic drugs and to investigate them thoroughly (Perucca et al., 2007).

New AEDs are typically tested in patients with partial epilepsies. The new AED or placebo is added to the one or two existing AEDs of the patients (add-on trial).

These regulatory trials are valuable but have serious limitations caused by the atypical patient population in the trial, the short duration of the trial and the differences between a drug trial and clinical practice. Therefore, results from regulatory trials cannot be easily translated into guidelines for clinical practice (Sander, 2005). Safety data are scarce and the comparative efficacies of the new drugs are not known.

To overcome these problems the concept of the randomized "use" trial was introduced. In this design patients are randomized to one of the new drugs, titrated to their own ideal dose, and followed over a long period of time. These trials are expensive and it is difficult to find a sponsor because the outcome is not predictable.

We decided to evaluate the long-term outcomes of three new anti-epileptic drugs: lamotrigine (LTG), levetiracetam (LEV) and topiramate (TPM) in patients of a specialized epilepsy centre. Evaluation started immediately after their introduction in clinical practice. Instead of randomization a naturalistic observational approach was chosen. After introduction, the first 300-400 patients who were prescribed the new AED for the first time, were entered in the study. Retention rate was the primary outcome measure.

Retention rate is considered to be a composite measure of drug efficacy and drug safety and expresses the willingness of patients to continue drug treatment. We compared retention rates of three new anti-epileptic drugs in the same patient 
population and conclude that it is a useful parameter which can be easily measured and reflects the use of a drug in clinical practice. Two and three year retention rates was used in all studies (Bootsma et al., 2004; 2007; 2008).

The effects of these AEDs were studied in the patient group of a tertiary referral centre and thus consisted of patients with severe refractory epilepsies. In this patient group the efficacy of the new anti-epileptic drugs appeared to be limited. Moreover retention rate appeared to be largely determined by its side effect profile. Therefore it is very important to consider the side effect profile of a new anti-epileptic drug when it is added to the medication of a patient with refractory epilepsy. For evaluation purposes it is not enough to know the retention rate. It is necessary to know the details such as the reason why a patient stops taking the drug. Is it for a lack of efficacy, or because he is experiencing side effects or both? Which side effects cause the discontinuation of the drug? Also vice versa: which side-effects or which balance between efficacy and side-effects are accepted by the patients and result in continuing drug treatment.

WHICH ANTI-EPILEPTIC DRUG IS BEST FOR AN INDIVIDUAL

PAT I E N T ?

If only retention rate is considered, lamotrigine is clearly the most favourable drug. Kaplan-Meier survival analysis, revealed significant differences between the drugs. Estimated retention rates of $75.2 \%$ (LTG), 65.6\% (LEV), and 51.7\% (TPM) were found at one year. Retention at two years had decreased to $69.2 \%$ for LTG, $45.8 \%$ for LEV, and 38.3\% for TPM. A two-year retention rate of almost $70 \%$ as obtained for lamotrigine is higher than found in most other studies for other anti-epileptic drugs (Lhatoo et al., 2000)

Surprisingly, higher seizure freedom rates were not associated with higher retention rates. The 6-months seizure freedom rates for LTG were lower than for TPM or LEV. Long-term retention for TPM was dramatically lower than for LTG, whereas seizure freedom rates were higher. This suggests that the impact of the achievement of seizure remission on long-term retention is considered less important.

On the other hand adverse events had a higher impact on drug discontinuation. As a marker of tolerability we used the percentage of patients withdrawing drug treatment because of adverse events. Adverse events played a role in drug discontinuation in $35.9 \%$ patients on TPM and in $15.5 \%$ patients on LTG and in $22.5 \%$ patients on LEV.

AED-specific adverse events that often led to discontinuation of drug treatment were mental slowing and dysphasia in patients on TPM, mood disorders in patients on LEV, and rash in patients on LTG 
We can therefore conclude that lamotrigine is a well tolerated drug. Approximately $30 \%$ of the patients on lamotrigine continued the drug while it had no effect on their seizure frequency. This was related to the occurrence of so called positive side effects. Patients on lamotrigine often report to feel more active, more clear and are more responsive.

Lamotrigine thus seems to be a good choice for patients when an activating effect on cognition or mood is desirable and/or when side effects must be avoided. The efficacy is limited, compared to topiramate and levetiracetam.

Topiramate shows the lowest retention rate. After two years $38 \%$ of the the patients still used this drug. Tolerability is a real issue for this AED : 10\% of the patients discontinued topiramate because of side effects while the drug had a clear favourable effect on seizure frequency. 3\% of the patients stopped topiramate because of side effects while being seizure free. Side effects are mentioned as a serious problem in $36 \%$ of the patients on topiramate. On the other hand our study shows excellent efficacy data for this drug. The most commonly reported side-effects are cognitive side-effects that are also responsible for the majority of the drug discontinuations.

Levetiracetam has an intermediate position in this spectrum. It combines good efficacy and limited side effects and we conclude it is a very useful anti-epileptic drug. However levetiracetam does not cause the activating effects that are reported for lamotrigine and, due to the large impact of such factors on retention, the resulting retention rate is lower than for lamotrigine.

OTHER FINDINGS ON LAMOTRIGINE, LEVETIRACETAM AND T OP I R A M A T E

\section{Side effects}

The side-effect profiles of the 3 drugs are different. For topiramate neurocognitive side effects are the most frequent reason for drug discontinuation. A novel observation in our study is that slower titration, a low starting dose and a lower maintenance dose cannot prevent these side effects. For levetiracetam mood disorders are the most important side effects leading to discontinuation of the drug. For lamotrigine dizziness was the most reported side effect in the patients who discontinued treatment. 


\section{Titration schedules}

Our study showed that in clinical practice fixed strategies for dose titration are seldom used. In contrast, an individualized approach is chosen in response to patient complaints and seizure frequency.

For topiramate the measured mean titration dose ( $25 \mathrm{mg} /$ week) follows the current recommendations. This slow dose escalation however did not prevent the occurrence of cognitive side effects and did not improve retention rate.

The ease in titrating levetiracetam is well illustrated by the finding that the most cautious titration schedule was not applied in a single patient. Often faster titration schedules were used than recommended by the manufacturer.

For lamotrigine titration is dependent on comedication. It is generally known that slow titration helps to prevent dermatologic side effects.

\section{Prescribed daily dose}

In clinical practice the prescribed daily dose of topiramate was $200-300 \mathrm{mg}$. This was much lower than the $600 \mathrm{mg} /$ day which was recommended in the product information at that time but is in line with current medical practice.

Levetiracetam dosing in clinical practice was in accordance with the recommendations of the manufacturer. Very soon after the start of levetiracetam a dose between 2000 and $2500 \mathrm{mg} /$ day was reached.

For lamotrigine many patients got higher dosages than recommended by the manufacturer.

\section{Drug-interactions}

The more frequent use of lamotrigine and vigabatrin as comedication in the patients who stopped taking topiramate is interesting but is difficult to interpret in an observational study. The pharmacokinetic interactions between lamotrigine and valproate and between lamotrigine and enzyme-inducing anti-epileptic drugs could be affirmed.

Retention rates could have been influenced by the order in which LTG, LEV, and TPM were introduced in clinical practice. Lamotrigine was introduced first in clinical practice (in 1996), topiramate was introduced in 1999 and levetiracetam in 2001. Patients on drugs that were introduced first could have been withdrawn from 
treatment because of the availability of a new AED. On the other hand, drugs that were introduced later could have been tested in a more refractory population as only those who did not respond to earlier new drugs were available. We have not seen such effects as the population in which the three drugs were tested were similar but we can not exclude such effects completely.

\section{WHAT LESSONS CAN BE LEARNED}

Drug trials do not provide sufficient information to use newer AEDs in clinical practice. Especially the long-term effects of a drug are unknown at the time of introduction in clinical practice. This implies that issues such as: is it possible that tolerance develops (thus: is the efficacy of the drug limited in time), which side-effects occur over time (and which side-effects disappear), what is the correct dose at long-term, what interactions occur with concomitant treatments.

It is important that such information is available shortly after introduction of a new AED in clinical practice. We had to analyse the data that provide such information using different sources in a very time-consuming effort.

It will be extremely helpful if we are able to measure retention times for a new drug automatically in medical information systems or electronic patient files. Such recordings provide for a database that can generate the necessary data within one or two years after introducing the drug. To avoid bias, data collection should involve every patient starting the new drug (Faught et al., 2008).

Our studies provide the necessary data that should be recorded and therefore should be included in the electronic systems:

- Retention time in months

- Seizure recordings (especially periods seizure free). Preferably some form of patient calendar should be available to have continuous data (in the absence of such a system, the neurologist will often record non-continuous data such as 'doing better' or 'does not improve').

- Spontaneously reported side effects

- Observed side-effects

- Reason for withdrawal: Lack of efficacy/ Side effects/ Both

- Which side-effect is the reason for stopping?

- Other medications and total drug load.

Most electronic patient files will already generate such data, allowing linkage with multicentre databases for evaluation. 
The set up of this thesis and the studies carried out, started with the idea of a hospital pharmacist. It was thought that the patient records available in the medical and pharmaceutical databases in our epilepsy center might help us to make rational choices between new anti-epileptic drugs. The idea was adopted by our drug research group and due to the co-operation of this multidisciplinary team the project could be performed. This is an example of a contribution of a hospital pharmacist to the care for patients suffering from refractory epilepsy: contributing in a systematic process of collecting data that can improve clinical practice.

There are other options for a hospital pharmacist to contribute in this patient group.

Hospital pharmacists aim at best clinical use of medication for the patients. We once admitted a patient who used an oral suspension of oxcarbazepine. This product could not be resuspended properly which led to underdosing of the drug. The patients epilepsy deteriorated and it took 3 months to get it under control again. Changing to the "syringe method" solved this problem. Hospital pharmacists can help by changing the formulation of a drug product e.g. midazolam intranasal spray as an alternative for rectal diazepam (Knoester et al., 2002) or they can give advice on generic substitution in the treatment of epilepsy (Berg et al., 2008).

Patients with refractory epilepsy often use enzyme-inducing anti-epileptic drugs. In fact $75 \%$ of our inpatients use an enzyme-inducing AED. These drugs alter the pharmacokinetics of numerous other drugs. The hospital pharmacist can make a special formulary for this patient population (Bootsma, 2002). The formulary can also take into account that some drugs are contra-indicated in epilepsy.

Hospital pharmacists are trained to provide a therapeutic drug monitoring (TDM) service. In this way we can help neurologists to find the best AED dose for a patient. There is still a need for studies assessing the impact of TDM on the outcome of treatment of epilepsy (Patsalos et al., 2008).

A new field to explore is pharmacogenetics. Pharmacists can measure (multidrug resistance) genes and try to correlate gene profiles to AED drug response.

Hospital pharmacists are in an ideal position to acquire knowledge on side effects of drugs and to distribute this knowledge. And there is the problem of non-adherence to anti-epileptic drugs. In the RANSOM study pharmacy claims were used to measure adherence to prescriptions. Non-adherence led to the increased mortality and morbidity of epilepsy (Faught et al., 2008). Pharmacists must develop strategies to improve adherence to anti-epileptic drug regimens and they are in the position to do so.

Our study shows the possibilities of joint efforts in a multidisciplinary team to the benefit of patient treatment. 


\section{R E F E R E N C E S}

Berg MJ, Gross RA, Tomaszewski KJ et al. Generic substitution in the treatment of epilepsy. Case evidence in breaktrough seizures. Neurology 2008;71:525-530.

Bootsma HPR Anti-epileptic drugs and co-medication: suggestions for clinical practice. Acta Neurol. Belg. 2002(3):144 Abstract

Bootsma HP, Coolen F, Aldenkamp AP, Arends J, Diepman L, Hulsman J, Lambrechts D, Leenen L, Majoie M, Schellekens A, de Krom M. Topiramate in clinical practice: long-term experience in patients with refractory epilepsy referred to a tertiary epilepsy center. Epilepsy Behav. 2004 Jun;5(3):380-7.

Bootsma HP, Ricker L, Diepman L, Gehring J, Hulsman J, Lambrechts D, Leenen L, Majoie M, Schellekens A, de Krom M, Aldenkamp AP. Levetiracetam in clinical practice: long-term experience in patients with refractory epilepsy referred to a tertiary epilepsy center. Epilepsy Behav. 2007 Mar;10(2):296303.

Bootsma HP, Vos AM, Hulsman J, Lambrechts D, Leenen L, Majoie M, Savelkoul M, Schellekens A, Aldenkamp AP. Lamotrigine in clinical practice: long-term experience in patients with refractory epilepsy referred to a tertiary epilepsy center. Epilepsy Behav. 2008 Feb;12(2):262-8.

Faught E, Duh MS, Weiner JR, Guérin A, Cunnington MC. Nonadherence to antiepileptic drugs and increased mortality: findings from the RANSOM Study. Neurology. 2008 Nov 11;71(20):1572-8.

Knoester PD, Jonker DM, Van der Hoeven RTM, et al. Pharmacokinetics and pharmacodynamics of midazolam administered as a concentrates intranasal spray. A study in healthy volunteers. Br J Clin Pharmacol 2002;53:501-7

Lhatoo SD, Wong IC, Polizzi G, Sander JW. Long-term retention rates of lamotrigine, gabapentin, and topiramate in chronic epilepsy. Epilepsia 2000;41:1592-6.

Patsalos PN, Berry DJ, Bourgeois BFD et al. Antiepileptic drugs - best practice guidelines for therapeutic drug monitoring: A position paper by the subcommission on therapeutic drug monitoring, ILAE Commission on Therapeutic Strategies. Epilepsia 2008;49(7):1239-1276.

Perucca E, French J, Bialer M. Development of new antiepileptic drugs: challenges, incentives, and recent advances. Lancet Neurol. 2007 Sep;6(9):793-804.

Sander JW New antiepileptic drugs in practice. How do they perform in the real world? Acta Neurol Scand Suppl. 2005;181:26-9 



\section{Summary}

There are about 100,000 patients with epilepsy in the Netherlands. For almost all these patients drug therapy is the mainstay of their treatment. The aim of drug therapy is to prevent the occurrence of seizures completely. However, approximately 20-30\% of the patients do not become seizure-free on anti-epileptic drugs (AEDs). In addition many patients suffer from side effects. Therefore it is important to find new drugs.

Patients who do not become seizure free on two, or more than two AEDs, are called refractory patients or pharmacoresistant patients. This patient category was involved in our long-term studies on three new anti-epileptic drugs: topiramate (TPM), levetiracetam (LEV) and lamotrigine (LTG). For a good interpretation of the results presented in this thesis it is important to realise that the likelihood to respond to drug therapy is a function of the past treatment history. For every two anti-epileptic drugs that proved ineffective in the past, the percentage of patients who respond with a greater than $50 \%$ reduction in seizure frequency decreases by twofold.

In chapter 2 the pros and cons of regulatory clinical trials are described. We performed a systematic search of the literature on clinical trial designs and outcome measures for the evaluation of AEDs. We found that the anti-epileptic drug approval process is based primarily on short-term, randomized, placebo-controlled, parallel-group, add-on studies. These studies find anticonvulsive drugs which are more effective than placebo but cannot tell us which new anti-epileptic drug will be best in clinical practice. Regulatory trials are necessary to prove the efficacy and the safety of new AEDs but long-term studies may add valuable information as is pointed out in this chapter. Important efficacy parameters for long-term studies are seizure freedom and retention rate. Also much more attention should be given to the monitoring of side effects because tolerability problems often lead to discontinuation of AED therapy. 
In chapter 3 we describe our experience with the first 470 patients who were treated with topiramate in the Epilepsy Centre Kempenhaeghe. We followed these patients for 4 years and used the retention rate as primary outcome parameter. In clinical practice the prescribed daily dose was $200-300 \mathrm{mg}$. This was much lower than the $600 \mathrm{mg} /$ day which was recommended in the product information at that time but is in line with current medical practice.

The effects of TPM on seizure frequency were rather impressive in our refractory patients. In 6-months evaluations about $10-15 \%$ of the patients became seizure-free. In approximately $30 \%$ of the patients the drug had a favourable effect on seizure frequency.

For topiramate the neurocognitive side effects were the most frequent reason for drug discontinuation. We found that a low starting dose, a slower titration and a lower maintenance dose cannot prevent these side effects. In about $10 \%$ of the patients side effects led to discontinuation despite the obvious favourable effects on seizure frequency. Retention rate was 53\% after 1 year, $45 \%$ after 2 years, 38\% after 3 years and $30 \%$ after 4 years.

The main reasons for drug discontinuation were side effects. In $23 \%$ of the patients side effects were the only reason for discontinuation. $14 \%$ of the patients experienced side effects and a lack of efficacy of TPM and therefore decided to discontinue the drug. Therefore, side effects played a role in discontinuation of TPM in almost $40 \%$ of the total study group. We did not find meaningful prognostic factors that may help us in clinical decision making, i.e. help us to prevent those side effects or help us to identify those at risk.

Our conclusion is that topiramate is associated with a high incidence of side effects in clinical practice which affects long-term retention.

In chapter 4 we report on our levetiracetam (LEV) study. In total 301 patients were included and followed for 2 years. The clinical dose achieved in our population was 2000-2500 mg/day (adults only) and this was reached within a few weeks. The mean titration dose was $454 \mathrm{mg} /$ week. These findings are in accordance with the recommendations of the manufacturer of LEV.

Compared with other new AEDs, the effects of LEV on seizure frequency are promising. About $15 \%$ of patients in this refractory group have a 3 -month remission, whereas $10 \%$ of patients become seizure-free for longer periods.

The percentages of patients with a mental handicap achieving seizure remission is low.

The most frequently reported side effects at the time of discontinuation were mood disorders - both activating (13.8\%) and deactivating (13.1\%) - tiredness (13.8\%) and sleepiness (8.5\%). 
The retention rate was $66 \%$ after 1 year and $46 \%$ after 2 years. Retention rates for patients with and without a mental handicap were the same.

No variables predicting (dis)continuation can be identified. Only major differences were seen in the outcome variables seizure reduction and side effects.

Our conclusion is that levetiracetam appears to be a useful AED. Its side effect profile is mild, with mood disorders being the most important side effects.

In chapter 5 the results of the lamotrigine study are presented. We included 314 patients in this study and followed them for 4 years. Many dosages given in this study were higher than recommended by the manufacturer of LTG. Higher-than-recommended (100-500 mg) dosages were prescribed for $42 \%$ of the patients on monotherapy. For patients taking LTG with an inducer $62 \%$ got a higher dose than recommended (200$400 \mathrm{mg}$ ). For patients taking LTG with valproate $63 \%$ was prescribed a higher dose than recommended (100-200 $\mathrm{mg}$ ).

Plasma levels were measured and $2.5 \%$ were above the recommended upper limit of $14 \mathrm{mg} / \mathrm{L} .26 \%$ of the plasma-levels were lower than the recommended lower limit of $3 \mathrm{mg} / \mathrm{L}$. In $85 \%$ of the plasma-levels $<3 \mathrm{mg} / \mathrm{L}$ patients were taking LTG with an enzyme-inducing AED.

In 6-months evaluations about $10 \%$ of the patients became seizure-free. In approximately $20 \%$ of the patients LTG had a favourable effect on seizure frequency.

The most frequently reported side effect at discontinuation was dizziness (15.7\%). Headache was the second most frequently reported side effect (8.4\%). However the mean reason for discontinuation of LTG was a lack of efficacy (19.1\%). During the whole study $12-15 \%$ of the patients felt more active. This can be considered as a "positive" side effect of the drug.

The retention rate was $74 \%$ after 1 year, $69 \%$ after 2 years, $63 \%$ after 3 years and $56 \%$ after 4 years.

Our conclusion is that lamotrigine is a drug that is tolerated well by our patients.

In chapter 6 we compared levetiracetam and topiramate. The retention rate for LEV was significantly higher than for TPM. LEV had a more favourable side effect profile than TPM with comparable efficacy. Patients on TPM discontinued treatment mainly because of neurocognitive side effects. In the treatment with LEV, the effects on mood must not be underestimated.

In chapter 7 we focused on the effects of levetiracetam and topiramate on cognition. Cognitive complaints are common in TPM treatment and frequently lead to drug withdrawal. The impact of LEV on cognitive function is only mild. This leads to a $15 \%$ higher drug discontinuation rate for TPM compared to LEV. 
In chapter 8 we studied the effects of levetiracetam and topiramate on mood. We tested Ketter's hypothesis that mood-modulating profiles of AEDs can be divided into 2 classes: the first is assumed to have deactivating effects related to GABA potentiation, and the second class is assumed to have activating effects that are associated with glutamate attenuation. We found LEV to manifest activating and deactivating side effects equally and TPM to act as a deactivating AED with tiredness/sleepiness side effects being predominant.

In chapter 9 we compare the retention rates of lamotrigine, levetiracetam and topiramate. Two-year retention rates were 69\% (LTG), 46\% (LEV) and 38\% (TPM). Seizure freedom rates were lowest for lamotrigine and highest for levetiracetam. Adverse events played a role in drug discontinuation in $16 \%$ of the patients on LTG, in $23 \%$ of the patients on LEV and in 40\% of the patients on TPM. Mood disorders and general CNS-effects are common in patients on LTG and LEV, and neurocognitive side effects are most prevalent on topiramate. A positive effect on cognition is frequently noted in patients on lamotrigine.

We conclude that a drug that is only modestly efficacious but has a favourable safety profile (e.g. LTG) may look better in retention time analysis than a drug that is more efficacious but produces clinically meaningful adverse events (e.g. TPM). Therefore, in this refractory patient group, a drug's retention rate is mainly determined by its side effect profile.

In the general discussion we try to translate our findings into recommendations for clinical practice. We discuss which anti-epileptic drug is best for an individual patient.

Also we propose "a minimal data set" which can become a part of electronic patient files for epilepsy patients in order to monitor our experience with new AEDs "on line". I hope we can make this work in the near future.

Hospital pharmacists aim at the best clinical use of medication for their patients. In an epilepsy centre there is a lot to do for a hospital pharmacist of which examples are given. 


\section{Samenvatting}

Er zijn ongeveer 100.000 patiënten met epilepsie in Nederland. Voor bijna al deze patiënten vormt de behandeling met geneesmiddelen het belangrijkste element van hun anti-epileptische behandeling. Het doel van de behandeling met geneesmiddelen is volledige remissie van epileptische aanvallen. Echter, ongeveer $20-30 \%$ van de patiënten worden niet aanvalsvrij door gebruik van anti-epileptica (AE). Bovendien hebben veel patiënten last van bijwerkingen. Daarom is het belangrijk om nieuwe geneesmiddelen te blijven ontwikkelen.

Patiënten die niet aanvalsvrij worden op twee of meer anti-epileptica worden refractaire of farmacoresistente patiënten genoemd. Deze categorie patiënten was betrokken bij onze lange-termijn onderzoeken met drie nieuwe anti-epileptica: topiramaat (TPM), levetiracetam (LEV) en lamotrigine (LTG). Voor een goede interpretatie van de resultaten, die in dit proefschrift worden gegeven, is het belangrijk dat men zich realiseert dat de kans om goed te reageren op anti-epileptica afhankelijk is van de behandelhistorie. Voor elke twee anti-epileptica die in het verleden ineffectief zijn gebleken halveert het percentage patiënten dat reageert met een aanvalsreductie van meer dan $50 \%$.

In hoofdstuk 2 worden de voor- en nadelen van registratie-onderzoeken beschreven. Wij voerden een systematisch literatuuronderzoek uit naar onderzoeksmethoden en uitkomstvariabelen voor klinisch onderzoek met anti-epileptica. Wij stelden vast dat het oordeel van de registratie-autoriteiten voornamelijk gebaseerd is op kortetermijn, gerandomiseerde, placebo-gecontroleerde, parallelgroep, add-on studies. Dit type onderzoek vindt anticonvulsieve geneesmiddelen die effectiever zijn dan een placebo maar zij kunnen ons niet vertellen welk nieuw anti-epilepticum het beste zal zijn in de klinische praktijk. Registratie-onderzoeken zijn nodig om de effectiviteit en de veiligheid van nieuwe anti-epileptica vast te stellen maar lange-termijn studies kunnen belangrijke informatie toevoegen zoals wordt aangegeven in dit hoofdstuk. 
Belangrijke maten voor de effectiviteit in lange-termijn studies zijn aanvalsvrijheid en retentie. Verder moet er veel meer aandacht worden gegeven aan het bespreken en registreren van bijwerkingen omdat deze vaak leiden tot het stoppen van de behandeling met een anti-epilepticum.

In hoofdstuk 3 beschrijven wij onze ervaringen met de eerste 470 patiënten die werden behandeld met topiramaat in het Epilepsiecentrum Kempenhaeghe. Wij volgden deze patiënten gedurende 4 jaar en gebruikten de langere-termijn retentie als de primaire uitkomstvariabele. In de klinische praktijk was de voorgeschreven dagelijkse dosering 200-300 mg. Dit was veel lager dan de $600 \mathrm{mg} / \mathrm{dag}$ die werd aanbevolen in de bijsluiter op dat moment maar was in overeenstemming met de doseringen die elders werden gebruikt.

De effecten van topiramaat op de aanvalsfrequentie waren nogal indrukwekkend in onze refractaire patiëntengroep. $10-15 \%$ van de patiënten werden aanvalsvrij (waarbij een termijn van 6 maanden werd gehanteerd). In ongeveer $30 \%$ van de patiënten had het geneesmiddel een gunstig effect op de aanvalsfrequentie.

De cognitieve bijwerkingen van topiramaat werden het meest genoemd als reden om weer met het geneesmiddel te stoppen. Wij stelden vast dat een lage startdosering, een langzamer titratieschema en een lagere onderhoudsdosering het optreden van deze bijwerkingen niet kan voorkomen. Bij ongeveer $10 \%$ van de patiënten leidden bijwerkingen tot het stoppen van topiramaat ondanks het gunstige effect dat het geneesmiddel had op de aanvalsfrequentie. De retentie was $53 \%$ na 1 jaar, $45 \%$ na 2 jaar, $38 \%$ na 3 jaar en $30 \%$ na 4 jaar.

De belangrijkste redenen voor het staken van de behandeling waren bijwerkingen. Voor 23\% van de patiënten waren bijwerkingen de enige reden voor het stoppen van topiramaat. $14 \%$ van de patiënten gaf aan bijwerkingen te hebben en geen gunstig effect te ondervinden en stopte daarom met de behandeling. Dus bijwerkingen speelden een rol als stopreden bij bijna $40 \%$ van de gehele patiëntengroep. Wij vonden geen betekenisvolle prognostische indicatoren die ons zouden kunnen helpen bij klinische besluitvorming, bijvoorbeeld factoren die ons kunnen helpen bij het voorkomen van bijwerkingen of factoren die patiënten kunnen identificeren die een hoger risico hebben op bijwerkingen.

Wij concluderen dat topiramaat veel bijwerkingen geeft in de klinische praktijk en dat daardoor de langere-termijn retentie negatief wordt beïnvloed.

In hoofdstuk 4 rapporteren wij over onze levetiracetam studie. In het totaal werden 301 patiënten geincludeerd en gedurende 2 jaar gevolgd. De onderhoudsdosering die werd bereikt in onze populatie bedroeg 2000-2500 mg/dag (voor volwassenen) en deze dosering werd binnen enkele weken bereikt. Bij het insluipen was de gemiddelde 
dosisverhoging $454 \mathrm{mg} /$ week. Deze bevindingen waren in overeenstemming met de aanbevelingen van de fabrikant van levetiracetam.

Vergeleken met de andere nieuwe anti-epileptica waren de effecten van levetiracetam op de aanvalsfrequentie veelbelovend. Ongeveer $15 \%$ van de patiënten in deze refractaire groep werd aanvalsvrij (hier werd een periode van 3 maanden gehanteerd), terwijl 10\% van de patiënten gedurende een langere periode aanvalsvrij bleef.

Van de patiënten met een verstandelijke handicap werd een veel kleiner percentage aanvalsvrij.

De meest voorkomende bijwerkingen op het moment van stoppen waren stemmingsstoornissen - zowel activerende $(13,8 \%)$ als deactiverende $(13,1 \%)$ - vermoeidheid $(13,8 \%)$ en slaperigheid $(8,5 \%)$.

De retentie was $66 \%$ na 1 jaar en $46 \%$ na 2 jaar. De retentie voor patiënten met en zonder verstandelijke handicap waren gelijk.

Wij konden geen factoren vinden die een verband hadden met het stoppen van levetiracetam. Er werden alleen grote verschillen gezien in de uitkomstvariabelen aanvalsreductie en bijwerkingen.

Wij concluderen dat levetiracetam een bruikbaar anti-epilepticum is. Het bijwerkingenprofiel is mild waarbij stemmingsstoornissen de belangrijkste bijwerkingen zijn.

In hoofdstuk 5 worden de resultaten van de lamotrigine studie gepresenteerd. Wij includeerden 314 patienten in deze studie en volgden ze gedurende 4 jaar. In deze studie werd vaak hoger gedoseerd dan de aanbevolen dosering van de fabrikant. Voor monotherapie werd een dosering tussen de 100-500 mg aanbevolen maar 42\% van de patiënten kreeg een hogere dosering. Van de patiënten die naast lamotrigine ook een enzym-inducerend anti-epilepticum slikten kreeg $62 \%$ een hogere dosering dan in de bijsluiter werd aanbevolen (200-400 mg). Ook voor 63\% van de patiënten die zowel lamotrigine als valproaat kregen was de dosering hoger dan aanbevolen (100-200 $\mathrm{mg})$.

Wij hebben plasmaspiegels van lamotrigine gemeten en $2,5 \%$ van de metingen bleek hoger te zijn dan de aanbevolen bovengrens van $14 \mathrm{mg} / \mathrm{L} .26 \%$ van de gemeten spiegels waren lager dan de aanbevolen ondergrens van $3 \mathrm{mg} / \mathrm{L}$. In $85 \%$ van deze lage meetwaarden ging het om patiënten die naast lamotrigine ook een enzym-inducerend anti-epilepticum gebruikten.

Ongeveer 10\% van de patiënten werd aanvalsvrij gedurende een periode van 6 maanden. In ongeveer $20 \%$ van de patiënten gaf lamotrigine een gunstig effect op de aanvalsfrequentie.

De meest gerapporteerde bijwerking op het moment van stoppen met lamotrigine was duizeligheid (15,7\%). Op de tweede plaats kwam hoofdpijn $(8,4 \%)$. Echter de belang- 
rijkste reden voor het staken van lamotrigine was het gebrek aan effectiviteit (19,1\%). Gedurende de gehele studie voelden $12-15 \%$ van de patiënten zich meer actief. Dit zou beschouwd kunnen worden als een positieve bijwerking van dit geneesmiddel.

De retentie was $74 \%$ na 1 jaar, $69 \%$ na 2 jaar, $63 \%$ na 3 jaar en $56 \%$ na 4 jaar. Wij concluderen dat lamotrigine een geneesmiddel is dat in het algemeen goed wordt verdragen door onze patiënten.

In hoofdstuk 6 hebben wij levetiracetam en topiramaat met elkaar vergeleken. De retentie voor levetiracetam was significant hoger dan die voor topiramaat. Levetiracetam had een gunstiger bijwerkingenprofiel dan topiramaat terwijl de middelen ongeveer even effectief bleken te zijn. Patienten die topiramaat gebruikten stopten vooral vanwege het optreden van cognitieve bijwerkingen. Bij de behandeling met levetiracetam moeten de effecten op de stemming niet onderschat worden.

In hoofdstuk 7 richtten wij ons op de effecten van levetiracetam en topiramaat op cognitie (kenvermogen). Cognitieve bijwerkingen komen veel voor bij de behandeling met topiramaat en leiden vaak tot staken van het geneesmiddel. Het effect van levetiracetam op de cognitieve functie is daarentegen gering. In onze studie stopten $54 \%$ van de patiënten die topiramaat gebruikten tegenover $39 \%$ van de patiënten die levetiracetam gebruikten in een behandelperiode van 18 maanden (dus 15\% meer stoppers door topiramaat).

In hoofdstuk 8 bestudeerden wij de invloed van levetiracetam en topiramaat op stemming. Wij testen Ketter's hypothese dat anti-epileptica, voor wat hun invloed op de stemming betreft, in 2 klassen ingedeeld kunnen worden: de eerste klasse wordt verondersteld deactiverende effecten te hebben door potentiering van GABA, en de tweede klasse wordt verondersteld activerende effecten te hebben door verzwakking van het effect van glutamaat. Wij vonden dat levetiracetam in gelijke mate zowel activerende als deactiverende eigenschappen had. Topiramaat daarentegen had deactiverende eigenschappen waarbij vooral vermoeidheid en slaperigheid als bijwerkingen optraden.

In hoofdstuk 9 vergelijken wij de retentiewaarden van lamotrigine, levetiracetam en topiramaat. De retentiewaarden waren na 2 jaar 69\% (LTG), 46\% (LEV) and 38\% (TPM). Het percentage patiënten dat aanvalsvrij werd was het laagste voor lamotrigine en het hoogste voor levetiracetam. Bijwerkingen speelden een rol bij het staken van de behandeling bij $16 \%$ van de patiënten op lamotrigine, bij $23 \%$ van de patiënten op levetiracetam en bij $40 \%$ van de patiënten op topiramaat. Stemmingsstoornissen en algemene bijwerkingen op het zenuwstelsel kwamen veel voor bij patiënten op lamotrigine en levetiracetam en cognitieve bijwerkingen kwamen het meest voor 
bij het gebruik van topiramaat. Een positief effect op cognitieve functies werd vaak gezien bij patiënten die lamotrigine gebruikten.

Wij concludeerden dat een geneesmiddel dat slechts matig effectief is maar een gunstig veiligheidsprofiel heeft (bv LTG) leidt tot een hogere retentiewaarde dan een geneesmiddel dat een beter effect heeft op de epilepsie maar daarnaast hinderlijke bijwerkingen geeft (bv TPM). Daarom wordt, in deze refractaire patiëntengroep, de retentiewaarde van een anti-epilepticum vooral bepaald door het bijwerkingenprofiel.

In de algemene discussie proberen wij onze bevindingen te vertalen in aanbevelingen voor de klinische praktijk. Wij gaan in op de vraag welk van deze nieuwe anti-epileptica het beste voorgeschreven kan worden aan een bepaalde patiënt. Verder stellen wij een "minimale gegevensverzameling" voor die deel zou kunnen worden van een elektronisch patiëntendossier voor epilepsiepatienten met het doel onze ervaringen met nieuwe anti-epileptica "on line" te kunnen volgen.

Ik hoop dat wij dit spoedig kunnen gaan implementeren.

Ziekenhuisapothekers stellen zich tot doel dat hun patiënten een optimaal resultaat krijgen met hun geneesmiddelen. In een epilepsiecentrum is er van alles te doen voor een ziekenhuisapotheker. Aan het einde van de algemene discussie worden daar nog wat voorbeelden van gegeven. 



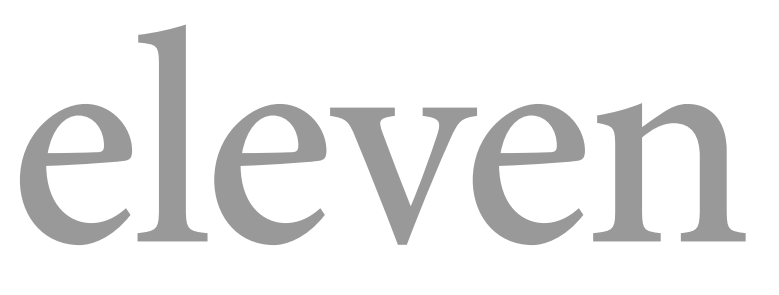

Dankwoord

Curriculum vitae

List of publications 



\section{Dankwoord}

Allereerst gaat mijn dank uit naar mijn promotoren en mijn copromotor. Beste Bert (Aldenkamp), jouw enorme ervaring met epilepsie-onderzoek en het begeleiden van promoties hebben dit onderzoek in een stroomversnelling gebracht. Het idee waar $\mathrm{ik}$ al langer mee rondliep kreeg door jouw bemoeienis echt vorm. Altijd weer wist jij bij onze werkbesprekingen snel door te dringen tot de kern. Daardoor was er ook altijd tijd voor koffie en een gesprek over onze kinderen. Door jouw "doen we even" mentaliteit wist jij de grizzlyberen op mijn pad weer om te toveren tot teddyberen. Ook bewonder ik het geduld dat je met deze parttime promovendus had.

Beste Chiel (Hekster), jij was onmiddellijk enthousiast toen ik al weer bijna 10 jaar geleden in Nijmegen bij je op bezoek kwam. Met je priemende ogen hoorde je me eerst uit over mijn plannen om vervolgens een stortvloed aan ideeën met mij te delen. In die fase vond ik het nog wel heel lastig om de plannen en ideeën om te zetten in daden. Ook achteraf kan ik vaststellen dat ik lang niet al jouw goede ideeën heb kunnen uitvoeren. Maar jij bleef enthousiast en vooral in de laatste fase zeer dichtbij via ons e-mail verkeer.

Beste Jacques (Hulsman), wij kenden elkaar al langer van onze werkzaamheden in het St Anna ziekenhuis. Jouw bereidheid tot multidisciplinaire samenwerking was altijd groot. Jij deed al onderzoek in Kempenhaeghe toen er nog geen echte infrastructuur voor was en je beschikt over een echte pioniersmentaliteit. Terwijl anderen soms gaan afbouwen als het pensioen in zicht komt gooi jij er juist een schepje bovenop. Ook was jij altijd bereid "mijn" studenten te huisvesten binnen het laboratorium en ze van goede raad te voorzien. Ook jouw medewerkers Anouk Bol, Marlie van Bree, Ton Broekman, Els Floor, Meri-An Kastelijn, Vedrana Nikolic, Lilian Pompen, Adriaan Schellekens, Corine Vogels en Thea Weiss hebben mij en en "mijn" studenten altijd zeer gastvrij ontvangen en geholpen waar mogelijk.

Ike Bomer en Paul Boon die verantwoordelijk zijn voor de bedrijfsvoering en de wetenschappelijke ontwikkeling van het epilepsiecentrum Kempenhaeghe wil ik 
bedanken voor het creëren van een werkomgeving waarin professionals kunnen doen waar ze goed in zijn en een omgeving waarin professionals steeds beter kunnen worden en wel op een zodanige manier dat het direct ten goede kan komen van de dagelijkse patiëntenzorg.

Al het geneesmiddelenonderzoek in Kempenhaeghe wordt gecoördineerd door de multidisciplinair samengestelde geneesmiddelengroep. De leden van deze groep (Bert Aldenkamp, Ariadne van der Does, Jacques Hulsman, Danielle Lambrechts, Loes Leenen, Marian Majoie, Petra van Mierlo, Marion Savelkoul, Adriaan Schellekens en Pauline Verschuure) hebben mij de ruimte gegeven om dit onderzoek te doen en hebben mij geholpen met goede ideeën en het interesseren van alle neurologen voor mijn vraagstelling. Ik ben jullie allemaal veel dank verschuldigd en Danielle en Marian in het bijzonder. Ook de inbreng van onze liaison in Maastricht Marc de Krom heb ik zeer gewaardeerd.

Dit onderzoek had niet afgerond kunnen worden zonder de hulp van "mijn" studenten Frank Coolen, Lukas Ricker, Astrid Vos en Loes Jacobs. Samen hebben wij veel geleerd. Ik vind het geweldig dat jullie allemaal een prachtige carrière tegemoet gaan en dat Lukas ook zal promoveren.

I also want to thank Gloria Roberts for her help with the data collection in the topiramate project and the levetiracetam project. All the way from Ireland you studied our medical files which were written in the Dutch language! Your work resulted in a fine paper on the Ketter's hypothesis (chapter 8 of this thesis) and at the moment you are working on a thesis yourself. Marian Majoie is de motor geweest achter dit project waarvoor dank.

De hulp van Loes Leenen en Leonie Diepman bij het verwerken van onderzoeksgegevens heb ik ook erg gewaardeerd. Zij schoten te hulp als ik dat echt even nodig had. Ook zeer veel dank aan alle neurologen van Kempenhaeghe die hun patiënten-gegevens met mij wilden delen: Johan Arends, Albert Colon, Mariette Debeij-van Hall, Jan Doelman, Saskia Ebus, Gerard van Erp, Guus Janssen, Bert Kornips, Danielle Lambrechts, Richard Lazeron, Anton de Louw, Marian Majoie, dr Oei, Theo Rentmeester en Rob Starrenburg.

Een speciaal woord van dank voor Paul Griep en zijn medewerkers van de automatiseringsafdeling. Jullie waren altijd bereid mee te denken over "queries" en doordat jullie al jaren geleden begonnen zijn met een infrastructuur voor het systematisch verzamelen van informatie, beschikken wij over betrouwbare gegevens voor onderzoek.

Tenslotte wil ik dan toch stilstaan bij mijn thuisfront. Als je op verkenning gaat is het goed af en toe weer een schuilplaats op te kunnen zoeken. Eén van de schuilplaatsen werd gevormd door onze vakgroep van ziekenhuisapothekers met Christian, Emmeke en Janneke die altijd belangstelling voor het onderzoek hebben getoond en mij hebben geholpen waar dat mogelijk was. De andere schuilplaats is mijn beste schuilplaats maar die houd ik geheim. 


\section{Curriculum vitae}

Hans-peter Bootsma was born on march 14 st , 1960 in Hoogeveen, The Netherlands. He grew up in Utrecht and Bunnik and followed a Gymnasium $\beta$ education at College Blaucapel in Utrecht from 1972 till 1978. He studied pharmacy at Groningen University. He obtained his MSc degree in 1985 and his PharmD degree in 1986. Then he was appointed as an assistant-researcher in the field of pharmaceutical technology at Groningen University under the supervision of prof. dr. ir. Lerk. He started his training to become a hospital pharmacist in 1988 in the Scheper hospital in Emmen under the supervision of Martien van de Poll and Willem Boeke. At the same time he teached PharmD students at Groningen University.

In 1992 he was appointed as a hospital pharmacist in the St Anna hospital in Geldrop and joined his collegue Leo Tillmanns. In 1997 he also started working on a part-time basis in the Epilepsy Center Kempenhaeghe. In 2003 he was appointed as the Pharmacy Director in the St Anna hospital.

The studies for this thesis were carried out in the Epilepsy Center Kempenhaeghe in Heeze, the Netherlands.

Hans-peter Bootsma is married to Harmienke Kloeze. They have two daughters (Nynke and Sietske) and a son (Sjoerd). 


\section{List of publications}

Bootsma HPR, Neutel JW, van der Sluijs P, Meyer DKF, Molenaar F Lactosylated albumin: a potential carrier for drug delivery to hepatocytes. Pharm. Weekblad [Sci] $1985 ; 7: 28$

Bootsma HPR, van der Sluijs P, Molenaar F, Meyer DKF Hepatic disposition of lactosylated-albumins: potential carriers for drug targeting to the liver. J. Hepatol. 1985;1(Suppl.2):S196, Abstract

van der Sluijs P, Bootsma HPR, Postema B, Molenaar F, Meyer DKF Drugtargeting to the liver with lactosylated albumins: does the glycoprotein target the drug or is the drug targeting the glycoprotein? Hepatology 1986;6(4):723-8

Bootsma HPR, Frijlink JW, Eissens A, Proost JH, van Doorne H, Lerk CF Betacyclodextrin as an excipient in solid oral dosage forms: in vitro and in vivo evaluation of spray-dried diazepam- $\beta$-cyclodextrin products. Int. J. Pharm. 1989;51;213-23

Bootsma HPR, Kaufmann BG, Boeke AW, van de Poll MAPC De afgifte van silicaat uit infuusflessen. Ziekenhuisfarmacie 1991;7(4):113-7

de Weerdt CJ, Bootsma HPR, Hendriks H Herbal medicines in migraine prevention. Randomised double-blind placebo-controlled crossover trial of a feverfew preparation. Phytomedicine 1996;III(3):225-30

Bootsma HPR teksten voor CD-rom Medicijnen (2000) in samenwerking met Transferpunt Vaardigheidsonderwijs Maastricht Bohn Stafleu van Loghum ISBN 90313 26372 (wordt gebruikt bij opleidingen voor verpleegkunde) 
Bootsma HPR, Carbamazepine, fenobarbital en fenytoine niet combineren met itraconazol (Trisporal ${ }^{\oplus}$ ). Nederlands Tijdschrift voor Epileptologie 1999;27(4):135-6

Bootsma HPR, Toediening van anti-epileptica via de sonde. Nederlands Tijdschrift voor Epileptologie 2000;28(4):176-7

Hendriks H, Bootsma HPR, de Weerdt CJ Moederkruid getest voor profylaxe van migraine. Fytochemische en klinische aspecten van Tanacetum Parthenium. Pharm. Weekblad 2001;136(12):424-9

Aldenkamp AP, Arends J, Bootsma HPR, Diepman L, Hulsman J, Lambrechts D et al Randomised double-blind parallel-group study comparing cognitive effects of lamotrigine with valproate and placebo in healthy volunteers. Epilepsia 2002;43(1):19-26

Bootsma HPR, Anti-epileptic drugs and co-medication: suggestions for clinical practice. Acta Neurol. Belg. 2002(3):144 Abstract

Bootsma H, Coolen F, Leenen L, Lambrechts D, Hulsman J, Majoie M, Diepman L, Schellekens A, Arends J, Aldenkamp B Topiramate in an epilepsy centre; drug use evaluation study Epilepsia 2002;43(Suppl 8):121 Abstract

Bootsma HP, Coolen F, Aldenkamp AP, Arends J, Hulsman J, Lambrechts D, Leenen L, Majoie M, Schellekens A, de Krom M Topiramate in clinical practice: long term experience of patients with refractory epilepsy referred to a tertiary epilepsy centre. Epilepsia 2003;44(S8):117-8

Position paper radiofarmacie (mede-auteur) NVZA 2003

Bootsma HPR, Anti-epileptica en co-medicatie; handreikingen voor de praktijk. Tijdschrift voor Neurologie\&Neurochirurgie 2004;105(1):40-46

Bootsma HPR, Coolen F, Aldenkamp AP et al Topiramate in clinical practice: longterm experience in patients with refractory epilepsy referred to a tertiary epilepsy center. Epilepsy Behav. 2004;5(3):380-7

Roberts GM, Majoie MJH, Leenen LAM, Bootsma HP, Kessels FAGH, Aldenkamp AP, Leonard BE Ketter's hypothesis of the mood effects of anti-epileptic drugs coupled to the mechanism of action of topiramate and levetiracetam. Epilepsy Behav. 2005;6:366-72 
Aldenkamp AP and Bootsma HP Cognitive side-effects of antiepileptic drugs. In: Progress in Epileptic Disorders; Cognitive dysfunction in patients with temporal lobe epilepsy. Arzimanoglou A et al (Eds): John Libbey, Esher, Surrey,UK, pp. 217-233

Bootsma HP, Aldenkamp AP, Diepman L, Hulsman J, Lambrechts D, Leenen L, Majoie M, Schellekens A, Krom M de The effect of antiepileptic drugs on cognition: patient perceived cognitive problems of topiramate versus levetiracetam in clinical practice. Epilepsia 2006;47 Suppl 2:24-7

Bootsma HP, Ricker L, Diepman L, Gehring J, Hulsman J, Lambrechts D, Leenen L, Majoie M, Schellekens A, Krom M de, Aldenkamp AP Levetiracetam in clinical practice: long-term experience in patients with refractory epilepsy referred to a tertiary epilepsy center. Epilepsy Behav. 2007;10:296-303

Bootsma HP, Vos AM, Hulsman J, Lambrechts D, Majoie M, Schellekens A, Aldenkamp AP Lamotrigine in clinical practice: long-term experience in patients with refractory epilepsy referred to a tertiary epilepsy centre Epilepsy Behav. $2008 ; 12: 262-8$

Bootsma HP, Ricker L, Diepman L, Gehring J, Hulsman J, Lambrechts D, Leenen L, Majoie M, Schellekens A, de Krom M, Aldenkamp AP Long-term effects of levetiracetam and topiramate in clinical practice: A head-to-head comparison. Seizure 2008;17:19-26

Bootsma HP, Ricker L, Hulsman J, Lambrechts D, Majoie M, Schellekens A, Krom $\mathrm{M}$ de, Aldenkamp AP The impact of side effects on long-term retention in three new anti-epileptic drugs. Accepted for publication in Seizure 